\title{
On the separation of variables for the modular XXZ magnet and the lattice Sinh-Gordon models
}

\author{
Sergey É. Derkachov ${ }^{1}$ \\ Saint-Petersburg Department of Steklov Mathematical Institute of Russian Academy of Sciences, Fontanka 27, \\ 191023 St. Petersburg, Russia. \\ Karol K. Kozlowski ${ }^{2}$ \\ Univ Lyon, ENS de Lyon, Univ Claude Bernard Lyon 1, CNRS, Laboratoire de Physique, F-69342 Lyon, France. \\ Alexander N. Manashov ${ }^{3}$ \\ Institut für Theoretische Physik, Universität Hamburg, D-22761 Hamburg, Germany. \\ Institute for Theoretical Physics, University of Regensburg, D-93040 Regensburg, Germany. \\ Saint-Petersburg Department of Steklov Mathematical Institute of Russian Academy of Sciences, Fontanka 27, \\ 191023 St. Petersburg, Russia.
}

\begin{abstract}
We construct the generalised Eigenfunctions of the entries of the monodromy matrix of the $N$-site modular XXZ magnet and show, in each case, that these form a complete orthogonal system in $L^{2}\left(\mathbb{R}^{N}\right)$. In particular, we develop a new and simple technique, allowing one to prove the completeness of such systems. As a corollary of out analysis, we prove the Bystko-Teschner conjecture relative to the structure of the spectrum of the $\mathrm{B}(\lambda)$-operator for the odd length lattice Sinh-Gordon model.
\end{abstract}

\section{Introduction}

The quantum separation of variables has been developed by Sklyanin in [24, 25, 26] and was applied, since then, to obtain the spectra and Eigenstates of numerous quantum integrable models having finite and infinite dimensional local spaces $[3,7,8,16,17,21,20]$. The very essence of the method consists in mapping the original Hilbert

\footnotetext{
${ }^{1}$ e-mail: derkach@pdmi.ras.ru

${ }^{2}$ e-mail: karol.kozlowski@ens-lyon.fr

${ }^{3}$ e-mail: alexander.manashov@ desy.de
} 
space $\mathfrak{h}_{\text {org }}$ where a quantum integrable model is formulated onto an auxiliary Hilbert space $\mathfrak{h}_{\text {aux }}$. This mapping is done in a very specific way that strongly simplifies the original spectral problem for the model's transfer matrix. When formulated on the level of the original Hilbert space $\mathfrak{h}_{\text {org }}$, the spectral problem for the transfer matrix is a genuine multi-dimensional and multi-parameter spectral problem. However, upon implementing the separation of variables transform, the latter is re-expressed in terms of a multi-parameter but one-dimensional spectral problem which takes the form of a scalar $T-Q$ equation. Thus, the method provides an outstanding simplification.

An important ingredient of the whole construction consists in establishing that the mapping $\mathfrak{h}_{\text {org }} \rightarrow \mathfrak{h}_{\text {aux }}$ is unitary, a necessary step for guaranteeing the equivalence between the two Hilbert space descriptions of the spectral problem. In the finite dimensional setting, one may establish this unitarity by an direct counting of dimension arguments. However, the situation is disproportionnally harder in the case of integrable models associated with infinite dimensional local Hilbert spaces. Indeed, even in the simplest possible case, the quantum $N$-particle Toda chain [13], establishing the unitarity of the transform is equivalent to proving the completeness and orthogonality of the system of Whittaker functions for $\mathfrak{g l}_{N}$. This topic has a very long history. The satisfactory and full resolution of the problem was first achieved through rather evolved tools of harmonic analysis on non-compact groups [27]. These techniques, on top of their complexity, were completely unrelated to the algebraic structures usually dealt with in quantum integrable models. Hence their generalisation to more complex models was hardly imaginable. A first important progress towards a quantum integrability based proof of unitarity was achieved in [7]. That paper proposed a method for constructing a pyramidal -the so called Gauss-Givental[12] - representation for the integral kernel of the separation of variables transform in the case of the non-compact XXX sI $(2, \mathbb{C})$ chain. In fact, the integral kernel appeared as a generalised system of Eigenfunctions of one of the entries of the model's monodromy matrix. The work [7] developed a technique allowing one to establish orthogonality of this system, which is "half" of the proof of unitarity. The mentioned technique was later applied to other models subordinate to the rational $R$-matrix: the non-compact $\mathrm{XXX} \mathfrak{s l}(2, \mathbb{R})[8]$ or to the Toda chain [23]. A quantum inverse scattering based method for proving completeness was developed in [19], where all handlings leading to the proof of unitarity were set in a rigorous framework of distribution theory. Finally, one should mention the work [9] where, on the example of the $\mathfrak{s l}(2, \mathbb{C})$ chain, the technique of iterative construction of Eigenfunctions was perfected and set in a very natural to the quantum inverse scatterin method picture.

The purpose of the present paper is to push these developments further. On the one hand, we extend the method of constructing Gauss-Givental representations to the case of a non-compact, rank 1, model associated with a trigonometric $R$-matrix. One the other hand, we propose a simple method for proving completeness. Indeed, as observed in [19], Mellin-Barnes integral representations are well-suited for proving completeness of the unitarity transform's kernel. On a technical level, doing so demands to have at one's disposal a certain integral representation for the symmetric delta function in $N$-variables. A technique for proving this integral representation was developed in [19] and was based on a fine analysis of the consequences of the orthogonality relations. While providing the desired results, this method is not based on a direct argument. In the present paper, we develop a direct and systematic technique for establishing the integral identities of interest. We believe this to be an important contribution of our work. In this work, we focus our study on the case of the modular $X X Z$ magnet. This model is closely connected with the lattice Sinh-Gordon model. Thus, as a byproduct of our analysis, we prove the Bytsko-Teschner conjecture [3] relative to the structure of the spectrum of the B-operator in this model. Finally, at the time where this manuscript was beeing finalised, the work [22] appeared on the ArXiV and dealt, by slightly different means with a related problem in the context of the $q$-Toda chain. It would be interesting to compare the methods.

The paper is organised as follows. In Section 2 we introduce the modular XXZ chain's Lax matrix and discuss some of the fundamental objects related with this model. The Gauss-Givental integral representation for the system of Eigenfunctions associated with entries of the monodromy matrix is constructed in Section 3. It immediately allows us to establish the orthogonality of such a system. Section 4 is devoted to the proof of the 
completeness of the Eigensystems. We derive the Mellin-Barnes integral representation for the Eigenfunctions and evaluate some auxiliary integrals. Using these results we prove the completeness of the orthogonal systems associated with entries of the monodromy matrix. Then, in Section 5, we discuss properties of the complete orthogonal system associated with entries of the monodromy matrix. Finally, in Section 6, we apply our results to the case of the lattice Sinh-Gordon model. The paper contains two appendices. Appendix A reviews the various notations introduced in the paper. Appendix B reviews the special functions used throughout the work.

\section{The modular XXZ magnet}

\subsection{The model}

The modular XXZ magnet's Hilbert space has the tensor product decomposition $\mathfrak{h}=\otimes_{n=1}^{N} \mathfrak{h}_{n}$ where the Hilbert space $\mathfrak{h}_{n}$ associated with the $n^{\text {th }}$ site of the model is isomorphic to $L^{2}(\mathbb{R}, \mathrm{d} x)$. The $n^{\text {th }}$-site Lax matrix takes the form

$$
\mathrm{L}_{n}^{(\kappa)}(\lambda)=2\left(\begin{array}{cc}
-\mathrm{i} \sinh \left[\frac{\pi}{\omega_{1}}\left(\lambda-\mathrm{p}_{n}\right)\right] & \mathrm{e}^{-\pi \omega_{2} \mathrm{x}_{n}} \cosh \left[\frac{\pi}{\omega_{1}}\left(\mathrm{p}_{n}+\kappa\right)\right] \mathrm{e}^{-\pi \omega_{2} \mathrm{x}_{n}} \\
\mathrm{e}^{\pi \omega_{2} \mathrm{x}_{n}} \cosh \left[\frac{\pi}{\omega_{1}}\left(\mathrm{p}_{n}-\kappa\right)\right] \mathrm{e}^{\pi \omega_{2} \mathrm{x}_{n}} & -\mathrm{i} \sinh \left[\frac{\pi}{\omega_{1}}\left(\lambda+\mathrm{p}_{n}\right)\right]
\end{array}\right)
$$

Here and in the following, $\omega_{1}, \omega_{2}$ and $\kappa$ are three real parameters such that $\omega_{a}>0$ and $\kappa \neq 0$. Further, $\mathrm{x}_{n}$ and $\mathrm{p}_{n}$ are operators on $\mathfrak{h}_{n}$ satisfying to the canonical commutation relations $\left[\mathrm{x}_{n}, \mathrm{p}_{m}\right]=\delta_{n, m} \frac{\mathrm{i}}{2 \pi}$, where $\delta_{n, m}$ is the Kronecker symbol. In the following, we shall work in a representation where $\mathrm{x}_{n}$ is the multiplication operator by the $n^{\text {th }}$ coordinate

$$
\left(\mathrm{x}_{n} f\right)\left(\boldsymbol{x}_{N}\right)=x_{n} f\left(\boldsymbol{x}_{N}\right) \quad \text { with } \quad \boldsymbol{x}_{N}=\left(x_{1}, \ldots, x_{N}\right) \quad \text { so that } \quad\left(\mathrm{p}_{n} f\right)\left(\boldsymbol{x}_{N}\right)=-\frac{\mathrm{i}}{2 \pi} \partial_{x_{n}} f\left(\boldsymbol{x}_{N}\right) .
$$

The model's monodromy matrix takes the form

$$
\mathrm{T}_{N}(\lambda)=\mathrm{L}_{1}^{(\kappa)}(\lambda) \cdots \mathrm{L}_{N}^{(\kappa)}(\lambda)=\left(\begin{array}{ll}
\mathrm{A}_{N}(\lambda) & \mathrm{B}_{N}(\lambda) \\
\mathrm{C}_{N}(\lambda) & \mathrm{D}_{N}(\lambda)
\end{array}\right)
$$

The transfer matrix

$$
\mathrm{t}(\lambda)=\operatorname{tr}[\mathrm{T}(\lambda)]=(-\mathrm{i})^{N} \mathrm{e}^{\frac{\pi}{\omega_{1}} N \lambda} \sum_{k=0}^{N}\left(-\mathrm{e}^{-\frac{2 \pi}{\omega_{1}} \lambda}\right)^{k} \mathrm{~T}_{k}
$$

provides ones with a commutative algebra of positive self-adjoint operators $\left\{\mathrm{T}_{k}\right\}_{1}^{N}$ on $\mathfrak{h}$, c.f. [3] for more details.

\subsection{The elementary operator relations of the model}

The $\mathfrak{R}$-operator for the modular XXZ magnet was first constructed in [2]. Its non-trivial part was given as a special function of a positive self-adjoint operator coocked up from a representation of the coproduct of the Casimir of $U_{q}\left(\mathfrak{s l}_{2}\right)$. That charasterisation of $\mathfrak{R}$ was rather implicit. The paper [4] proposed an alternative way to construct this $\Re$-operator. This allowed for a much simpler and more explicit form of $\Re$. This construction was based on the existence of an alternative factorisation of the model's Lax matrix

$$
\mathrm{L}_{n}^{(\kappa)}(\lambda)=-\mathrm{i} \mathrm{M}_{u_{2}}\left(\mathrm{x}_{n}\right) \cdot \mathrm{H}\left(\mathrm{p}_{n}\right) \cdot \mathrm{N}_{u_{1}}\left(\mathrm{x}_{n}\right)
$$


where

$$
\mathrm{M}_{u_{2}}(\mathrm{x})=\left(\begin{array}{cc}
U_{2} & -U_{2}^{-1} \\
-U_{2}^{-1} \mathrm{e}^{2 \pi \omega_{2} \mathrm{x}} & U_{2} \mathrm{e}^{2 \pi \omega_{2} \mathrm{x}}
\end{array}\right), \quad \mathrm{N}_{u_{1}}(\mathrm{x})=\left(\begin{array}{cc}
-U_{1} & U_{1}^{-1} \mathrm{e}^{-2 \pi \omega_{2} \mathrm{x}} \\
-U_{1}^{-1} & U_{1} \mathrm{e}^{-2 \pi \omega_{2} \mathrm{x}}
\end{array}\right)
$$

and $\mathrm{H}(\mathrm{p})=\mathrm{e}^{-\frac{\pi}{\omega_{1}}\left(\mathrm{p}-\mathrm{i} \frac{\Omega}{2}\right) \sigma_{3}}$. Here, $\sigma_{3}=\operatorname{diag}(1,-1)$ and we made use of the notation

$$
U_{a}=\mathrm{e}^{\frac{\pi}{\omega_{1}} u_{a}} \quad \text { with } \quad\left\{\begin{array}{l}
u_{1}=\frac{1}{2} \cdot\left(\lambda+\kappa-\mathrm{i} \frac{\tau}{2}\right) \\
u_{2}=\frac{1}{2} \cdot\left(\lambda-\kappa-\mathrm{i} \frac{\tau}{2}\right)
\end{array},\right.
$$

and, agree, from now on, to denote

$$
\Omega=\omega_{1}+\omega_{2}, \quad \tau=\omega_{2}-\omega_{1} .
$$

The factorisation (2.5) allows one to interpret the Lax matrix as a function of the parameters $u_{1}$ and $u_{2}$, viz.

$$
\mathrm{L}_{n}^{(\kappa)}(\lambda) \equiv \mathrm{L}_{n}\left(u_{1}, u_{2}\right)
$$

We shall adopt this notation in the following. Note that changing $\kappa \hookrightarrow-\kappa$ produces and exchange of the parameters $u_{1}$ and $u_{2}$. It is well known that the operator $D_{-\kappa}(\mathrm{p})$, the function $D_{\alpha}$ being defined in Appendix B c.f. (B.8), is an intertwining operator between the $\kappa$ and $-\kappa$ representations meaning that

$$
D_{u_{2}-u_{1}}\left(\mathrm{p}_{1}\right) \cdot \mathrm{L}_{1}\left(u_{1}, u_{2}\right)=\mathrm{L}_{1}\left(u_{2}, u_{1}\right) \cdot D_{u_{2}-u_{1}}\left(\mathrm{p}_{1}\right) .
$$

The operator $D_{\alpha}(\mathrm{p})$ acts as a multiplication operator on the spectrum of $\mathrm{p}_{1}$ and can be represented as an integral operator on the spectrum of $\mathrm{x}_{1}$ by means of the Fourier transform (B.16):

$$
\left[D_{\alpha}(\mathrm{p}) \cdot f\right](x)=\sqrt{\omega_{1} \omega_{2}} \mathcal{A}(\alpha) \int_{\mathbb{R}} D_{\alpha^{\star}}\left(\omega_{1} \omega_{2}\left(x-x^{\prime}\right)\right) f\left(x^{\prime}\right) \cdot \mathrm{d} x^{\prime}
$$

where we have introduced

$$
\alpha^{\star}=-\alpha-\mathrm{i} \frac{\Omega}{2} \quad \text { and } \quad \mathcal{A}(\alpha)=\varpi\left(\alpha-\alpha^{\star}\right)=\varpi\left(2 \alpha+\mathrm{i} \frac{\Omega}{2}\right) .
$$

Also, $\varpi$ refers to the quantum dilogarithm whose definition is recalled in Appendix B c.f. (B.1).

It was established in [4], on the basis of the factorisation (2.5), that it holds

$$
D_{u_{1}-v_{2}}\left(\omega_{1} \omega_{2} \mathrm{x}_{12}\right) \mathrm{L}_{1}\left(u_{1}, u_{2}\right) \mathrm{L}_{2}\left(v_{1}, v_{2}\right)=\mathrm{L}_{1}\left(v_{2}, u_{2}\right) \mathrm{L}_{2}\left(v_{1}, u_{1}\right) D_{u_{1}-v_{2}}\left(\omega_{1} \omega_{2} \mathrm{x}_{12}\right)
$$

Above and in the following, for any quantities $\alpha_{a}, \alpha_{b}$ we agree to denote $\alpha_{a b}=\alpha_{a}-\alpha_{b}$, e.g. $\mathrm{x}_{12}=\mathrm{x}_{1}-\mathrm{x}_{2}$.

The two above intertwining relations thus ensure that the operator

$$
\begin{aligned}
\mathrm{R}_{12}\left(u_{1}, u_{2} \mid v_{2}\right) & =D_{u_{2}-u_{1}}\left(\omega_{1} \omega_{2} \mathrm{x}_{12}\right) \cdot D_{u_{2}-v_{2}}\left(\mathrm{p}_{1}\right) \cdot D_{u_{1}-v_{2}}\left(\omega_{1} \omega_{2} \mathrm{x}_{12}\right) \\
& =D_{u_{1}-v_{2}}\left(\mathrm{p}_{1}\right) \cdot D_{u_{2}-v_{2}}\left(\omega_{1} \omega_{2} \mathrm{x}_{12}\right) \cdot D_{u_{2}-u_{1}}\left(\mathrm{p}_{1}\right)
\end{aligned}
$$

realises the intertwining

$$
\mathrm{R}_{12}\left(u_{1}, u_{2} \mid v_{2}\right) \mathrm{L}_{1}\left(u_{1}, u_{2}\right) \mathrm{L}_{2}\left(v_{1}, v_{2}\right)=\mathrm{L}_{1}\left(u_{1}, v_{2}\right) \mathrm{L}_{2}\left(v_{1}, u_{2}\right) \mathrm{R}_{12}\left(u_{1}, u_{2} \mid v_{2}\right) .
$$


The two factorisations of $\mathrm{R}_{12}$ stem from the two possible ways of decomposing the permutation

$$
\left(u_{1}, u_{2}, v_{1}, v_{2}\right) \mapsto\left(u_{1}, v_{2}, v_{1}, u_{2}\right)
$$

into 2-cycles. Note that the equality between the two factorisations is, in fact, a consequence of the three term integral relation (B.20) satisfied by the $D$ functions which, in an operator form, is given in (B.24). Also, we stress that $\mathrm{R}_{12}$ does not correspond to the $\Re$ operator of the XXZ-modular magnet. Nonetheless, it corresponds to half of its building block. We refer to [4] for more details.

Finally, observe that one has the identities

$$
\boldsymbol{v}_{\epsilon}^{t} \cdot \mathrm{L}_{n}\left(u_{1}, u_{2}\right) \cdot \mathrm{e}^{-2 \mathrm{i} \pi \epsilon\left(v_{2}-u_{2}\right) \mathrm{x}_{n}}=\mathrm{e}^{-2 \mathrm{i} \pi \epsilon\left(v_{2}-u_{2}\right) \mathrm{x}_{n}} \cdot \boldsymbol{v}_{\epsilon}^{t} \cdot \mathrm{L}_{n}\left(u_{1}, v_{2}\right)
$$

and

$$
\mathrm{L}_{n}\left(u_{1}, v_{2}\right) \boldsymbol{v}_{\epsilon} \cdot \mathrm{e}^{2 \mathrm{i} \pi \epsilon\left(u_{1}-v_{1}\right) \mathrm{x}_{n}}=\mathrm{e}^{2 \mathrm{i} \pi \epsilon\left(u_{1}-v_{1}\right) \mathrm{x}_{n}} \cdot \mathrm{L}_{n}\left(v_{1}, v_{2}\right) \boldsymbol{v}_{\epsilon}
$$

where we have introduced

$$
\boldsymbol{v}_{+}=\left(\begin{array}{l}
1 \\
0
\end{array}\right) \quad \text { and } \quad \boldsymbol{v}_{-}=\left(\begin{array}{l}
0 \\
1
\end{array}\right) .
$$

\section{The Gauss-Givental integral representation and orthogonality}

\subsection{The $\Lambda$-operator}

We have now introduced enough notations so as to introduce the $\Lambda_{y}^{(N)}$ operators and their adjoints. These operators play a crucial role in constructing the generalised Eigenfunctions of the operators $\mathrm{A}_{N}$ and $\mathrm{B}_{N}$. Below, we shall establish some of their exchange relations which are important for our further purposes. These should be understood in the weak sense, and when one considers sufficiently regular functions. See [19] for more details.

Definition 3.1 Let $y_{ \pm}$stand for

$$
y_{ \pm}=\frac{1}{2}\left(y \pm \kappa-\mathrm{i} \frac{\Omega}{2}\right) \text {. }
$$

Denote by $\Lambda_{y, \epsilon}^{(N)}$ the below continuous operators $L^{\infty}\left(\mathbb{R}^{N-1}\right) \mapsto L^{\infty}\left(\mathbb{R}^{N}\right)$ :

$$
\begin{aligned}
\Lambda_{y, \epsilon}^{(N)} & =\frac{\mathrm{e}^{2 \mathrm{i} \pi y_{-} x_{1}}}{\left(\mathcal{A}\left(y_{+}\right) \sqrt{\omega_{1} \omega_{2}}\right)^{N-1}} \cdot \widetilde{\jmath}_{N}\left(y_{-}-y_{+}\right) \cdot U_{N}\left(y_{-}, y_{+}\right) \cdot \mathrm{e}^{2 \mathrm{i} \pi \epsilon y_{+}^{\star} x_{N}} 1_{N} \\
& =\frac{\mathrm{e}^{2 \mathrm{i} \pi y_{-} x_{1}}}{\left(\mathcal{A}\left(y_{+}\right) \sqrt{\omega_{1} \omega_{2}}\right)^{N-1}} \cdot U_{N}\left(y_{+}, y_{-}\right) \cdot J_{N}\left(y_{-}-y_{+}\right) \cdot \mathrm{e}^{2 \mathrm{i} \pi \epsilon y_{+}^{\star} x_{N}} 1_{N}
\end{aligned}
$$

with

$$
\widetilde{J}_{N}(y)=\prod_{a=1}^{N-1} D_{y}\left(\omega_{1} \omega_{2} x_{a a+1}\right), \quad J_{N}(y)=\prod_{a=1}^{N-1} D_{y}\left(p_{a}\right)
$$

as well as

$$
U_{N}\left(y_{+}, y_{-}\right)=\prod_{a}^{1 \curvearrowright N-1}\left\{D_{y_{+}}\left(p_{a}\right) D_{y_{-}}\left(\omega_{1} \omega_{2} x_{a a+1}\right)\right\} .
$$

Finally, the operator $1_{N}$ stands for the constant function on the $N^{\text {th }}$ space and the formula is to be understood as the partial action of the chain of operators on this function. 
Above and in the following we agree to denote the ordered products as

$$
\prod_{a}^{1 \curvearrowright N} 0_{a}=0_{1} \cdots 0_{N} \quad \text { and } \quad \prod_{a}^{N \curvearrowright 1} 0_{a}=0_{N} \cdots 0_{1} .
$$

Furthermore, the equality between (3.2) and (3.3) follows from a multiple application of the relation (B.24).

Owing to the conjugation property of the $D_{\alpha}$ function $c . f$. (B.8), and those of the quantum dilogarithm, the adjoint operators

$$
\begin{aligned}
\left(\Lambda_{y, \epsilon}^{(N)}\right)^{\dagger} & =\left(\frac{\mathcal{A}\left(\left(-y_{+}^{*}\right)^{\star}\right)}{\sqrt{\omega_{1} \omega_{2}}}\right)^{N-1} \cdot 1_{N}^{\dagger} \mathrm{e}^{-2 \mathrm{i} \pi \epsilon\left(y_{+}^{\star}\right)^{*} \mathrm{x}_{N}} \mathrm{U}_{N}^{\dagger}\left(y_{-}, y_{+}\right) \widetilde{\mathrm{J}}_{N}^{-1}\left(y_{-}-y_{+}\right) \mathrm{e}^{-2 \mathrm{i} \pi y_{-}^{*} \mathrm{x}_{1}} \\
& =\left(\frac{\mathcal{A}\left(\left(-y_{+}^{*}\right)^{\star}\right)}{\sqrt{\omega_{1} \omega_{2}}}\right)^{N-1} \cdot 1_{N}^{\dagger} \mathrm{e}^{-2 \mathrm{i} \pi \epsilon\left(y_{+}^{\star}\right)^{*} \mathrm{x}_{N}} \mathrm{~J}_{N}^{-1}\left(y_{-}-y_{+}\right) \mathrm{U}_{N}^{\dagger}\left(y_{+}, y_{-}\right) \mathrm{e}^{-2 \mathrm{i} \pi y_{-}^{*} \mathrm{x}_{1}}
\end{aligned}
$$

give rise to continuous operators $L^{\infty}\left(\mathbb{R}^{N}\right) \rightarrow L^{\infty}\left(\mathbb{R}^{N-1}\right)$. Here, * stands for the complex conjugation while ${ }^{\star}$ for the transform (2.12). Moreover, $1_{N}^{\dagger}$ represents the operation of integration versus the $N^{\text {th }}$ coordinate. Finally, we have set

$$
\mathrm{U}_{N}^{\dagger}\left(y_{+}, y_{-}\right)=\prod_{a}^{N-1 \curvearrowright 1} D_{-y_{-}^{*}}\left(\omega_{1} \omega_{2} \mathbf{x}_{a a+1}\right) D_{-y_{+}^{*}}\left(\mathrm{p}_{a}\right)
$$

We stress that the $\Lambda$-operators are in fact very closely related to the partial R-operators in that they can be expressed as

$$
\Lambda_{y, \epsilon}^{(N)}=\frac{\mathrm{e}^{2 \mathrm{i} \pi y_{-} \mathrm{x}_{1}}}{\left(\mathcal{A}\left(y_{+}\right) \sqrt{\omega_{1} \omega_{2}}\right)^{N-1}} \cdot \mathrm{R}_{12}\left(u_{1}, u_{2} \mid v_{2}\right) \cdots \mathrm{R}_{N-1 N}\left(u_{1}, u_{2} \mid v_{2}\right) \cdot \mathrm{e}^{2 \mathrm{i} \pi \epsilon y_{+}^{\star} \mathrm{x}_{N}},
$$

where $y_{ \pm}$are as defined in (3.1).

The first interesting property of the operators $\Lambda_{y, \pm}^{(N)}$ concerns their exchange relations with the operators $\mathrm{A}_{N}, \mathrm{~B}_{N}$.

Lemma 3.1 The operators $\Lambda_{y, \epsilon}^{(N)}$ satisfy

$$
\begin{aligned}
& A_{N}(\lambda) \cdot \Lambda_{y,-}^{(N)}=-2 \mathrm{i} \sinh \left[\frac{\pi}{\omega_{1}}(\lambda-y)\right] \cdot \Lambda_{y,-}^{(N)} \cdot A_{N-1}(\lambda) \\
& B_{N}(\lambda) \cdot \Lambda_{y,+}^{(N)}=-2 \mathrm{i} \sinh \left[\frac{\pi}{\omega_{1}}(\lambda-y)\right] \cdot \Lambda_{y,+}^{(N)} \cdot B_{N-1}(\lambda) .
\end{aligned}
$$

\section{Proof -}

Introduce the notation

$$
\mathrm{T}_{N}\left(u_{1}, u_{2}\right) \equiv \mathrm{T}_{N}(\lambda)=\mathrm{L}_{1}\left(u_{1}, u_{2}\right) \cdot \mathrm{L}_{2}\left(u_{1}, u_{2}\right) \cdots \mathrm{L}_{N}\left(u_{1}, u_{2}\right),
$$

where $u_{a}$ 's are given as in (2.7). Then, by virtue of (2.16), (2.18), (2.19), for arbitrary $v_{1}, v_{2}$, the operators

$$
0_{\epsilon^{\prime}, \epsilon}^{(N)}\left(u_{1}, u_{2} \mid v_{1}, v_{2}\right)=\mathrm{e}^{-2 \mathrm{i} \pi \epsilon^{\prime}\left(v_{2}-u_{2}\right) \mathrm{x}_{1}} \cdot \mathrm{R}_{12}\left(u_{1}, u_{2} \mid v_{2}\right) \cdots \mathrm{R}_{N-1 N}\left(u_{1}, u_{2} \mid v_{2}\right) \cdot \mathrm{e}^{2 \mathrm{i} \pi \epsilon\left(u_{1}-v_{1}\right) \mathrm{x}_{N}}
$$

satisfy to the exchange relation

$$
\boldsymbol{v}_{\epsilon^{\prime}}^{t} \cdot \mathrm{T}_{N}\left(u_{1}, u_{2}\right) \boldsymbol{v}_{\epsilon} \cdot 0_{\epsilon^{\prime}, \epsilon}^{(N)}\left(u_{1}, u_{2} \mid v_{2}\right)=0_{\epsilon^{\prime}, \epsilon}^{(N)}\left(u_{1}, u_{2} \mid v_{1}, v_{2}\right) \cdot \boldsymbol{v}_{\epsilon^{\prime}}^{t} \cdot \mathrm{T}_{N-1}\left(u_{1}, u_{2}\right) \cdot \mathrm{L}_{N}\left(v_{1}, v_{2}\right) \boldsymbol{v}_{\epsilon} .
$$


Upon adopting the parameterisation of $v_{1}, v_{2}$ in the form

$$
v_{1}=\frac{1}{2}\left(\mu+s-\mathrm{i} \frac{\tau}{2}\right) \quad \text { and } \quad v_{2}=\frac{1}{2}\left(\mu-s-\mathrm{i} \frac{\tau}{2}\right),
$$

agreeing upon $\chi_{N ; \epsilon}\left(x_{N}\right)=\mathrm{e}^{2 \mathrm{i} \pi \epsilon x_{N}\left(s+\mathrm{i} \frac{\Omega}{2}\right)}$, it is readily checked that

$$
\begin{aligned}
\left(v_{+}^{t} \mathrm{~T}_{N-1}\left(u_{1}, u_{2}\right) \cdot \mathrm{L}_{N}\left(v_{1}, v_{2}\right)\right. & \left.\boldsymbol{v}_{\epsilon} \cdot \chi_{N ; \epsilon}\right)\left(x_{N}\right) \\
& =-2 \mathrm{i} \sinh \left[\frac{\pi}{\omega_{1}}\left(\mu-s-\mathrm{i} \frac{\Omega}{2}\right)\right]\left(\mathrm{A}_{N-1}(\lambda) \delta_{\epsilon, 1}+\mathrm{B}_{N-1}(\lambda) \delta_{\epsilon,-1}\right) \cdot \chi_{N ; \epsilon}\left(x_{N}\right) .
\end{aligned}
$$

Finally, consider the re-parametrisation

$$
v_{2}=\frac{1}{2}\left(\lambda-y+\mathrm{i} \omega_{1}\right) \quad \text { and } \quad v_{1}=v_{2}+s \quad \text { so that } \quad\left\{\begin{array}{c}
v_{2}-u_{2}=-y_{-} \\
u_{1}-v_{1}+s+\mathrm{i} \frac{\Omega}{2}=-y_{+}^{\star}
\end{array}\right.
$$

where $y_{ \pm}$are as defined in (3.1). These then lead to the rewriting

$$
\begin{array}{r}
\mathrm{R}_{12}\left(u_{1}, u_{2} \mid v_{2}\right)=D_{y_{-}-y_{+}}\left(\omega_{1} \omega_{2} \mathrm{x}_{12}\right) \cdot D_{y_{-}}\left(\mathrm{p}_{1}\right) \cdot D_{y_{+}}\left(\omega_{1} \omega_{2} \mathrm{x}_{12}\right) \\
=D_{y_{+}}\left(\mathrm{p}_{1}\right) \cdot D_{y_{-}}\left(\omega_{1} \omega_{2} \mathrm{x}_{12}\right) \cdot D_{y_{-}-y_{+}}\left(\mathrm{p}_{1}\right),
\end{array}
$$

which entails

$$
0_{+, \epsilon}^{(N)}\left(u_{1}, u_{2} \mid v_{1}, v_{2}\right) \cdot \chi_{N ; \epsilon}=\left(\mathcal{A}\left(y_{+}\right) \sqrt{\omega_{1} \omega_{2}}\right)^{N-1} \cdot \Lambda_{y,-\epsilon}^{(N)} .
$$

All together with (3.15)-(3.17) this yields the two representations given in (3.11)-(3.12)

We now establish exchange relation between the $\Lambda_{y, \pm}^{(N)}$ operators on the one hand and, on the other hand, between these operators and their duals. Below, we shall adopt hypergeometric-like notations for product of functions, as defined in (A.3)

Proposition 3.2 Given $y, t \in \mathbb{R}$, let $y_{ \pm}, t_{ \pm}$be defined according to (3.1).

For $y \neq t$, the operators $\left(\Lambda_{y, \epsilon}^{(N)}\right)^{\dagger}$ and $\Lambda_{t, \epsilon}^{(N)}$ satisfy to the exchange relations

$$
\left(\Lambda_{y, \epsilon}^{(N)}\right)^{\dagger} \cdot \Lambda_{t, \epsilon}^{(N)}=\frac{1}{\omega_{1} \omega_{2}} \cdot \mathcal{A}\left(t_{+}^{\star}-\left(y_{+}^{\star}\right)^{*}, t_{-}-y_{-}^{*}\right) \Lambda_{t, \epsilon}^{(N-1)} \cdot\left(\Lambda_{y ; \epsilon}^{(N-1)}\right)^{\dagger}
$$

and enjoy the pseudo-commutation relations

$$
\Lambda_{y, \epsilon}^{(N)} \Lambda_{t, \epsilon}^{(N-1)}=\Lambda_{t, \epsilon}^{(N)} \Lambda_{y, \epsilon}^{(N-1)} .
$$

Proof-

In order to establish the pseudo-commutativity, observe that

$$
\Lambda_{y, \epsilon}^{(N)} \Lambda_{t, \epsilon}^{(N-1)}=\frac{\left(\mathcal{A}\left(t_{+}, y_{+}\right)\right)^{2-N}}{\left(\omega_{1} \omega_{2}\right)^{N-\frac{3}{2}}} \widetilde{\mathrm{J}}_{N}\left(y_{-}-y_{+}\right) \cdot \mathrm{P}(y, t) \cdot \mathrm{J}_{N-1}\left(t_{-}-t_{+}\right) \cdot 1_{N} \otimes 1_{N-1}
$$

where

$$
\mathrm{P}(y, t)=\mathcal{A}\left(t_{+}\right) \mathrm{e}^{2 \mathrm{i} \pi y_{-} \mathrm{x}_{1}} \cdot \mathrm{U}_{N}\left(y_{-}, y_{+}\right) \cdot \mathrm{e}^{2 \mathrm{i} \pi \epsilon y_{+}^{\star} \mathrm{x}_{N}} \cdot \mathrm{e}^{2 \mathrm{i} \pi t_{-} \mathrm{x}_{1}} \cdot \mathrm{U}_{N-1}\left(t_{+}, t_{-}\right) \cdot \mathrm{e}^{2 \mathrm{i} \pi \epsilon t_{+}^{\star} \mathrm{x}_{N-1}}
$$


Since $y_{+}-y_{-}=t_{+}-t_{-}$, it is enough to establish that $\mathrm{P}(y, t)$ is symmetric under the exchange $t \leftrightarrow y$.

One can recast $\mathrm{P}(y, t)$ in the below product form

$$
\begin{aligned}
\mathrm{P}\left(y_{ \pm}, t_{ \pm}\right)=\left[\mathrm{e}^{2 \mathrm{i} \pi y_{-} \mathrm{x}_{1}} D_{y_{-}}\left(\mathrm{p}_{1}\right) \cdot D_{y_{+}}\left(\omega_{1} \omega_{2} \mathrm{x}_{12}\right) \mathrm{e}^{2 \mathrm{i} \pi t_{-} \mathrm{x}_{1}} D_{t_{+}}\left(\mathrm{p}_{1}\right)\right] \\
\times \prod_{a}^{2 \curvearrowright N-2}\left\{D_{y_{-}}\left(\mathrm{p}_{a}\right) \cdot D_{y_{+}}\left(\omega_{1} \omega_{2} \mathrm{x}_{a a+1}\right) D_{t_{-}}\left(\omega_{1} \omega_{2} \mathrm{x}_{a a-1}\right) D_{t_{+}}\left(\mathrm{p}_{a}\right)\right\} \\
\quad \times \mathcal{A}\left(t_{+}\right)\left[D_{y_{-}}\left(\mathrm{p}_{N-1}\right) \cdot D_{y_{+}}\left(\omega_{1} \omega_{2} \mathrm{x}_{N-1 N}\right) D_{t_{-}}\left(\omega_{1} \omega_{2} \mathrm{x}_{N-2 N-1}\right) \mathrm{e}^{2 \mathrm{i} \pi \epsilon\left(t_{+}^{\star} \mathrm{x}_{N-1}+y_{+}^{\star} \mathrm{x}_{N}\right)}\right] .
\end{aligned}
$$

At this stage it remains to invoke the relations (B.26), (B.27) and (B.28) so as to conclude that $\mathrm{P}\left(y_{ \pm}, t_{ \pm}\right)$is symmetric in $t \leftrightarrow y$.

The exchange relation (3.21) for the adjoint can be established by means of the integral relations (B.23)-(B.22) for the $D_{\alpha}$ functions. Also, one should use that provided $f$ is a regular function, one has the integral representations

$$
\begin{aligned}
& {\left[\mathrm{e}^{2 \mathrm{i} \pi t_{-} \mathrm{x}_{1}} \cdot \mathrm{U}_{N}\left(t_{-}, t_{+}\right) \cdot \mathrm{e}^{2 \mathrm{i} \pi \epsilon t_{+}^{\star} \mathrm{x}_{N}} 1_{N} \cdot f\right]\left(\boldsymbol{x}_{N}^{\prime}\right)} \\
& =\int_{\mathbb{R}^{N-1}} \mathrm{e}^{2 \mathrm{i} \pi t_{-} x_{1}^{\prime}} \cdot \prod_{a=1}^{N-1}\left\{\tilde{\mathcal{A}}\left(t_{-}\right) \cdot D_{t_{-}^{\star}}\left(\omega_{1} \omega_{2}\left(x_{a}^{\prime}-z_{a}\right)\right) \cdot D_{t_{+}}\left(\omega_{1} \omega_{2}\left(x_{a+1}^{\prime}-z_{a}\right)\right)\right\} \cdot \mathrm{e}^{2 \mathrm{i} \pi \epsilon t_{+}^{\star} x_{N}^{\prime}} f\left(z_{N-1}\right) \cdot \mathrm{d}^{N-1} z
\end{aligned}
$$

where $\widetilde{\mathcal{A}}(\alpha)=\sqrt{\omega_{1} \omega_{2}} \mathcal{A}(\alpha)$. Analogously, one has

$$
\begin{aligned}
& {\left[1_{N}^{\dagger} \mathrm{e}^{-2 \mathrm{i} \pi \epsilon\left(y_{+}^{\star}\right)^{*} \mathrm{x}_{N}} \cdot \mathrm{U}_{N}^{\dagger}\left(y_{-}, y_{+}\right) \cdot \mathrm{e}^{-2 \mathrm{i} \pi \epsilon y_{-}^{*} \mathrm{x}_{1}} \cdot g\right]\left(\boldsymbol{x}_{N-1}^{\prime}\right)} \\
& =\int_{\mathbb{R}^{N}} \mathrm{e}^{-2 \mathrm{i} \pi \epsilon\left(y_{+}^{\star}\right)^{*} z_{N}} \cdot \prod_{a=1}^{N-1}\left\{\widetilde{\mathcal{A}}\left(-y_{-}^{*}\right) \cdot D_{\left(-y_{-}^{*}\right)^{\star}}\left(\omega_{1} \omega_{2}\left(x_{a}^{\prime}-z_{a}\right)\right) \cdot D_{-y_{+}^{*}}\left(\omega_{1} \omega_{2}\left(z_{a+1}-x_{a}^{\prime}\right)\right)\right\} \cdot \mathrm{e}^{-2 \mathrm{i} \pi y_{-}^{*} z_{1}} g\left(z_{N-1}\right) \cdot \mathrm{d}^{N} z
\end{aligned}
$$

Above, we denote by $\boldsymbol{x}_{k}$ the $k$-dimensional vector $\boldsymbol{x}_{k}=\left(x_{1}, \ldots, x_{k}\right)$. The claim then follows after a longish but straightforward calculation based on the integral identities (B.22), (B.23).

\subsection{Further properties of the $\Lambda$ operators}

\section{Proposition 3.3 It holds}

$$
B_{N}(y) \cdot \Lambda_{y,-}^{(N)}=\{b(y)\}^{N} \cdot \Lambda_{y+\mathrm{i} \omega_{2},-}^{(N)} \quad \text { with } \quad b(\lambda)=-2 \mathrm{i} \sinh \left[\frac{\pi}{\omega_{1}}\left(\lambda+\kappa+\mathrm{i} \frac{\Omega}{2}\right)\right]
$$

and

$$
C_{N}(y) \cdot \Lambda_{y,-}^{(N)}=\{c(y)\}^{N} \cdot \Lambda_{y-\mathrm{i} \omega_{2},-}^{(N)} \quad \text { with } \quad c(\lambda)=-2 \mathrm{i} \sinh \left[\frac{\pi}{\omega_{1}}\left(\lambda-\kappa-\mathrm{i} \frac{\Omega}{2}\right)\right] .
$$

Furthermore, dual relations holds for the dual objects.

One possible way to establish the above relations is based on the use of the gauge transformation initially suggested in the paper [10] for the derivation of the Baxter $T-Q$ equation in the case of the Toda chain and adapted to the case of XXX-spin chain in [6] and later used in [1, 7, 8, 9]. Here, however, but we shall present a proof which adapts, to the situation of interest, the reasoning introduced in [5]. Although we do not provide these 
here, analogous relations can be obtained within the same technique for the action of the $\mathrm{A}_{N}, \mathrm{D}_{N}$ operators on the $\Lambda_{y,+}^{(N)}$ operator.

Proof-

The intertwining relation (2.16) may be recast in the form

$$
\mathrm{R}_{12}\left(u_{1}, u_{2} \mid v_{2}\right) \mathrm{L}_{1}\left(u_{1}, u_{2}\right)=\mathrm{L}_{1}\left(u_{1}, v_{2}\right) \mathrm{L}_{2}\left(u_{1}, u_{2}\right) \mathrm{R}_{12}\left(u_{1}, u_{2} \mid v_{2}\right) \cdot \mathrm{L}_{2}^{-1}\left(u_{1}, v_{2}\right) .
$$

Then, by using the elementary decomposition of the L matrices (2.5), one gets the identity

$$
\mathrm{R}_{12}\left(u_{1}, u_{2} \mid v_{2}\right) \mathrm{L}_{1}\left(u_{1}, u_{2}\right)=-\mathrm{i} \mathrm{M}_{v_{2}}\left(\mathrm{x}_{1}\right) \cdot \mathrm{G} \cdot \mathrm{M}_{v_{2}}^{-1}\left(\mathrm{x}_{2}\right)
$$

with

$$
\mathrm{G}=\mathrm{H}\left(\mathrm{p}_{1}\right) \cdot \mathrm{N}_{u_{1}}\left(\mathrm{x}_{1}\right) \cdot \mathrm{M}_{u_{2}}\left(\mathrm{x}_{2}\right) \cdot \mathrm{H}\left(\mathrm{p}_{2}\right) \cdot \mathrm{R}_{12}\left(u_{1}, u_{2} \mid v_{2}\right) \cdot \mathrm{H}^{-1}\left(\mathrm{p}_{2}\right) .
$$

Upon multiplication by the gauge matrices

$$
\mathrm{Z}_{k}=\left(\begin{array}{cc}
1 & 0 \\
\mathrm{e}^{2 \pi \omega_{2} \mathrm{x}_{k}} & 1
\end{array}\right)
$$

the above relation takes the form

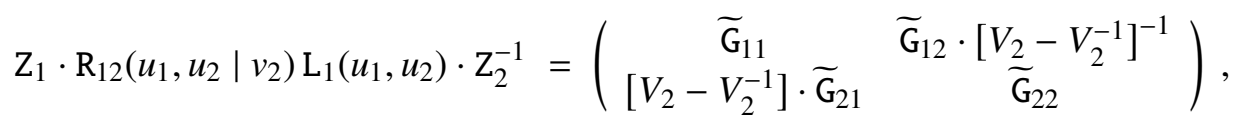

where $V_{2}$ is defined according to (2.7) and we have set

$$
\widetilde{G}=\frac{-\mathrm{i}}{V_{2}+V_{2}^{-1}}\left(\begin{array}{cc}
V_{2} & -V_{2}^{-1} \\
-V_{2}^{-1} \mathrm{e}^{2 \pi \omega_{2} \mathrm{x}_{1}} & V_{2} \mathrm{e}^{2 \pi \omega_{2} \mathrm{x}_{1}}
\end{array}\right) \cdot \mathrm{G} \cdot\left(\begin{array}{cc}
-V_{2}^{-1} \mathrm{e}^{-2 \pi \omega_{2} \mathrm{x}_{2}} \\
-1 & V_{2} \mathrm{e}^{-2 \pi \omega_{2} \mathrm{x}_{2}}
\end{array}\right) .
$$

The matrix elements of $\widetilde{G}$ are smooth in $V_{2}$ belonging to a vicinity of 1. Furthemore the lhs of (3.34) has a welldefinied limit when $v_{2} \rightarrow 0$. Thus, the 12-enrty of the rhs of (3.34) admits a $V_{2} \rightarrow 1$ limit. Furthermore, the 21-entry vanishes in this limit. Hence, one has the structure

$$
\mathrm{Z}_{1} \cdot \mathrm{R}_{12}\left(u_{1}, u_{2} \mid 0\right) \mathrm{L}_{1}\left(u_{1}, u_{2}\right) \cdot \mathrm{Z}_{2}^{-1}=\left(\begin{array}{cc}
\widetilde{\mathrm{G}}_{11} & * \\
0 & \widetilde{\mathrm{G}}_{22}
\end{array}\right)_{\mid v_{2}=0} .
$$

In order to compute the entries $\widetilde{\mathrm{G}}_{11}$ and $\widetilde{\mathrm{G}}_{22}$ it appears more convenient to slightly reorganise their expression.

Indeed, by substituing the second expression for $\mathrm{R}_{12}$ given in (2.15), using its independence on $\mathrm{p}_{2}$ and then by simplifying factors issuing from the adjoint action of $\mathrm{L}_{2}$ operators from its decomposition (2.5) and finally, by moving the $D_{\alpha}\left(\mathrm{p}_{1}\right)$ operators to the almost exteme left and right sides what can be done by using the intertwining relation (2.10), one gets

$$
\begin{aligned}
& \mathrm{Z}_{1} \cdot \mathrm{R}_{12}\left(u_{1}, u_{2} \mid 0\right) \mathrm{L}_{1}\left(u_{1}, u_{2}\right) \cdot \mathrm{Z}_{2}^{-1} \\
& \quad=\mathrm{Z}_{1} \cdot D_{u_{1}-v_{2}}\left(\mathrm{p}_{1}\right) \cdot \mathrm{L}_{1}\left(v_{2}, u_{1}\right) \cdot \mathrm{M}_{u_{2}}\left(\mathrm{x}_{2}\right) \cdot D_{u_{2}-v_{2}}\left(\omega_{1} \omega_{2} \mathrm{x}_{12}-\mathrm{i} \frac{\omega_{2}}{2} \sigma_{3}\right) \cdot \mathrm{M}_{v_{2}}^{-1}\left(\mathrm{x}_{2}\right) \cdot \mathrm{Z}_{2}^{-1} \cdot D_{u_{2}-v_{1}}\left(\mathrm{p}_{1}\right) .
\end{aligned}
$$

In order to read out from that the expression for $\widetilde{G}_{11}$ one should compose the rhs with the vectore $v_{+}^{t}$ and $v_{+}$as defined in (2.20). For doing so, it is useful to remark that

$$
\boldsymbol{v}_{+}^{t} \mathrm{Z}_{1} \cdot D_{u_{1}-v_{2}}\left(\mathrm{p}_{1}\right) \cdot \mathrm{L}_{1}\left(v_{2}, u_{1}\right)=D_{u_{1}-v_{2}}\left(\mathrm{p}_{1}\right)\left(U_{1},-U_{1}^{-1}\right) \quad \text { and } \quad \mathrm{M}_{v_{2}}^{-1}\left(\mathrm{x}_{2}\right) \cdot \mathrm{Z}_{2}^{-1} \boldsymbol{v}_{+}=\frac{1}{V_{2}+V_{2}^{-1}}\left(\begin{array}{c}
1 \\
-1
\end{array}\right) \text {. }
$$


Also, one has

$$
\mathrm{N}_{0}\left(\mathrm{x}_{1}\right) \cdot \mathrm{M}_{u_{2}}\left(\mathrm{x}_{2}\right) \cdot D_{u_{2}-v_{2}}\left(\omega_{1} \omega_{2} \mathrm{x}_{12}-\mathrm{i} \frac{\omega_{2}}{2} \sigma_{3}\right)\left(\begin{array}{c}
1 \\
-1
\end{array}\right)=-\left(\begin{array}{c}
\mathrm{m} \\
\mathrm{m}
\end{array}\right)
$$

with

$$
\mathrm{m}=\left(U_{2}+U_{2}^{-1} \mathrm{e}^{2 \pi \omega_{2} \mathrm{x}_{21}}\right) \cdot D_{u_{2}}\left(\omega_{1} \omega_{2} \mathrm{x}_{12}-\mathrm{i} \frac{\omega_{2}}{2}\right)+\left(U_{2}^{-1}+U_{2} \mathrm{e}^{2 \pi \omega_{2} \mathrm{x}_{21}}\right) \cdot D_{u_{2}}\left(\omega_{1} \omega_{2} \mathrm{x}_{12}+\mathrm{i} \frac{\omega_{2}}{2}\right) .
$$

By using the transmutation properties of $D_{\alpha}$ functions (B.13), one recasts $m$ in the form

$$
\mathrm{m}=2 \mathrm{e}^{\pi \omega_{2} \mathrm{x}_{21}} \cdot D_{u_{2}+\mathrm{i} \frac{\omega_{2}}{2}}\left(\omega_{1} \omega_{2} \mathrm{x}_{12}\right) .
$$

All of this leads to

$$
\left.\widetilde{\mathrm{G}}_{11}\right|_{v_{2}=0}=\mathrm{i} D_{u_{1}}\left(\mathrm{p}_{1}\right)\left(U_{1},-U_{1}^{-1}\right) \mathrm{e}^{-\frac{\pi}{\omega_{1}}\left(\mathrm{p}_{1}-\mathrm{i} \frac{\Omega}{2}\right) \sigma_{3}}\left(\begin{array}{l}
1 \\
1
\end{array}\right) \mathrm{e}^{\pi \omega_{2} \mathrm{x}_{21}} \cdot D_{u_{2}+\mathrm{i} \frac{\omega_{2}}{2}}\left(\omega_{1} \omega_{2} \mathrm{x}_{12}\right) \cdot D_{u_{2}-v_{1}}\left(\mathrm{p}_{1}\right) .
$$

By taking the scalar products explicitly and then using, again, transmutation properties (B.13), one arrives to

$$
\left.\widetilde{\mathrm{G}}_{11}\right|_{v_{2}=0}=-\mathrm{e}^{-\pi \omega_{2} \mathrm{x}_{1}} \mathrm{R}_{12}\left(u_{1}+\mathrm{i} \frac{\omega_{2}}{2}, u_{2}+\mathrm{i} \frac{\omega_{2}}{2} \mid 0\right) \cdot \mathrm{e}^{\pi \omega_{2} \mathrm{x}_{2}} .
$$

Quite similarly, one gets

$$
\left.\widetilde{\mathrm{G}}_{11}\right|_{v_{2}=0}=4 \sinh \left[\frac{2 \pi}{\omega_{1}} u_{1}\right] \sinh \left[\frac{2 \pi}{\omega_{1}} u_{2}\right] \cdot \mathrm{e}^{\pi \omega_{2} \mathrm{x}_{1}} \mathrm{R}_{12}\left(u_{1}-\mathrm{i} \frac{\omega_{2}}{2}, u_{2}-\mathrm{i} \frac{\omega_{2}}{2} \mid 0\right) \cdot \mathrm{e}^{-\pi \omega_{2} \mathrm{x}_{2}} .
$$

The triangular structure in (3.36) allows one to explicitly take products and leads to the relation

$$
\mathrm{Z}_{1} \cdot\left(\mathrm{R}_{12} \cdots \mathrm{R}_{N 0}\right)\left(u_{1}, u_{2} \mid v_{2}\right) \mathrm{T}_{N}\left(u_{1}, u_{2}\right) \cdot \mathrm{Z}_{0}^{-1}=\mathrm{e}^{-\pi \omega_{2} \mathrm{x}_{1} \sigma_{3}}\left(\begin{array}{cc}
C_{\uparrow} & * \\
0 & C_{\downarrow}
\end{array}\right) \mathrm{e}^{\pi \omega_{2} \mathrm{x}_{0} \sigma_{3}}
$$

There, $\mathrm{T}_{N}\left(u_{1}, u_{2}\right)=\mathrm{L}_{1}\left(u_{1}, u_{2}\right) \cdots \mathrm{L}_{N}\left(u_{1}, u_{2}\right)$ is the monodromy matrix and we agree upon

$$
C_{\uparrow}=(-1)^{N}\left(\mathrm{R}_{12} \cdots \mathrm{R}_{N 0}\right)\left(u_{1}+\mathrm{i} \frac{\omega_{2}}{2}, u_{2}+\mathrm{i} \frac{\omega_{2}}{2} \mid 0\right)
$$

and

$$
C_{\downarrow}=\left\{4 \sinh \left[\frac{2 \pi}{\omega_{1}} u_{1}\right] \sinh \left[\frac{2 \pi}{\omega_{1}} u_{2}\right]\right\}^{N} \cdot\left(\mathrm{R}_{12} \cdots \mathrm{R}_{N 0}\right)\left(u_{1}-\mathrm{i} \frac{\omega_{2}}{2}, u_{2}-\mathrm{i} \frac{\omega_{2}}{2} \mid 0\right) .
$$

In order to deduce from (3.45) the action of the $\mathrm{B}_{N}$ operator, one needs to exchange the position of the monodromy matrix and the string of partial R-operators. The intertwining relation (2.16) leads to

$$
\begin{aligned}
\left(\mathrm{R}_{12} \cdots \mathrm{R}_{N 0}\right)\left(u_{1}, u_{2} \mid v_{2}\right) \mathrm{L}_{1}\left(u_{1}, u_{2}\right) \cdots & \mathrm{L}_{N}\left(u_{1}, u_{2}\right) \mathrm{L}_{0}\left(u_{1}, v_{2}\right) \\
& =\mathrm{L}_{1}\left(u_{1}, v_{2}\right) \mathrm{L}_{2}\left(u_{1}, u_{2}\right) \cdots \mathrm{L}_{0}\left(u_{1}, u_{2}\right)\left(\mathrm{R}_{12} \cdots \mathrm{R}_{N 0}\right)\left(u_{1}, u_{2} \mid v_{2}\right) .
\end{aligned}
$$

Then, upon using the relation (2.18) one obtains

$$
\begin{aligned}
& \mathrm{e}^{-2 \mathrm{i} \pi \epsilon\left(v_{2}-u_{2}\right) \mathrm{x}_{1}} \cdot\left(\mathrm{R}_{12} \cdots \mathrm{R}_{N 0}\right)\left(u_{1}, u_{2} \mid\right. \\
& \left.\quad v_{2}\right) \cdot v_{\epsilon}^{t} \mathrm{~T}_{N}\left(u_{1}, u_{2}\right) \mathrm{L}_{0}\left(u_{1}, v_{2}\right) \\
& \quad=v_{\epsilon}^{t} \mathrm{~T}_{N}\left(u_{1}, u_{2}\right) \mathrm{L}_{0}\left(u_{1}, u_{2}\right) \mathrm{e}^{-2 \mathrm{i} \pi \epsilon\left(v_{2}-u_{2}\right) \mathrm{x}_{1}}\left(\mathrm{R}_{12} \cdots \mathrm{R}_{N 0}\right)\left(u_{1}, u_{2} \mid v_{2}\right)
\end{aligned}
$$


Finally, upon using the independence on $\mathrm{p}_{0}$ of $\mathrm{R}_{N 0}$ and inserting the explicit expression for the $\mathrm{L}_{0}$ operators, one arrives to

$$
\begin{aligned}
& \mathrm{e}^{-2 \mathrm{i} \pi \epsilon\left(v_{2}-u_{2}\right) \mathrm{x}_{1}} \cdot\left(\mathrm{R}_{12} \cdots \mathrm{R}_{N 0}\right)\left(u_{1}, u_{2} \mid v_{2}\right) \cdot v_{\epsilon}^{t} \mathrm{~T}_{N}\left(u_{1}, u_{2}\right) \\
& =v_{\epsilon}^{t} \mathrm{~T}_{N}\left(u_{1}, u_{2}\right) \mathrm{e}^{-2 \mathrm{i} \pi \epsilon\left(v_{2}-u_{2}\right) \mathrm{x}_{1}}\left(\mathrm{R}_{12} \cdots \mathrm{R}_{N-1 N}\right)\left(u_{1}, u_{2} \mid v_{2}\right) \\
& \quad \times D_{u_{1}-v_{2}}\left(\mathrm{p}_{N}\right) \cdot \mathrm{M}_{u_{2}}\left(\mathrm{x}_{0}\right) \cdot D_{u_{2}-v_{2}}\left(\omega_{1} \omega_{2} \mathrm{x}_{N 0}-\mathrm{i} \frac{\omega_{2}}{2} \sigma_{3}\right) \cdot \mathrm{M}_{v_{2}}^{-1}\left(\mathrm{x}_{0}\right) \cdot D_{u_{2}-u_{1}}\left(\mathrm{p}_{N}\right) .
\end{aligned}
$$

This last identity implies that one may recast (3.45) in the form

$$
\begin{aligned}
v_{+}^{t} \mathrm{~T}_{N}\left(u_{1}, u_{2}\right) \mathrm{e}^{2 \mathrm{i} \pi u_{2} \mathrm{x}_{1}}\left(\mathrm{R}_{12} \cdots \mathrm{R}_{N-1 N}\right)\left(u_{1}, u_{2} \mid 0\right)=\mathrm{e}^{\mathrm{i} \pi\left(2 u_{2}+\mathrm{i} \omega_{2}\right) \mathrm{x}_{1}}\left(C_{\uparrow}, *\right) \mathrm{e}^{\pi \omega_{2} \mathrm{x}_{0} \sigma_{3}} \\
\quad \times D_{u_{1}-u_{2}}\left(\mathrm{p}_{N}\right) \cdot \mathrm{Z}_{0} \cdot \mathrm{M}_{0}\left(\mathrm{x}_{0}\right) \cdot D_{-u_{2}}\left(\omega_{1} \omega_{2} \mathrm{x}_{N 0}-\mathrm{i} \frac{\omega_{2}}{2} \sigma_{3}\right) \cdot \mathrm{M}_{u_{2}}^{-1}\left(\mathrm{x}_{0}\right) \cdot D_{-u_{1}}\left(\mathrm{p}_{N}\right) .
\end{aligned}
$$

Evaluating this expression on $\boldsymbol{v}_{-}$, acting with it on the function 1 in the space 0 and using that

$$
\lim _{x_{0} \rightarrow+\infty}\left\{\mathrm{e}^{-\pi \omega_{2} x_{0}} \cdot D_{u_{2}+\mathrm{i} \frac{\omega_{2}}{2}}\left(\omega_{1} \omega_{2} x_{N 0}\right) \cdot D_{-u_{2}}\left(\omega_{1} \omega_{2} x_{N 0}-\mathrm{i} \frac{\omega_{2}}{2} \sigma_{3}\right)\right\}=\mathrm{e}^{-\pi \omega_{2} x_{N}} U_{2}^{-\sigma_{3}},
$$

leads to

$$
\mathrm{B}_{N}\left(y-\mathrm{i} \omega_{1}\right) \cdot \gamma_{N}\left(u_{1}, u_{2}\right)=(-1)^{N+1} \gamma_{N}\left(u_{1}+\mathrm{i} \frac{\omega_{2}}{2}, u_{2}+\mathrm{i} \frac{\omega_{2}}{2}\right) \cdot D_{u_{1}+\mathrm{i} \frac{\omega_{2}}{2}}\left(\mathrm{p}_{N}\right) \mathrm{e}^{-\pi \omega_{2} \mathrm{x}_{N}} D_{-u_{1}}\left(\mathrm{p}_{N}\right)
$$

where we used that the condition $v_{2}=0$ is equivalent to $\lambda=y-\mathrm{i} \omega_{1}$ and we have set

$$
\gamma_{N}\left(u_{1}, u_{2}\right)=\mathrm{e}^{2 \mathrm{i} \pi u_{2} \mathrm{x}_{1}}\left(\mathrm{R}_{12} \cdots \mathrm{R}_{N-1 N}\right)\left(u_{1}, u_{2} \mid 0\right) .
$$

Finally, acting on the function $\mathrm{e}^{-2 \mathrm{i} \pi y_{+}^{\star} x_{N}}$ on the $N^{\text {th }}$ space produces the $\Lambda$-operator on the lhs and one gets, by recombining the factors

$$
\mathrm{B}_{N}\left(y-\mathrm{i} \omega_{1}\right) \cdot \Lambda_{y,-}^{(N)}=(-1)^{N+1}\left(\frac{\mathcal{A}\left(\left(y+\mathrm{i} \omega_{2}\right)_{+}\right)}{\mathcal{A}\left(y_{+}\right)}\right)^{N-1} D_{u_{1}+\mathrm{i} \frac{\omega_{2}}{2}}\left(y_{+}^{\star}-+\mathrm{i} \frac{\omega_{2}}{2}\right) D_{-u_{1}}\left(y_{+}^{\star}\right) \cdot \Lambda_{y+\mathrm{i} \omega_{2},-}^{(N)} .
$$

Upon observing that one has $u_{1}=y_{+}$, a straightforward calculation leads to

$$
\mathrm{B}_{N}\left(y-\mathrm{i} \omega_{1}\right) \cdot \Lambda_{y,-}^{(N)}=(-1)^{N-1} b(y) \Lambda_{y+\mathrm{i} \omega_{2},-}^{(N)}
$$

hence yielding the claim upon observing that $\mathrm{B}_{N}\left(y-\mathrm{i} \omega_{1}\right)=(-1)^{N-1} \mathrm{~B}_{N}(y)$.

For the statement of the next result, it appears convenient to keep track of the $\kappa$ dependence of the operator $\Lambda_{y,-}^{(N)}$ and denote it as $\Lambda_{y, \kappa,-}^{(N)}$.

\section{Lemma 3.2 One has the exchange relations}

$$
J_{N+1}\left(y_{-}-y_{+}\right) \cdot \Lambda_{y, \kappa,-}^{(N)}=D_{-\kappa}^{N}(y) \cdot \Lambda_{y,-\kappa,-}^{(N)} \cdot W_{N}\left(y_{-}-y_{+}\right) \cdot J_{N}\left(y_{-}-y_{+}\right)
$$

where we have introduced

$$
J_{N+1}(\alpha)=\prod_{a=1}^{N} D_{\alpha}\left(p_{a}\right) \quad \text { and } \quad W_{N+1}(\alpha)=J_{N+1}(\alpha) \mathrm{e}^{2 \mathrm{i} \pi \alpha x_{N}} \cdot \prod_{a=1}^{N} D_{\alpha}\left(\omega_{1} \omega_{2} \mathrm{x}_{a a+1}\right) \cdot \mathrm{e}^{2 \mathrm{i} \pi \alpha \mathrm{x}_{1}} .
$$

Also, it holds

$$
W_{N+1}\left(y_{-}-y_{+}\right) \cdot \Lambda_{y,-\kappa,-}^{(N)}=D_{-\kappa}(y) \cdot \Lambda_{y,-\kappa,-}^{(N)} \cdot W_{N}\left(y_{-}-y_{+}\right) .
$$

Note that, in the above definition, it is understood that

$$
\mathrm{W}_{1}(\alpha)=\mathrm{J}_{1}(\alpha)=D_{\alpha}\left(\mathrm{p}_{1}\right) .
$$

Proof - This is a direct consequence of a multiple application of the star-triangle relation and of the interchange relation of $D$ s and exponentials (B.22). 


\subsection{Elementary properties of a basic complete orthogonal system}

In this subsection we discuss the complete orthogonal system of generalised Eigenfunctions of the one-site operator $\mathrm{B}_{1}(\lambda)$ and establish its properties under specific action of $\Lambda_{y,-}^{(2)}$ operators.

Lemma 3.3 The functions

$$
\phi_{y}(x)=\int_{\mathbb{R}} \varpi(\kappa+t) \mathrm{e}^{2 i \pi t(x+y)} \cdot \mathrm{d} t
$$

are self-dual under the exchange $\omega_{1} \leftrightarrow \omega_{2}$ and satisfy to

$$
B_{1}(\lambda) \cdot \phi_{y}(x)=\mathrm{e}^{2 \pi \omega_{2} y} \phi_{y}(x)
$$

The family $\left\{\phi_{y}(x)\right\}$ forms a complete orthogonal system on $L^{2}(\mathbb{R})$, viz.

$$
\int_{\mathbb{R}} \phi_{y^{\prime}}^{*}(x) \cdot \phi_{y}(x) \cdot \mathrm{d} x=\delta\left(y^{\prime}-y\right) \quad \text { and } \quad \int_{\mathbb{R}} \phi_{y}^{*}\left(x^{\prime}\right) \cdot \phi_{y}(x) \cdot \mathrm{d} y=\delta\left(x^{\prime}-x\right) .
$$

Proof - The generalised spectral problem for the operator $\mathrm{B}_{1}(\lambda)$ associated with the generalised Eigenvalue $\mathrm{e}^{2 \pi \omega_{2} y}$ can be recast in the form of the below finite-difference equation

$$
2 \mathrm{i} \sinh \left[\frac{\pi}{\omega_{1}}\left(\kappa+\mathrm{p}+\mathrm{i} \frac{\tau}{2}\right)\right] \cdot \phi_{y}(x)=\mathrm{e}^{2 \pi \omega_{2}(x+y)} \phi_{y}(x) .
$$

One possible solution is given by $\phi_{y}(x)=\phi_{0}(x+y)$. Thus we focus on $\phi_{0}$. Passing to the Fourier space, one gets the below finite difference equation satisfied by the Fourier transform

$$
\mathcal{F}\left[\phi_{0}\right]\left(t+\mathrm{i} \omega_{2}\right)=2 \mathrm{i} \sinh \left[\frac{\pi}{\omega_{1}}\left(\kappa+t+\mathrm{i} \frac{\tau}{2}\right)\right] \mathcal{F}\left[\phi_{0}\right](t) .
$$

This equation, along with its dual, is easily solved in terms of the dilogarithm leading, all-in-all, to the claim. c.f. (B.2). The completeness and orthogonality follows from straightforward handlings. In particular, these entail that each generalised Eigenvalue of $B_{1}(\lambda)$ appears with multiplicity one.

Lemma 3.4 For $y, t, t_{0} \in \mathbb{R}$, it holds

$$
\left(\Lambda_{y,+}^{(2)}\right)^{\dagger} \cdot \Lambda_{t,+}^{(2)} \cdot \phi_{t_{0}}=\delta(y-t) \frac{\mathrm{e}^{-2 \pi \Omega t_{0}}}{\left(\omega_{1} \omega_{2}\right)^{\frac{3}{2}}} \phi_{t_{0}} .
$$

Proof-

One has the representation

$$
\begin{aligned}
\left(\Lambda_{y,+}^{(2)}\right)^{\dagger} \cdot \Lambda_{t,+}^{(2)} & =\frac{1}{\omega_{1} \omega_{2}} \mathcal{A}\left(\begin{array}{c}
\left(-y_{+}^{*}\right)^{\star} \\
t_{+}
\end{array}\right) \\
& \times \operatorname{tr}_{2}\left[\mathrm{e}^{-2 \mathrm{i} \pi\left(y_{+}^{\star}\right)^{*} \mathrm{x}_{2}} D_{-y_{+}^{*}}\left(\omega_{1} \omega_{2} \mathrm{x}_{12}\right) D_{-y_{-}^{*}}\left(\mathrm{p}_{1}\right) \cdot \mathrm{e}^{2 \mathrm{i} \pi\left(t_{-}-y_{-}^{*}\right) \mathrm{x}_{1}} \cdot D_{t_{-}}\left(\mathrm{p}_{1}\right) \cdot D_{t_{+}}\left(\omega_{1} \omega_{2} \mathrm{x}_{12}\right) \mathrm{e}^{2 \mathrm{i} \pi t_{+}^{\star} \mathrm{x}_{2}}\right]
\end{aligned}
$$


where the trace runs through the second quantum space. Here, we remind that we use the hypergeometric-like notation (A.3). In virtue of (B.25) the expression can be recast as

$$
\begin{aligned}
\left(\Lambda_{y,+}^{(2)}\right)^{\dagger} \cdot \Lambda_{t,+}^{(2)} \cdot \mathcal{A}\left(\begin{array}{c}
t_{+} \\
\left(-y_{+}^{*}\right)^{\star}
\end{array}\right) \cdot \omega_{1} \omega_{2} \\
=\operatorname{tr}_{2}\left[\mathrm{e}^{2 \mathrm{i} \pi\left[t_{-} \mathrm{x}_{1}-\left(y_{+}^{\star}\right)^{*} \mathrm{x}_{2}\right]} D_{-y_{+}^{*}}\left(\omega_{1} \omega_{2} \mathrm{x}_{12}\right) D_{t_{-}-y_{-}^{*}}\left(\mathrm{p}_{1}\right) \cdot D_{t_{+}}\left(\omega_{1} \omega_{2} \mathrm{x}_{12}\right) \mathrm{e}^{2 \mathrm{i} \pi\left[t_{+}^{\star} \mathrm{x}_{2}-y_{-}^{*} \mathrm{x}_{1}\right]}\right]
\end{aligned}
$$

Then, invoking the star-triangle relation (B.24) one obtains

$$
\begin{aligned}
& \left(\Lambda_{y,+}^{(2)}\right)^{\dagger} \cdot \Lambda_{t,+}^{(2)} \cdot \mathcal{A}\left(\begin{array}{c}
t_{+} \\
\left(-y_{+}^{*}\right)^{\star}
\end{array}\right) \cdot \omega_{1} \omega_{2} \\
& \quad=\operatorname{tr}_{2}\left[\mathrm{e}^{2 \mathrm{i} \pi\left[t_{-} \mathrm{x}_{1}-\left(y_{+}^{\star}\right)^{*} \mathrm{x}_{2}\right]} D_{t_{+}}\left(\mathrm{p}_{1}\right) D_{t_{-}-y_{-}^{*}}\left(\omega_{1} \omega_{2} \mathrm{x}_{12}\right) \cdot D_{-y_{+}^{*}}\left(\mathrm{p}_{1}\right) \mathrm{e}^{2 \mathrm{i} \pi\left(t_{+}^{\star} \mathrm{x}_{2}-y_{-}^{*} \mathrm{x}_{1}\right)}\right] \\
& =\mathrm{e}^{2 \mathrm{i} \pi t_{-} \mathrm{x}_{1}} \cdot D_{t_{+}}\left(\mathrm{p}_{1}\right) \cdot \mathrm{e}^{-2 \mathrm{i} \pi\left(y_{+}^{\star}\right)^{*} \mathrm{x}_{1}} \cdot \operatorname{tr}_{2}\left[\mathrm{e}^{-2 \mathrm{i} \pi\left[t_{+}^{\star}-\left(y_{+}^{\star}\right)^{*}\right] \mathrm{x}_{12}} D_{t_{-}-y_{-}^{*}}\left(\omega_{1} \omega_{2} \mathrm{x}_{12}\right)\right] \cdot \mathrm{e}^{2 \mathrm{i} \pi t_{+}^{\star} \mathrm{x}_{1}} \cdot D_{-y_{+}^{*}}\left(\mathrm{p}_{1}\right) \cdot \mathrm{e}^{-2 \mathrm{i} \pi y_{-}^{*} \mathrm{x}_{1}} \cdot
\end{aligned}
$$

At this stage one can already take the trace over the second space. However, for this purpose, one needs to regularise the integral appropriately. Indeed, one ends up with the below integral

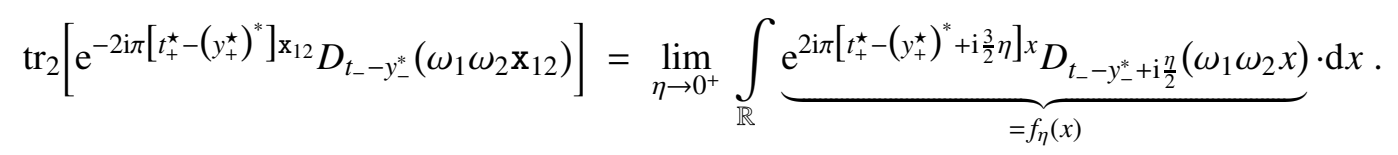

The above regularisation is such that $f_{\eta} \in L^{1}(\mathbb{R})$. Indeed, one has

$$
\left\{\begin{array}{c}
t_{+}^{\star}-\left(y_{+}^{\star}\right)^{*}=\frac{1}{2}(y-t)-\mathrm{i} \frac{\Omega}{2} \\
t_{-}-y_{-}^{*}=\frac{1}{2}(t-y)-\mathrm{i} \frac{\Omega}{2}
\end{array} \quad \text { viz. } \quad\left[t_{-}-y_{-}^{*}+\mathrm{i} \frac{\eta}{2}\right]^{\star}=\frac{y-t}{2}-\mathrm{i} \frac{\eta}{2}\right.
$$

and hence

$$
f_{\eta}(x) \underset{x \rightarrow+\infty}{\sim} \mathrm{e}^{2 \mathrm{i} \pi[y-t+\mathrm{i} \eta] x} \quad f_{\eta}(x) \underset{x \rightarrow-\infty}{\sim} \mathrm{e}^{2 \mathrm{i} \pi[-\mathrm{i} \Omega+2 \mathrm{i} \eta] x} .
$$

The integral in the rhs of (3.70) can be taken in closed form by means of (B.16). This leads, after some reorganisations, to

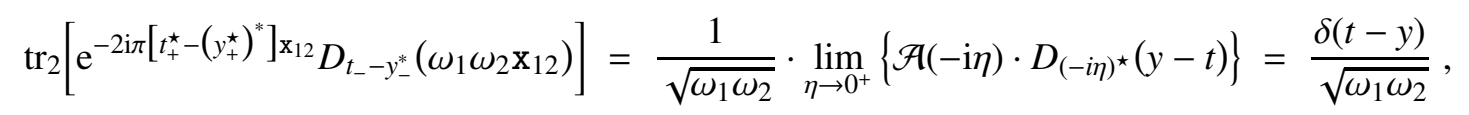

where we used (B.19). Then, inserting this result into (3.69) and observing that

$$
t_{+}^{\star}-\left(y_{+}^{\star}\right)^{*}=\frac{y-t}{2}-\mathrm{i} \frac{\Omega}{2} \quad, \quad t_{+}-y_{+}^{*}=\frac{t-y}{2}-\mathrm{i} \frac{\Omega}{2}
$$

one concludes that two quantities coincide when $t=y$. Further $\left(-y_{+}^{*}\right)^{\star}=y_{+}$what ensures that the $\mathfrak{A}$ factors in 
(3.69) cancel out. All-in-all, one gets that

$$
\begin{aligned}
\left(\Lambda_{y,+}^{(2)}\right)^{\dagger} \cdot \Lambda_{t,+}^{(2)} & =\frac{\delta(t-y)}{\left(\omega_{1} \omega_{2}\right)^{\frac{3}{2}}} \cdot \mathrm{e}^{2 \mathrm{i} \pi t_{-} \mathrm{x}_{1}} \cdot D_{t_{+}}\left(\mathrm{p}_{1}\right) \cdot \mathrm{e}^{2 \mathrm{i} \pi\left(t_{+}-y_{+}^{*}\right) \mathrm{x}_{1}} \cdot D_{-y_{+}^{*}}\left(\mathrm{p}_{1}\right) \cdot \mathrm{e}^{-2 \mathrm{i} \pi y_{-}^{*} \mathrm{x}_{1}} \\
& =\frac{\delta(t-y)}{\left(\omega_{1} \omega_{2}\right)^{\frac{3}{2}}} \cdot \mathrm{e}^{2 \mathrm{i} \pi\left(t_{-}-y_{+}^{*}\right) \mathrm{x}_{1}} \cdot D_{t_{+}-y_{+}^{*}}\left(\mathrm{p}_{1}\right) \cdot \mathrm{e}^{2 \mathrm{i} \pi\left(t_{+}-y_{-}^{*}\right) \mathrm{x}_{1}} \\
& =\frac{\delta(t-y)}{\left(\omega_{1} \omega_{2}\right)^{\frac{3}{2}}} \cdot \mathrm{e}^{2 \mathrm{i} \pi\left(t_{-}+t_{+}-y_{-}^{*}-y_{+}^{*}\right) \mathrm{x}_{1}} \cdot D_{t_{+}-y_{+}^{*}}\left(\mathrm{p}_{1}+t_{+}-y_{-}^{*}\right) \\
& =\frac{\delta(t-y)}{\left(\omega_{1} \omega_{2}\right)^{\frac{3}{2}}} \cdot \mathrm{e}^{-2 \mathrm{i} \pi \mathrm{i} \Omega \mathrm{x}_{1}} \cdot D_{-\mathrm{i} \frac{\Omega}{2}}\left(\mathrm{p}_{1}+\kappa-\mathrm{i} \frac{\Omega}{2}\right) \\
& =\frac{\delta(t-y)}{\left(\omega_{1} \omega_{2}\right)^{\frac{3}{2}}} \cdot \mathrm{e}^{-2 \mathrm{i} \pi \mathrm{i} \Omega \mathrm{x}_{1}} \varpi\left(\begin{array}{c}
\kappa-\mathrm{i} \Omega+\mathrm{p}_{1} \\
\kappa+\mathrm{p}_{1}
\end{array}\right) .
\end{aligned}
$$

It then solely remains to represent $\phi_{t_{0}}$ as

$$
\phi_{t_{0}}(x)=\int_{\mathbb{R}} \varpi(\kappa+y+\mathrm{i} \Omega) \mathrm{e}^{2 \mathrm{i} \pi(y+\mathrm{i} \Omega)\left(x+t_{0}\right)} \cdot \mathrm{d} y .
$$

Then by moving the action of $\left(\Lambda_{y,+}^{(2)}\right)^{\dagger} \cdot \Lambda_{t,+}^{(2)}$ under the integral sign one gets the claim.

\subsection{The orthogonal sets of Eigenfunctions of $\mathbf{A}_{N}(\lambda), \mathbf{B}_{N}(\lambda)$}

We are now in position to present a set of generalised Eigenfunctions associated with the operators $\mathrm{A}_{N}(\lambda)$ and $\mathrm{B}_{N}(\lambda)$. Then, we establish that these Eigenfunctions form, in each case, an orthogonal system.

Proposition 3.4 The functions

$$
\varphi_{\boldsymbol{y}_{N}}^{(-)}\left(\boldsymbol{x}_{N}\right)=\left(\Lambda_{y_{N},-}^{(N)} \cdots \Lambda_{y_{1},-}^{(1)}\right)\left(\boldsymbol{x}_{N}\right)
$$

and

$$
\varphi_{y_{0}, \boldsymbol{y}_{N-1}}^{(+)}\left(\boldsymbol{x}_{N}\right)=\mathrm{e}^{(N-1) \pi \Omega y_{0}} \cdot\left(\omega_{1} \omega_{2}\right)^{\frac{3}{4}(N-1)} \cdot\left(\Lambda_{y_{N-1},+}^{(N)} \cdots \Lambda_{y_{1},+}^{(2)} \cdot \phi_{y_{0}}\right)\left(\boldsymbol{x}_{N}\right)
$$

are, respectively, symmetric functions of $\boldsymbol{y}_{N}$ and $\boldsymbol{y}_{N-1}$ and satisfy to

$$
A_{N}(\lambda) \cdot \varphi_{\boldsymbol{y}_{N}}^{(-)}\left(\boldsymbol{x}_{N}\right)=\prod_{a=1}^{N}\left\{-2 \mathrm{i} \sinh \left[\frac{\pi}{\omega_{1}}\left(\lambda-y_{a}\right)\right]\right\} \cdot \varphi_{\boldsymbol{y}_{N}}^{(-)}\left(\boldsymbol{x}_{N}\right)
$$

and

$$
B_{N}(\lambda) \cdot \varphi_{y_{0}, \boldsymbol{y}_{N-1}}^{(+)}\left(\boldsymbol{x}_{N}\right)=\mathrm{e}^{2 \pi \omega_{2} y_{0}} \prod_{a=1}^{N-1}\left\{-2 \mathrm{i} \sinh \left[\frac{\pi}{\omega_{1}}\left(\lambda-y_{a}\right)\right]\right\} \cdot \varphi_{y_{0}, \boldsymbol{y}_{N-1}}^{(+)}\left(\boldsymbol{x}_{N}\right)
$$

Proof- 
The symmetry property in the $y$-variables follows from commutativity of the $\Lambda$ operators established in Proposition 3.2. The form of the action of $\mathrm{B}_{N}$ and $\mathrm{A}_{N}$ operators is a consequence of Lemmatae 3.3 and 3.1 and the fact that

$$
\left(\mathrm{A}_{1}(\lambda) \cdot \Lambda_{y,-}^{(1)}\right)\left(x_{1}\right)=-2 \mathrm{i} \sinh \left[\frac{\pi}{\omega_{1}}(\lambda-y)\right] \Lambda_{y,-}^{(1)}\left(x_{1}\right) \quad \text { where } \quad \Lambda_{y,-}^{(1)}(x)=\mathrm{e}^{2 \mathrm{i} \pi y x} .
$$

\section{Proposition 3.5 The families}

$$
\left\{\varphi_{\boldsymbol{y}_{N}}^{(-)}\left(\boldsymbol{x}_{N}\right)\right\}_{\boldsymbol{y}_{N} \in \mathbb{R}^{N}} \text { and }\left\{\varphi_{y_{0}, \boldsymbol{y}_{N-1}}^{(+)}\left(\boldsymbol{x}_{N}\right)\right\}_{\left(y_{0}, \boldsymbol{y}_{N-1}\right) \in \mathbb{R}^{N}}
$$

form an orthogonal system in $L^{2}\left(\mathbb{R}^{N}, \mathrm{~d}^{N} x\right)$, viz.

$$
\int_{\mathbb{R}^{N}}\left(\varphi_{\boldsymbol{y}_{N}^{\prime}}^{(-)}\left(\boldsymbol{x}_{N}\right)\right)^{*} \cdot \varphi_{\boldsymbol{y}_{N}}^{(-)}\left(\boldsymbol{x}_{N}\right) \cdot \mathrm{d}^{N} x=\frac{1}{\mu_{N}\left(\boldsymbol{y}_{N}\right)} \cdot \delta_{\mathrm{sym}}\left(\boldsymbol{y}_{N}-\boldsymbol{y}_{N}^{\prime}\right)
$$

and

$$
\int_{\mathbb{R}^{N}}\left(\varphi_{y_{0}^{\prime}, \boldsymbol{y}_{N-1}^{\prime}}^{(+}\left(\boldsymbol{x}_{N}\right)\right)^{*} \cdot \varphi_{y_{0}, \boldsymbol{y}_{N-1}}^{(+)}\left(\boldsymbol{x}_{N}\right) \cdot \mathrm{d}^{N} x=\frac{1}{\mu_{N-1}\left(\boldsymbol{y}_{N-1}\right)} \cdot \delta\left(y_{0}-y_{0}^{\prime}\right) \cdot \delta_{\mathrm{sym}}\left(\boldsymbol{y}_{N-1}-\boldsymbol{y}_{N-1}^{\prime}\right)
$$

where

$$
\mu_{N}\left(\boldsymbol{y}_{N}\right)=\prod_{a<b}^{N}\left\{4 \omega_{1} \omega_{2} \sinh \left[\frac{\pi}{\omega_{1}}\left(y_{a}-y_{b}\right)\right] \cdot \sinh \left[\frac{\pi}{\omega_{2}}\left(y_{a}-y_{b}\right)\right]\right\}
$$

and

$$
\delta_{\mathrm{sym}}\left(\boldsymbol{y}_{N}-\boldsymbol{y}_{N}^{\prime}\right)=\sum_{\sigma \in \mathbb{S}_{N}} \prod_{a=1}^{N} \delta\left(y_{a}-y_{\sigma(a)}^{\prime}\right) .
$$

Proof - We start by discussing the family $\varphi_{\boldsymbol{y}_{N}}^{(-)}$which is slightly easier to deal with. The orthogonality integral, which is to be understood in the sense of distributions, can be recast as

$$
\int_{\mathbb{R}^{N}}\left(\varphi_{\boldsymbol{t}_{N}}^{(-)}\left(\boldsymbol{x}_{N}\right)\right)^{*} \cdot \varphi_{\boldsymbol{y}_{N}}^{(-)}\left(\boldsymbol{x}_{N}\right) \cdot \mathrm{d}^{N} x=\left(\Lambda_{t_{1} ;-}^{(1)}\right)^{\dagger} \cdots\left(\Lambda_{t_{N} ;-}^{(N)}\right)^{\dagger} \cdot \Lambda_{y_{N} ;-}^{(N)} \cdots \Lambda_{y_{1} ;-}^{(1)} \cdot
$$

One now moves the Hermitian conjugate operators through the string of $\Lambda$ operators by means of the exchange relations (3.21). However, in order to exchange the operator $\left(\Lambda_{t ;-}^{(N)}\right)^{\dagger}$ with $\Lambda_{y ;-}^{(N)}$ one needs to ensure that $y \neq t$. This can be achieved by first, introducing the compactly supported smooth function $\varrho_{\epsilon}$ such that

$$
\varrho_{\epsilon}=1 \quad \text { on } \quad[-\epsilon ; \epsilon] \text { and } \varrho_{\epsilon}=0 \text { on } \mathbb{R} \backslash[-2 \epsilon ; 2 \epsilon] .
$$

Then, one considers

$$
g\left(\boldsymbol{y}_{N}, \boldsymbol{t}_{N}\right)=\sum_{\sigma \in \mathfrak{S}_{N}} \prod_{a \neq b}^{N}\left(1-\varrho_{\epsilon}\left(y_{a}-t_{\sigma(b)}\right)\right)
$$


which is uniformly away from 0 on $\mathcal{D}_{\epsilon} \times \mathcal{D}_{\epsilon}$ with $\mathcal{D}_{\epsilon}=\left\{\boldsymbol{y}_{N} \in \mathbb{R}^{N}: \min _{a \neq b}\left|y_{a}-y_{b}\right| \geq 7 \epsilon\right\}$. Thus, one gets a partition of the unity on $\mathcal{D}_{\epsilon} \times \mathcal{D}_{\epsilon}$ :

$$
\boldsymbol{S}_{\sigma}\left(\boldsymbol{y}_{N}, \boldsymbol{t}_{N}\right)=\frac{1}{g\left(\boldsymbol{y}_{N}, \boldsymbol{t}_{N}\right)} \prod_{a \neq b}^{N}\left(1-\varrho_{\epsilon}\left(y_{a}-t_{\sigma(b)}\right)\right) \quad \text { so that } \quad \sum_{\sigma \in \subseteq_{N}} \boldsymbol{S}_{\sigma}\left(\boldsymbol{y}_{N}, \boldsymbol{t}_{N}\right)=1 .
$$

Thus, one gets on $\mathcal{D}_{\epsilon} \times \mathcal{D}_{\epsilon}$ :

$$
\left(\Lambda_{t_{1} ;-}^{(1)}\right)^{\dagger} \cdots\left(\Lambda_{t_{N} ;-}^{(N)}\right)^{\dagger} \cdot \Lambda_{y_{N} ;-}^{(N)} \cdots \Lambda_{y_{1} ;-}^{(1)}=\sum_{\sigma \in \subseteq_{N}} \varsigma_{\mathrm{id}}\left(y_{N}, t_{N}^{(\sigma)}\right)\left(\Lambda_{t_{\sigma(N)} ;-}^{(1)}\right)^{\dagger} \cdots\left(\Lambda_{t_{\sigma(1)} ;-}^{(N)}\right)^{\dagger} \cdot \Lambda_{y_{N} ;-}^{(N)} \cdots \Lambda_{y_{1} ;-}^{(1)}
$$

where $\boldsymbol{t}_{N}^{(\sigma)}=\left(t_{\sigma(1)}, \cdots, t_{\sigma(N)}\right)$. One may already apply the exchange relation on the level of each summand (3.21) since the presence of $\varsigma_{\mathrm{id}}\left(\boldsymbol{y}_{N}, \boldsymbol{t}_{N}^{(\sigma)}\right)$ does ensure an appropriate distinctiveness of the variables, c.f. [19] for a rigorous treatment of this feature. This leads, in the $\epsilon \rightarrow 0^{+}$limit, to

$$
\begin{aligned}
\int_{\mathbb{R}^{N}}\left(\varphi_{\boldsymbol{t}_{N}}^{(-)}\left(\boldsymbol{x}_{N}\right)\right)^{*} \cdot & \varphi_{\boldsymbol{y}_{N}}^{(-)}\left(\boldsymbol{x}_{N}\right) \cdot \mathrm{d}^{N} x \\
& =\sum_{\sigma \in \mathfrak{S}_{N}} \prod_{a<b}^{N}\left\{\frac{1}{\omega_{1} \omega_{2}} \mathcal{A}\left(\left(t_{\sigma(a)}\right)_{+}^{\star}-\left(\left(y_{b}\right)_{+}^{\star}\right)^{*},\left(t_{\sigma(a)}\right)_{-}-\left(\left(y_{b}\right)_{-}\right)^{*}\right)\right\} \prod_{a=1}^{N}\left(\Lambda_{t_{\sigma(a)},-}^{(1)}\right)^{\dagger} \cdot \Lambda_{y_{a},-}^{(1)} .
\end{aligned}
$$

At this stage it solely remains to observe that for real $t, y$ one has

$$
\mathcal{A}\left(t_{+}^{\star}-\left(y_{+}^{\star}\right)^{*}, t_{-}-y_{-}^{*}\right)=\mathcal{A}\left(\frac{y-t}{2}-\mathrm{i} \frac{\Omega}{2}, \frac{t-y}{2}-\mathrm{i} \frac{\Omega}{2}\right)=\left\{4 \sinh \left[\frac{\pi}{\omega_{1}}(y-t)\right] \cdot \sinh \left[\frac{\pi}{\omega_{2}}(y-t)\right]\right\}^{-1}
$$

and also that

$$
\left(\Lambda_{t,-}^{(1)}\right)^{\dagger} \cdot \Lambda_{y,-}^{(1)}=\delta(y-t) .
$$

In the above handlings, we have omitted all technical details that need to be discussed so as to deal rigorously with the various steps of the calculations in the sense of distributions. The appropriate theory for that has been developed in [19] and we refer to this work for all the details leading to a rigorous treatment.

In the case of $\varphi_{y_{0}, y_{N-1}}^{(+)}$, the same kind of operations leads to

$$
\begin{aligned}
& \int_{\mathbb{R}^{N}}\left(\varphi_{t_{0}, t_{N-1}}^{(+)}\left(\boldsymbol{x}_{N}\right)\right)^{*} \cdot \varphi_{y_{0}, y_{N-1}}^{(+)}\left(\boldsymbol{x}_{N}\right) \cdot \mathrm{d}^{N} x=\mathrm{e}^{(N-1) \pi \Omega\left(t_{0}+y_{0}\right)} \cdot\left(\omega_{1} \omega_{2}\right)^{\frac{3}{2}(N-1)} \\
& \times \sum_{\sigma \in \mathfrak{S}_{N-1}} \prod_{a<b}^{N-1}\left\{\frac{1}{\omega_{1} \omega_{2}} \mathcal{A}\left(\left(t_{\sigma(a)}\right)_{+}^{\star}-\left(\left(y_{b}\right)_{+}^{\star}\right)^{*},\left(t_{\sigma(a)}\right)_{-}-\left(\left(y_{b}\right)_{-}\right)^{*}\right)\right\} \cdot\left(\phi_{t_{0}},\left(\Lambda_{t_{\sigma(N-1)},-}^{(2)}\right)^{\dagger} \Lambda_{y_{N-1},-}^{(2)} \cdots\left(\Lambda_{t_{\sigma(1)},-}^{(2)}\right)^{\dagger} \Lambda_{y_{1},-}^{(2)} \cdot \phi_{y_{0}}\right)_{L^{2}(\mathbb{R})} .
\end{aligned}
$$

Then, by trivial induction based on Lemma 3.4, one gets

$$
\begin{aligned}
\left(\phi_{t_{0}},\left(\Lambda_{t_{N-1},-}^{(2)}\right)^{\dagger} \Lambda_{y_{N-1},-}^{(2)} \cdots\left(\Lambda_{t_{1},-}^{(2)}\right)^{\dagger} \Lambda_{y_{1},-}^{(2)} \cdot \phi_{y_{0}}\right)_{L^{2}(\mathbb{R})} & \\
& =\frac{\mathrm{e}^{-(N-1) 2 \pi \Omega y_{0}}}{\left(\omega_{1} \omega_{2}\right)^{\frac{3}{2}(N-1)}} \cdot \prod_{a=1}^{N-1} \delta\left(t_{a}-y_{a}\right) \cdot\left(\phi_{t_{0}}, \phi_{y_{0}}\right)_{L^{2}(\mathbb{R})}=\frac{\mathrm{e}^{-(N-1) \pi \Omega\left(y_{0}+t_{0}\right)}}{\left(\omega_{1} \omega_{2}\right)^{\frac{3}{2}(N-1)}} \prod_{a=0}^{N-1} \delta\left(t_{a}-y_{a}\right)
\end{aligned}
$$

by virtue of the orthogonality of the system of functions $\phi_{y_{0}}, c f$. Lemma 3.3. 


\section{The Mellin-Barnes representation and completeness}

In this section, we construct the Mellin-Barnes representation for a family $\left\{\psi_{\boldsymbol{y}_{N}}^{(-)}\right\}_{\boldsymbol{y}_{N} \in \mathbb{R}^{N}}, \operatorname{resp} .\left\{\psi_{y_{0}, \boldsymbol{y}_{N-1}}^{(+)}\right\}_{y_{0} \in \mathbb{R}, \boldsymbol{y}_{N-1} \in \mathbb{R}^{N-1}}$, of generalised Eigenfunctions of the operator $\mathrm{A}_{N}(\lambda)$, resp. $\mathrm{B}_{N}(\lambda)$. We then use this integral representation to show that $\left\{\psi_{\boldsymbol{y}_{N}}^{(-)}\right\}_{\boldsymbol{y}_{N} \in \mathbb{R}^{N}}$, resp. $\left\{\psi_{y_{0}, \boldsymbol{y}_{N-1}}^{(+)}\right\}_{y_{0} \in \mathbb{R}, \boldsymbol{y}_{N-1} \in \mathbb{R}^{N-1}}$, forms a complete system on $L^{2}\left(\mathbb{R}^{N}, \mathrm{~d}^{N} x\right)$ in respect to an $L_{\text {sym }}^{2}\left(\mathbb{R}^{N}, \mathrm{~d} \mu_{N}\left(\boldsymbol{y}_{N}\right)\right)$, resp. $L_{\cdot \times \operatorname{sym}}^{2}\left(\mathbb{R} \times \mathbb{R}^{N-1}, \mathrm{~d} y_{0} \otimes \mathrm{d} \mu_{N-1}\left(\boldsymbol{y}_{N-1}\right)\right)$, integration of the $y$-variables. Here, the subscript sym refers to functions invariant under the natural action of the symmetric group $\mathfrak{\subseteq}_{N}$, resp. $\mathfrak{\subseteq}_{N-1}$.

\subsection{The Mellin-Barnes integral representation}

Given an $k$-dimensional vector $\boldsymbol{v}_{k}$, we agree upon the shorthand notation $\overline{\boldsymbol{v}}_{k}=\sum_{a=1}^{k} v_{a}$. Also, we shall denote by $\boldsymbol{e}_{k}$ the canonical unit base vector with 1 in the $k^{\text {th }}$ position and 0 everywhere else. Depending on the context, these vectors will belong to $\mathbb{R}^{N}$ or $\mathbb{R}^{N-1}$.

Proposition 4.1 Let $\boldsymbol{y}_{N} \in \mathbb{R}^{N}$. Define the functions $\psi_{\boldsymbol{y}_{N}}^{(-)}\left(\boldsymbol{x}_{N}\right)$ inductively by

$$
\psi_{\boldsymbol{y}_{N}}^{(-)}\left(\boldsymbol{x}_{N}\right)=\int_{C_{\boldsymbol{y}_{N}^{N-1}}^{N}} \psi_{\boldsymbol{w}_{N-1}}^{(-)}\left(\boldsymbol{x}_{N-1}\right) \psi_{\overline{\boldsymbol{y}}_{N}-\overline{\boldsymbol{w}}_{N-1}}^{(-)}\left(x_{N}\right) \Phi\left(\boldsymbol{w}_{N-1} \mid \boldsymbol{y}_{N}\right) \cdot \frac{\mathrm{d}^{N-1} w}{(N-1) !}
$$

and

$$
\psi_{y}^{(-)}(x)=\mathrm{e}^{2 \mathrm{i} \pi x y}
$$

Here

$$
\Phi\left(\boldsymbol{w}_{N-1} \mid \boldsymbol{y}_{N}\right)=\left(\frac{1}{\omega_{1} \omega_{2}}\right)^{N-1} \prod_{a=1}^{N} \frac{\varpi\left(y_{a}-\kappa\right)}{\varpi^{N-1}\left(y_{a}+\kappa\right)} \cdot \frac{\prod_{a=1}^{N-1} \varpi^{N-1}\left(w_{a}+\kappa\right)}{\varpi\left(\overline{\boldsymbol{y}}_{N}-\overline{\boldsymbol{w}}_{N-1}-\kappa\right)} \cdot \frac{\prod_{a=1}^{N-1} \prod_{b=1}^{N} \varpi\left(y_{b}-w_{a}-\mathrm{i} \frac{\Omega}{2}\right)}{\prod_{a \neq b}^{N-1} \varpi\left(w_{b}-w_{a}-\mathrm{i} \frac{\Omega}{2}\right)}
$$

and $C_{y_{N}}$ is a curve corresponding to small deformation of the real axis such that all the simple poles for each w-variable at

$$
w=y_{b}+\mathrm{i} \ell \omega_{1}+\mathrm{i} k \omega_{2}, \quad \text { with }(\ell, k) \in \mathbb{N}^{2} \text { and } b \in \llbracket 1 ; N \rrbracket
$$

are located above it.

Then, the functions $\psi_{y_{N}}^{(-)}$are well-defined, self-dual under $\omega_{1} \leftrightarrow \omega_{2}$ and satisfy

$$
A_{N}(\lambda) \cdot \psi_{\boldsymbol{y}_{N}}^{(-)}\left(\boldsymbol{x}_{N}\right)=a\left(\lambda \mid \boldsymbol{y}_{N}\right) \cdot \psi_{\boldsymbol{y}_{N}}^{(-)}\left(\boldsymbol{x}_{N}\right) \quad \text { with } \quad a\left(\lambda \mid \boldsymbol{y}_{N}\right)=\prod_{b=1}^{N}\left\{-2 \mathrm{i} \sinh \left[\frac{\pi}{\omega_{1}}\left(\lambda-y_{b}\right)\right]\right\}
$$

as well as

$$
\begin{aligned}
& B_{N}(\lambda) \cdot \psi_{y_{N}}^{(-)}\left(\boldsymbol{x}_{N}\right)=\sum_{k=1}^{N}\left\{b\left(y_{k}\right)\right\}^{N} \prod_{\substack{p=1 \\
\neq k}}^{N} \frac{\sinh \left[\frac{\pi}{\omega_{1}}\left(\lambda-y_{p}\right)\right]}{\sinh \left[\frac{\pi}{\omega_{1}}\left(y_{k}-y_{p}\right)\right]} \cdot \psi_{\boldsymbol{y}_{N}+\mathrm{i} \omega_{2} \boldsymbol{e}_{k}}^{(-)}\left(\boldsymbol{x}_{N}\right) \\
& C_{N}(\lambda) \cdot \psi_{\boldsymbol{y}_{N}}^{(-)}\left(\boldsymbol{x}_{N}\right)=\sum_{k=1}^{N}\left\{c\left(y_{k}\right)\right\}^{N} \prod_{\substack{p=1 \\
\neq k}}^{N} \frac{\sinh \left[\frac{\pi}{\omega_{1}}\left(\lambda-y_{p}\right)\right]}{\sinh \left[\frac{\pi}{\omega_{1}}\left(y_{k}-y_{p}\right)\right]} \cdot \psi_{\boldsymbol{y}_{N}-\mathrm{i} \omega_{2} \boldsymbol{e}_{k}}^{(-)}\left(\boldsymbol{x}_{N}\right)
\end{aligned}
$$


where

$$
b(\lambda)=-2 \mathrm{i} \sinh \left[\frac{\pi}{\omega_{1}}\left(\lambda+\kappa+\mathrm{i} \frac{\Omega}{2}\right)\right] \quad \text { and } \quad c(\lambda)=2 \mathrm{i} \sinh \left[\frac{\pi}{\omega_{1}}\left(\lambda-\kappa-\mathrm{i} \frac{\Omega}{2}\right)\right] .
$$

Further, given $\left(y_{0}, \boldsymbol{y}_{N-1}\right) \in \mathbb{R}^{N}$, the functions $\psi_{y_{0}, \boldsymbol{y}_{N-1}}^{(+)}\left(\boldsymbol{x}_{N}\right)$ defined as

$$
\psi_{y_{0}, \boldsymbol{y}_{N-1}}^{(+)}\left(\boldsymbol{x}_{N}\right)=\int_{C_{\boldsymbol{y}_{N}}^{N-1} \times C_{-y_{N}}} \psi_{\boldsymbol{w}_{N-1}}^{(-)}\left(\boldsymbol{x}_{N-1}\right) \psi_{w_{N}}^{(-)}\left(x_{N}\right) \Psi\left(\boldsymbol{w}_{N} \mid y_{0}, \boldsymbol{y}_{N-1}\right) \cdot \frac{\mathrm{d}^{N-1} w \otimes \mathrm{d} w_{N}}{(N-1) !}
$$

in which

$$
\begin{aligned}
& \Psi\left(\boldsymbol{w}_{N} \mid y_{0}, \boldsymbol{y}_{N-1}\right)=\left(\frac{1}{\omega_{1} \omega_{2}}\right)^{N-1} \cdot \frac{\mathrm{e}^{2 \mathrm{i} \pi y_{0} \overline{\boldsymbol{w}}_{N}} \varpi\left(w_{N}+\kappa\right)}{\prod_{a=1}^{N-1} \varpi^{N-1}\left(w_{a}+\kappa\right)} \cdot \prod_{a=1}^{N-1} \frac{\varpi\left(y_{a}-\kappa\right)}{\varpi^{N-1}\left(y_{a}+\kappa\right)} \\
& \times \prod_{a=1}^{N-1} \frac{\varpi\left(w_{a}+w_{N}+\mathrm{i} \frac{\Omega}{2}\right)}{\varpi\left(y_{a}+w_{N}+\mathrm{i} \frac{\Omega}{2}\right)} \cdot \frac{\prod_{a=1}^{N-1} \prod_{b=1}^{N-1} \varpi\left(y_{b}-w_{a}-\mathrm{i} \frac{\Omega}{2}\right)}{\prod_{a \neq b}^{N-1} \varpi\left(w_{b}-w_{a}-\mathrm{i} \frac{\Omega}{2}\right)},
\end{aligned}
$$

satisfy to

$$
B_{N}(\lambda) \cdot \psi_{y_{0}, y_{N-1}}^{(+)}\left(\boldsymbol{x}_{N}\right)=\mathrm{e}^{2 \pi \omega_{2} y_{0}} a\left(\lambda \mid \boldsymbol{y}_{N-1}\right) \cdot \psi_{y_{0}, \boldsymbol{y}_{N-1}}^{(+)}\left(\boldsymbol{x}_{N}\right)
$$

and

$$
\begin{array}{r}
A_{N}(\lambda) \cdot \psi_{y_{0}, y_{N-1}}^{(+)}\left(\boldsymbol{x}_{N}\right)=-\mathrm{i} a\left(\lambda \mid \boldsymbol{y}_{N-1}\right) \cdot\left\{\mathrm{e}^{\frac{\pi}{\omega_{1}}\left(\lambda+\overline{\boldsymbol{y}}_{N-1}\right)} \psi_{y_{0}+\frac{\mathrm{i}}{2 \omega_{1}}, \boldsymbol{y}_{N-1}}^{(+)}\left(\boldsymbol{x}_{N}\right)-\mathrm{e}^{-\frac{\pi}{\omega_{1}}\left(\lambda+\overline{\boldsymbol{y}}_{N-1}\right)} \psi_{y_{0}-\frac{\mathrm{i}}{2 \omega_{1}}, \boldsymbol{y}_{N-1}}^{(+)}\left(\boldsymbol{x}_{N}\right)\right\} \\
+\sum_{k=1}^{N-1}\left\{b\left(y_{k}\right)\right\}^{N} \prod_{\substack{p=1 \\
\neq k}}^{N-1} \frac{\sinh \left[\frac{\pi}{\omega_{1}}\left(\lambda-y_{p}\right)\right]}{\sinh \left[\frac{\pi}{\omega_{1}}\left(y_{k}-y_{p}\right)\right]} \cdot \psi_{y_{0}, \boldsymbol{y}_{N-1}+\mathrm{i} \omega_{2} \boldsymbol{e}_{k}}^{(+)}\left(\boldsymbol{x}_{N}\right)
\end{array}
$$

Proof - The proof goes by induction on $N$. One builds $\psi_{\boldsymbol{y}_{N}}^{(-)}\left(\boldsymbol{x}_{N}\right)$ as in (4.1) and assumes that the properties (4.5), (4.6) and (4.7) hold up to $N-1$. Then decomposing the monodromy matrix $\mathrm{T}_{N}(\lambda)=\mathrm{T}_{N-1}(\lambda) L_{N}^{(\kappa)}(\lambda)$ allows one to compute the action of the operator $\mathrm{A}_{N}, \mathrm{~B}_{N}$ and $\mathrm{C}_{N}$ on $\psi_{\boldsymbol{y}_{N}}^{(-)}\left(\boldsymbol{x}_{N}\right)$. The system of first order difference equations satisfied by $\Phi\left(\boldsymbol{w}_{N-1} \mid \boldsymbol{y}_{N}\right)$ then allows one to conclude that, indeed, the claimed form of the actions hold. See [26] or, in a closer setting to the present one, [15, 17]. Self-duality of $\psi_{\boldsymbol{y}_{N}}^{(-)}\left(\boldsymbol{x}_{N}\right)$ is manifest. The convergence of the integral can be established along the lines discussed in [11] for the analogous representation arising in the case of the quantum Toda chain.

\subsection{An auxiliary integral}

Prior to proving completeness, as already discussed in the introduction, we need to establish an auxiliary integral representation for the symmetric $\delta$-function in many variables. This identity appears as a central tool allowing to prove, in a simple and direct manner, the completeness of a system of functions defined through a MellinBarnes like representation. The technique for establishing this identity that we develop below thus emerges as 
an important tool for studying quantum integrable models with non-compact local spaces and solvable by the quantum separation of variables method.

Given $\boldsymbol{v}_{N-1}, \boldsymbol{w}_{N-1} \in \mathbb{R}^{N-1}$ and $\boldsymbol{\varepsilon}_{N} \in \mathbb{R}^{N}$ such that $\varepsilon_{a}>0$ for all $a$ and is small enough. Define $\mathscr{C}$ to be a curve obtained from $\mathbb{R}$ by a small deformation of $\mathbb{R}$ such that, for any $a=1, \ldots, N-1$ and $b=1, \ldots, N$ :

- the lattice $v_{a}-\mathrm{i} \varepsilon_{b}+\mathrm{i} \omega_{1} \mathbb{N}+\mathrm{i} \omega_{2} \mathbb{N}$ lies entirely above $\mathscr{C}$;

- the lattice $w_{a}+\mathrm{i} \varepsilon_{b}-\mathrm{i} \omega_{1} \mathbb{N}-\mathrm{i} \omega_{2} \mathbb{N}$ lies entirely below of $\mathscr{C}$.

Such a curve always exists provided that $\left\|\boldsymbol{\varepsilon}_{N}\right\|$ is small enough and the $\varepsilon_{a}$ 's are generic.

A very useful object occurring in the proof of completeness is given by the below family of integrals parameterised by $\boldsymbol{\varepsilon}_{N}$ as above:

$$
\mathscr{J}_{\boldsymbol{\varepsilon}_{N}}^{(N ; m)}\left(x \mid \boldsymbol{w}_{N-1}, \boldsymbol{v}_{N-1}\right)=\int_{\mathscr{C}^{m}} \mathrm{~d}^{m} y \int_{\mathbb{R}^{N-m}} \prod_{a=m+1}^{N} \mathrm{~d} y_{a} \cdot \mathcal{I}_{N}^{(m)}\left(\boldsymbol{\varepsilon}_{N} \mid x, \boldsymbol{y}_{N} ; \boldsymbol{w}_{N-1}, \boldsymbol{v}_{N-1}\right),
$$

with

$$
\mathcal{I}_{N}^{(m)}\left(\boldsymbol{\varepsilon}_{N} \mid x, \boldsymbol{y}_{N} ; \boldsymbol{w}_{N-1}, \boldsymbol{v}_{N-1}\right)=\frac{\mathrm{e}^{2 \mathrm{i} \pi \overline{\boldsymbol{y}}_{N} x}}{N !\left(\omega_{1} \omega_{2}\right)^{N-1}} \frac{\prod_{a=1}^{N-1} \prod_{b=1}^{N} \varpi\left(y_{b}-w_{a}-\mathrm{i} \frac{\Omega}{2}+\mathrm{i} \zeta_{b}^{(m)}, v_{a}-y_{b}-\mathrm{i} \frac{\Omega}{2}+\mathrm{i} \zeta_{b}^{(m)}\right)}{\prod_{a<b}^{N} \varpi\left(y_{b a}-\mathrm{i} \frac{\Omega}{2}+\mathrm{i} \zeta_{b}^{(m)}+\mathrm{i} \zeta_{a}^{(m)}, y_{a b}-\mathrm{i} \frac{\Omega}{2}-\mathrm{i} \zeta_{b}^{(m)}+\mathrm{i} \zeta_{a}^{(m)}\right)} .
$$

The sequence $\zeta_{a}^{(m)}$ is defined as

$$
\zeta_{a}^{(m)}=-\varepsilon_{a}, a=1, \ldots, m \text { and } \zeta_{a}^{(m)}=\left(\widetilde{\varepsilon_{N}}\right)_{a}^{(m)}=\varepsilon_{N+m+1-a}, a=m+1, \ldots, N .
$$

Also, we remind that $y_{a b}=y_{a}-y_{b}$ and we used hypergeometric-like notation for products of $\varpi$ factors , c.f. (A.3).

Proposition 4.2 For any $N \geq 1$ and $m \in \llbracket 0 ; N \rrbracket$, it holds, in the sense of distributions,

$$
\begin{aligned}
\lim _{\boldsymbol{\varepsilon}_{N} \rightarrow 0^{+}}\left\{\mathscr{J}_{\boldsymbol{\varepsilon}_{N}}^{(N ; m)}\left(x \mid \boldsymbol{w}_{N-1}, \boldsymbol{v}_{N-1}\right)\right\}=\prod_{a \neq b}^{N} \varpi\left(w_{a}-w_{b}-\mathrm{i} \frac{\Omega}{2}\right) \cdot \delta(x) \\
\times \delta_{\mathrm{sym}}\left(\boldsymbol{w}_{N-1}-\boldsymbol{v}_{N-1}\right) \cdot\left\{\begin{array}{cc}
1 & m=0 \\
\frac{1}{N} & m=1 \\
0 & m \in \llbracket 2 ; N \rrbracket
\end{array}\right.
\end{aligned}
$$

Proof-

The result is established by a triangular induction.

\section{- Initialisation}

It is obvious that

$$
\lim _{\varepsilon_{1} \rightarrow 0^{+}}\left\{\mathscr{J}_{\varepsilon_{1}}^{(1 ; a)}(x \mid \emptyset, \emptyset)\right\}=\delta(x), \quad \text { for } a \in\{0,1\} .
$$

Next, assume that the claim holds up to $N-1$ and for any $m \in \llbracket 0 ; N-1 \rrbracket$. 


\section{- Initialisation step at $N$ : the integral $\mathscr{J}_{\mathcal{E}_{N}}^{(N ; N)}$}

First of all, observe that the appropriate choice of signs of the regulators $\zeta_{a}^{(N)}$ in (4.14)-(4.15) makes the integrand

$$
\mathcal{I}_{N}^{(N)}\left(\boldsymbol{\varepsilon}_{N} \mid x, \boldsymbol{y}_{N} ; \boldsymbol{w}_{N-1}, \boldsymbol{v}_{N-1}\right)
$$

algebraically decreasing at $\infty$ along the lines $(\mathbb{R} \pm \mathrm{i} r)^{N}$, with $r>0$. This property is enough so as to allow for computing the integral by taking residues in the upper or lower complex plane -relatively to any variables- and conclude that, in the sense of distributions, the contours at $\infty$ do not contribute to the integral. Thus, in the sense of distributions, the integral can be taken by computing the residues of all the poles lying above $\mathscr{C}$-if $x \geq 0$ - or below $\mathscr{C}$-if $x \leq 0$-. Here, we only discuss the case when $x \geq 0$. In respect to each variable $y_{a}$, the integrand has simple poles at

$$
y_{a}=v_{b}-\mathrm{i} \epsilon_{a}+\mathrm{i} \ell_{a} \omega_{1}+\mathrm{i} k_{a} \omega_{2} \quad \text { with } \quad \ell_{a}, k_{a} \in \mathbb{N} \quad \text { and } \quad b \in \llbracket 1 ; N \rrbracket .
$$

Thus, one gets that, in the sense of distributions,

$$
\begin{aligned}
& \mathscr{J}_{\mathcal{\varepsilon}_{N}}^{(N ; N)}\left(x \mid \boldsymbol{w}_{N-1}, \boldsymbol{v}_{N-1}\right) \\
& =\sum_{\alpha \in \mathcal{M}_{N}} \sum_{\substack{\boldsymbol{\ell}_{N}, \boldsymbol{k}_{N} \\
\in \mathbb{N}^{N}}} \operatorname{Res}\left(I_{N}^{(N)}\left(\boldsymbol{\varepsilon}_{N} \mid x, \boldsymbol{y}_{N} ; \boldsymbol{w}_{N-1}, \boldsymbol{v}_{N-1}\right) \cdot \mathrm{d}^{N} y, \boldsymbol{y}_{N}=\boldsymbol{v}^{(\alpha)}+\mathrm{i} \boldsymbol{\ell}_{N} \omega_{1}+\mathrm{i} \boldsymbol{k}_{N} \omega_{2}-\mathrm{i} \boldsymbol{\varepsilon}_{N}\right) .
\end{aligned}
$$

The sums in (4.20) run through all maps $\alpha \in \mathcal{M}_{N}$, where $\mathcal{M}_{N}=\{\alpha: \llbracket 1 ; N \rrbracket \rightarrow \llbracket 1 ; N-1 \rrbracket\}$. Furthermore, given a map $\alpha: \llbracket 1 ; N \rrbracket \rightarrow \llbracket 1 ; N-1 \rrbracket$, we denote

$$
\boldsymbol{v}^{(\alpha)} \in \mathbb{R}^{N} \quad \text { the vector given by }\left(\boldsymbol{v}^{(\alpha)}\right)_{a}=v_{\alpha(a)} \quad \text { for } \quad a=1, \ldots, N .
$$

Once that the residues are computed, one may send $\varepsilon_{a} \rightarrow 0^{+}$for any $a=1, \ldots, N$. The expression for the residues of the dilogarithm (B.7) leads to

$$
\operatorname{Res}\left(\mathcal{I}_{N}^{(N)}\left(\mathbf{0} \mid x, \boldsymbol{y}_{N} ; \boldsymbol{w}_{N-1}, \boldsymbol{v}_{N-1}\right) \cdot \mathrm{d}^{N} y, \boldsymbol{y}_{N}=\boldsymbol{v}^{(\alpha)}+\mathrm{i} \boldsymbol{\ell}_{N} \omega_{1}+\mathrm{i} \boldsymbol{k}_{N} \omega_{2}\right)=P_{\boldsymbol{\ell}_{n} ; \boldsymbol{k}_{N}}^{(\alpha)} \cdot G_{\boldsymbol{\ell}_{n} ; \boldsymbol{k}_{N}}^{(\alpha)} \cdot \gamma_{\boldsymbol{\ell}_{n} ; \boldsymbol{k}_{N}}^{(\alpha)},
$$

in which

$$
\begin{aligned}
& \gamma_{\boldsymbol{\ell}_{n} ; \boldsymbol{k}_{N}}^{(\alpha)}=\frac{2^{N(N-1)}}{N ! \cdot\left(\omega_{1} \omega_{2}\right)^{\frac{N}{2}-1}} \prod_{a=1}^{N}\left\{(-1)^{k_{a} \ell_{a}}(2 \mathrm{i})^{\ell_{a}+k_{a}} \cdot \prod_{p=1}^{k_{a}} \sinh \left[\mathrm{i} p \pi \frac{\omega_{2}}{\omega_{1}}\right] \cdot \prod_{p=1}^{\ell_{a}} \sinh \left[\mathrm{i} p \pi \frac{\omega_{1}}{\omega_{2}}\right]\right\}^{-1}, \\
& P_{\boldsymbol{\ell}_{n} ; \boldsymbol{k}_{N}}^{(\alpha)}=\mathrm{e}^{2 \mathrm{i} \pi x\left(\overline{\boldsymbol{v}(\alpha)}+\mathrm{i} \overline{\boldsymbol{\ell}}_{N} \omega_{1}+\mathrm{i} \overline{\boldsymbol{k}}_{N} \omega_{2}\right)} \cdot \prod_{a=1}^{N-1} \prod_{b=1}^{N} \varpi\left(v_{\alpha(b)}-w_{a}-\mathrm{i} \frac{\Omega}{2}+\mathrm{i} \ell_{b} \omega_{1}+\mathrm{i} k_{b} \omega_{2}\right) .
\end{aligned}
$$

Note that above, we made use of the notation (A.2). Finally, one has

$$
\begin{aligned}
G_{\boldsymbol{\ell}_{n} ; \boldsymbol{k}_{N}}^{(\alpha)}= & \prod_{b=1}^{N} \prod_{\substack{a=1 \\
a \neq \alpha(b)}}^{N-1} \varpi\left(v_{a}-v_{\alpha(b)}-\mathrm{i} \frac{\Omega}{2}-\mathrm{i} \ell_{b} \omega_{1}-\mathrm{i} k_{b} \omega_{2}\right) \\
& \times \prod_{a<b}^{N}\left\{\sinh \left[\frac{\pi}{\omega_{1}}\left(v_{\alpha(a) \alpha(b)}+\mathrm{i} \ell_{a b} \omega_{1}+\mathrm{i} k_{a b} \omega_{2}\right)\right] \cdot \sinh \left[\frac{\pi}{\omega_{2}}\left(v_{\alpha(a) \alpha(b)}+\mathrm{i} \ell_{a b} \omega_{1}+\mathrm{i} k_{a b} \omega_{2}\right)\right]\right\} .
\end{aligned}
$$


Let $\alpha \in \mathcal{M}_{N}$ be given. Then, there exists $a \neq b$ such that $\left(\boldsymbol{w}^{(\alpha)}\right)_{a}=\left(\boldsymbol{w}^{(\alpha)}\right)_{b}$. A longish but straightforward calculation then shows that one has the factorisation

$$
G_{\boldsymbol{\ell}_{n} ; \boldsymbol{k}_{N}}^{(\alpha)}=(-1)^{\left(\ell_{a} k_{a}+\ell_{b} k_{b}\right)(N-2)} \cdot \sinh \left[\mathrm{i} \pi \frac{\omega_{2}}{\omega_{1}} k_{a b}\right] \cdot \sinh \left[\mathrm{i} \pi \frac{\omega_{1}}{\omega_{2}} \ell_{a b}\right] \cdot \mathcal{G}_{\boldsymbol{\ell}_{n} ; \boldsymbol{k}_{N}}^{(\alpha ; a, b)},
$$

in which $\mathcal{G}_{\boldsymbol{\ell}_{n} ; \boldsymbol{k}_{N}}^{(\alpha ;, b)}$ is symmetric under the permutation $\left(\ell_{a}, \ell_{b}\right) \hookrightarrow\left(\ell_{b}, \ell_{a}\right)$ or, independently, the permutation $\left(k_{a}, k_{b}\right) \hookrightarrow\left(k_{b}, k_{a}\right)$. It takes the explicit form

$$
\begin{aligned}
& \mathcal{G}_{\boldsymbol{\ell}_{n} ; \boldsymbol{k}_{N}}^{(\alpha ; a, b)}=(-1)^{\left(\overline{\boldsymbol{\ell}}_{N}+\overline{\boldsymbol{k}}_{N}\right)(N-3)} \prod_{\substack{c<d \\
\neq a, b}}^{N}\left\{\sinh \left[\frac{\pi}{\omega_{1}}\left(v_{\alpha(c) \alpha(d)}+\mathrm{i} k_{c d} \omega_{2}\right)\right] \cdot \sinh \left[\frac{\pi}{\omega_{2}}\left(v_{\alpha(c) \alpha(d)}+\mathrm{i} \ell_{c d} \omega_{1}\right)\right]\right\} \\
& \times \prod_{\substack{d=1 \\
\neq a, b}}^{N}\left\{\prod_{k \in\left\{k_{a}, k_{b}\right\}}\left\{\frac{\mathrm{i}}{2} \sinh \left[\frac{\pi}{\omega_{1}}\left(v_{\alpha(a) \alpha(d)}+\mathrm{i} \omega_{2}\left(k-k_{d}\right)\right)\right]\right\} \cdot \prod_{\ell \in\left\{\ell_{a}, \ell_{b}\right\}}\left\{\frac{\mathrm{i}}{2} \sinh \left[\frac{\pi}{\omega_{2}}\left(v_{\alpha(a) \alpha(d)}+\mathrm{i} \omega_{1}\left(\ell-\ell_{d}\right)\right)\right]\right\}\right\} \\
& \times \prod_{\substack{d=1 \\
\neq \alpha(a)}}^{N-1}\left\{\varpi^{2}\left(v_{d \alpha(a)}-\mathrm{i} \frac{\Omega}{2}\right)\left\{\prod_{k \in\left\{k_{a}, k_{b}\right\}} \prod_{p=1}^{k} \sinh \left[\frac{\pi}{\omega_{1}}\left(v_{d \alpha(a)}-\mathrm{i} p \omega_{2}\right)\right] \cdot \prod_{\ell \in\left\{\ell_{a}, \ell_{b}\right\}} \prod_{p=1}^{\ell} \sinh \left[\frac{\pi}{\omega_{2}}\left(v_{d \alpha(a)}-\mathrm{i} p \omega_{1}\right)\right]\right\}^{-1}\right\} \\
& \times \prod_{\substack{c=1 \\
c \neq a, b}}^{N} \prod_{\substack{d=1 \\
d \neq \alpha(c)}}^{N-1} \varpi\left(v_{d \alpha(c)}-\mathrm{i} \frac{\Omega}{2}-\mathrm{i} \ell_{c} \omega_{1}-\mathrm{i} k_{c} \omega_{2}\right)
\end{aligned}
$$

Likewise, one gets that

$$
P_{\boldsymbol{\ell}_{n} ; \boldsymbol{k}_{N}}^{(\alpha)}=(-1)^{\left(\ell_{a} k_{a}+\ell_{b} k_{b}\right)(N-1)} \cdot \boldsymbol{P}_{\boldsymbol{\ell}_{n} ; \boldsymbol{k}_{N}}^{(\alpha ; a)}
$$

where

$$
\begin{aligned}
& \mathcal{P}_{\boldsymbol{\ell}_{n} ; \boldsymbol{k}_{N}}^{(\alpha ; a, b)}=\mathrm{e}^{2 \mathrm{i} \pi x\left(\overline{\boldsymbol{v}}^{(\alpha)}+\mathrm{i} \bar{\ell}_{N} \omega_{1}+\mathrm{i} \bar{k}_{N} \omega_{2}\right)} \cdot(-2 \mathrm{i})^{\left(k_{a}+k_{b}+\ell_{a}+\ell_{b}\right)(N-1)} \cdot \prod_{\substack{c=1 \\
c \neq a, b}}^{N} \prod_{d=1}^{N-1} \varpi\left(v_{\alpha(c)}-w_{d}-\mathrm{i} \frac{\Omega}{2}-\mathrm{i} \ell_{c} \omega_{1}-\mathrm{i} k_{c} \omega_{2}\right) \\
& \times \prod_{c=1}^{N-1}\left\{\varpi^{2}\left(v_{\alpha(a)}-w_{c}-\mathrm{i} \frac{\Omega}{2}\right)\left\{\prod_{k \in\left\{k_{a}, k_{b}\right\}} \prod_{p=0}^{k-1} \sinh \left[\frac{\pi}{\omega_{1}}\left(v_{\alpha(a)}-w_{c}+\mathrm{i} p \omega_{2}\right)\right] \cdot \prod_{\ell \in\left\{\ell_{a}, \ell_{b}\right\}}^{\ell-1} \prod_{p=0} \sinh \left[\frac{\pi}{\omega_{2}}\left(v_{\alpha(a)}-w_{c}+\mathrm{i} p \omega_{1}\right)\right]\right\} .\right.
\end{aligned}
$$

Finally, one also gets $\gamma_{\boldsymbol{\ell}_{n} ; \boldsymbol{k}_{N}}^{(\alpha)}=(-1)^{\left(\ell_{a} k_{a}+\ell_{b} k_{b}\right)} \cdot \Gamma_{\boldsymbol{\ell}_{n} ; \boldsymbol{k}_{N}}^{(\alpha ; a)}$, where

$$
\Gamma_{\boldsymbol{\ell}_{n} ; \boldsymbol{k}_{N}}^{(\alpha ; a, b)}=\frac{2^{N(N-1)}}{N ! \cdot\left(\omega_{1} \omega_{2}\right)^{\frac{N}{2}-1}} \prod_{c=1}^{N}\left\{(2 \mathrm{i})^{\ell_{c}+k_{c}} \cdot \prod_{p=1}^{k_{c}} \sinh \left[\mathrm{i} p \pi \frac{\omega_{2}}{\omega_{1}}\right] \cdot \prod_{p=1}^{\ell_{c}} \sinh \left[\mathrm{i} p \pi \frac{\omega_{1}}{\omega_{2}}\right]\right\}^{-1} \cdot \prod_{\substack{c=1 \\ \neq \neq, b}}^{N}\left\{(-1)^{k_{c} \ell_{c}}\right\} .
$$

Note that $\Gamma_{\boldsymbol{\ell}_{n} ; \boldsymbol{k}_{N}}^{(\alpha ; a)}$ and $\mathcal{P}_{\boldsymbol{\ell}_{n} ; \boldsymbol{k}_{N}}^{(\alpha ; a)}$ enjoy the same symmetry properties as $\mathcal{G}_{\boldsymbol{\ell}_{n} ; \boldsymbol{k}_{N}}^{(\alpha ; a, b)}$. Thus, in the end, one has that the residue expansion of the integral reorganises as

$$
\begin{aligned}
& \operatorname{Lim}_{\boldsymbol{\varepsilon}_{N} \rightarrow \mathbf{0}^{+}}\left\{\mathscr{J}_{\boldsymbol{\varepsilon}_{N}}^{(N ; N)}\left(x \mid \boldsymbol{w}_{N-1}, \boldsymbol{v}_{N-1}\right)\right\}=\sum_{\alpha \in \mathcal{M}_{N}} \sum_{\begin{array}{c}
\ell_{c}, k_{c} \in \mathbb{N} \\
c \neq a_{\alpha}, b_{\alpha}
\end{array}} \\
& \times \sum_{\substack{\ell_{\alpha}, \ell_{b_{\alpha}} \\
\in \mathbb{N}}} \sum_{\substack{k_{\alpha}, k_{b_{\alpha}} \\
\in \mathbb{N}}}(\mathcal{G} \cdot \mathcal{P} \cdot \Gamma)_{\boldsymbol{\ell}_{n} ; \boldsymbol{k}_{N}}^{\left(\alpha ; a_{\alpha}, b_{\alpha}\right)} \cdot \sinh \left[\mathrm{i} \pi \frac{\omega_{2}}{\omega_{1}} k_{a_{\alpha} b_{\alpha}}\right] \cdot \sinh \left[\mathrm{i} \pi \frac{\omega_{1}}{\omega_{2}} \ell_{a_{\alpha} b_{\alpha}}\right] .
\end{aligned}
$$


Above, for a given $\alpha$, we denote by $a_{\alpha}, b_{\alpha}$ any two distinct integers such that $\alpha\left(a_{\alpha}\right)=\alpha\left(b_{\alpha}\right)$. Due to the symmetry properties of $\mathcal{G}, \mathcal{P}$ and $\Gamma$, the summand in (4.31) is antisymmetric under $\ell_{a_{\alpha}} \leftrightarrow \ell_{b_{\alpha}}$ as well as under $k_{a_{\alpha}} \leftrightarrow k_{b_{\alpha}}$. This entails that, in the sense of distributions, the last line in (4.31) vanishes viz.

$$
\operatorname{Lim}_{\boldsymbol{\varepsilon}_{N} \rightarrow \mathbf{0}^{+}}\left\{\mathscr{J}_{\boldsymbol{\varepsilon}_{N}}^{(N ; N)}\left(x \mid \boldsymbol{w}_{N-1}, \boldsymbol{v}_{N-1}\right)\right\}=0 .
$$

\section{- The fundamental induction equation}

Let $m \in \llbracket 0 ; N-1 \rrbracket$ and assume the $\varepsilon_{a}$ 's to be small enough. Then, in $\mathscr{J}_{\varepsilon_{N}}^{(N ; m)}$ one deforms the $y_{N}$ integration to $\mathbb{R}-\mathrm{i} \alpha$ with $\alpha>0$ small enough and such that $\alpha>\max \left\{\left|\varepsilon_{a}\right|\right\}$. One crosses the poles at

$$
y_{N}=w_{a}-\mathrm{i} \zeta_{N}^{(m)}=w_{a}-\mathrm{i} \varepsilon_{m+1}, \quad \text { with } \quad a=1, \ldots, N-1 .
$$

This yields

$$
\begin{array}{r}
\mathscr{J}_{\boldsymbol{\varepsilon}_{N}}^{(N ; m)}\left(x \mid \boldsymbol{w}_{N-1}, \boldsymbol{v}_{N-1}\right)=\int_{\mathscr{C}^{m}} \mathrm{~d}^{m} y \int_{\mathbb{R}^{N-m-1}} \prod_{a=m+1}^{N-1} \mathrm{~d} y_{a} \int_{\mathbb{R}-\mathrm{i} \alpha} \mathrm{d} y_{N} \cdot \mathcal{I}_{N}^{(m)}\left(\boldsymbol{\varepsilon}_{N} \mid x, \boldsymbol{y}_{N} ; \boldsymbol{w}_{N-1}, \boldsymbol{v}_{N-1}\right) \\
+\sum_{k=1}^{N-1} \int_{\mathscr{C}^{m}} \mathrm{~d}^{m} y \int_{\mathbb{R}^{N-m-1}} \prod_{a=m+1}^{N-1} \mathrm{~d} y_{a} \cdot \mathrm{e}^{2 \mathrm{i} \pi\left(w_{k}-\mathrm{i} \varepsilon_{m+1}\right) x} \frac{\prod_{\substack{a=1 \\
\neq k}}^{N-1} \varpi\left(w_{k a}-\mathrm{i} \frac{\Omega}{2}\right) \prod_{a=1}^{N-1} \varpi\left(v_{a}-w_{k}-\mathrm{i} \frac{\Omega}{2}+2 \mathrm{i} \varepsilon_{m+1}\right)}{\prod_{a=1}^{N-1} \varpi\left(w_{k}-y_{a}-\mathrm{i} \frac{\Omega}{2}+\mathrm{i} \zeta_{a}^{(m)}, y_{a}-w_{k}-\mathrm{i} \frac{\Omega}{2}+\mathrm{i} \zeta_{a}^{(m)}\right)} \\
\times \frac{1}{N \sqrt{\omega_{1} \omega_{2}}} \cdot \mathcal{I}_{N-1}^{(m)}\left(\boldsymbol{\varepsilon}_{N ;[m]} \mid x, \boldsymbol{y}_{N-1} ; \boldsymbol{w}_{N-1}, \boldsymbol{v}_{N-1}\right) .
\end{array}
$$

Above, the vector $\boldsymbol{\varepsilon}_{N ;[m]} \in \mathbb{R}^{N-1}$ is defined by

$$
\left(\boldsymbol{\varepsilon}_{N ;[m]}\right)_{a}=\varepsilon_{a}, a=1, \ldots, m \text { and }\left(\boldsymbol{\varepsilon}_{N ;[m]}\right)_{a}=\varepsilon_{a+1}, a=m+1, \ldots, N-1 .
$$

The fact that the last line of (4.34) does indeed take the form as written follows from the below identification

$$
\left\{\begin{array}{l}
\check{\zeta}_{a}^{(m)} \equiv \quad-\left(\boldsymbol{\varepsilon}_{N ;[m]}\right)_{a}=-\varepsilon_{a}, a=1, \ldots, m \\
\check{\zeta}_{a}^{(m)} \equiv\left(\widetilde{\varepsilon_{N ;[m]}}\right)_{a}^{(m)}=\varepsilon_{N+m+1-a}, a=m+1, \ldots, N-1 .
\end{array}\right.
$$

in which $\breve{\zeta}_{a}^{(m)}$ is the sequence built up from $\boldsymbol{\varepsilon}_{N ;[m]}$. Hence, (4.36) ensures that $\breve{\zeta}_{a}^{(m)}=\zeta_{a}^{(m)}$ for $a=1, \ldots, N-1$.

Define $\mathrm{P}_{a}$ as the operator $\left(\mathrm{P}_{a} \cdot \varepsilon_{N}\right)_{b}=(-1)^{\delta_{a b}} \boldsymbol{\varepsilon}_{b}$. Let $\bar{\zeta}_{a}^{(m)}$ be the sequence built up from the vector $\mathrm{P}_{m+1} \cdot \boldsymbol{\varepsilon}_{N}$, namely

$$
\bar{\zeta}_{a}^{(m)}=-\varepsilon_{a}, a=1, \ldots, m ; \bar{\zeta}_{N}^{(m)}=-\varepsilon_{m+1} \quad \text { and } \quad \bar{\zeta}_{a}^{(m)}=\varepsilon_{N+m+1-a}, a=m+1, \ldots, N-1 .
$$

Thus, one has

$$
\bar{\zeta}_{a}^{(m)}=\zeta_{a}^{(m+1)}, a=1, \ldots, m ; \bar{\zeta}_{N}^{(m)}=\zeta_{m+1}^{(m+1)} \quad \text { and } \quad \bar{\zeta}_{a}^{(m)}=\zeta_{a+1}^{(m+1)}, a=m+1, \ldots, N-1 .
$$

Upon defining

$$
\bar{y}_{a}=y_{a}, a=1, \ldots, m ; \bar{y}_{m+1}=y_{N} \quad \text { and } \quad \bar{y}_{a+1}=y_{a}, a=m+1, \ldots, N-1,
$$


one gets the below product identities

$$
\prod_{b=1}^{N} \varpi\left(y_{b}-w-\mathrm{i} \frac{\Omega}{2}+\mathrm{i} \bar{\zeta}_{b}^{(m)}, v-y_{b}-\mathrm{i} \frac{\Omega}{2}+\mathrm{i} \bar{\zeta}_{b}^{(m)}\right)=\prod_{b=1}^{N} \varpi\left(\bar{y}_{b}-w-\mathrm{i} \frac{\Omega}{2}+\mathrm{i} \zeta_{b}^{(m+1)}, v-\bar{y}_{b}-\mathrm{i} \frac{\Omega}{2}+\mathrm{i} \zeta_{b}^{(m+1)}\right)
$$

and

$$
\begin{aligned}
& \prod_{a<b}^{N} \varpi\left(y_{b a}-\mathrm{i} \frac{\Omega}{2}+\mathrm{i} \bar{\zeta}_{b}^{(m)}+\mathrm{i} \bar{\zeta}_{a}^{(m)}, y_{a b}-\mathrm{i} \frac{\Omega}{2}-\mathrm{i} \bar{\zeta}_{b}^{(m)}+\mathrm{i} \bar{\zeta}_{a}^{(m)}\right) \\
& =\prod_{a<b}^{N} \varpi\left(\bar{y}_{b a}-\mathrm{i} \frac{\Omega}{2}+\mathrm{i} \zeta_{b}^{(m+1)}+\mathrm{i} \zeta_{a}^{(m+1)}, \bar{y}_{a b}-\mathrm{i} \frac{\Omega}{2}-\mathrm{i} \zeta_{b}^{(m+1)}+\mathrm{i} \zeta_{a}^{(m+1)}\right) \\
& \times \prod_{a=m+2}^{N} \varpi\left(\begin{array}{l}
\bar{y}_{m+1 a}-\mathrm{i} \frac{\Omega}{2}+\mathrm{i} \zeta_{m+1}^{(m+1)}+\mathrm{i} \zeta_{a}^{(m+1)}, \bar{y}_{a m+1}-\mathrm{i} \frac{\Omega}{2}-\mathrm{i} \zeta_{m+1}^{(m+1)}+\mathrm{i} \zeta_{a}^{(m+1)} \\
\bar{y}_{m+1 a}-\mathrm{i} \frac{\Omega}{2}+\mathrm{i} \zeta_{m+1}^{(m+1)}-\mathrm{i} \zeta_{a}^{(m+1)}, \bar{y}_{a m+1}-\mathrm{i} \frac{\Omega}{2}+\mathrm{i} \zeta_{m+1}^{(m+1)}+\mathrm{i} \zeta_{a}^{(m+1)}
\end{array}\right)
\end{aligned}
$$

Thus, define

$$
\begin{aligned}
\delta \mathscr{J}_{\boldsymbol{\varepsilon}_{N}}^{(N ; m)}\left(x \mid \boldsymbol{w}_{N-1}, \boldsymbol{v}_{N-1}\right)= & \int_{\mathscr{C}^{m}} \mathrm{~d}^{m} y \int_{\mathbb{R}^{N-m-1}} \prod_{a=m+1}^{N-1} \mathrm{~d} y_{a} \int_{\mathbb{R}-\mathrm{i} \alpha} \mathrm{d} y_{N} \\
& \times\left\{\mathcal{I}_{N}^{(m)}\left(\boldsymbol{\varepsilon}_{N} \mid x, \boldsymbol{y}_{N} ; \boldsymbol{w}_{N-1}, \boldsymbol{v}_{N-1}\right)-\mathcal{I}_{N}^{(m)}\left(\mathrm{P}_{m+1} \boldsymbol{\varepsilon}_{N} \mid x, \boldsymbol{y}_{N} ; \boldsymbol{w}_{N-1}, \boldsymbol{v}_{N-1}\right)\right\},
\end{aligned}
$$

Then in the counter-term integral, viz. the one associated with $\mathrm{P}_{m+1} \boldsymbol{\varepsilon}_{N}$ in (4.42), one deforms the $y_{N}$-integration curve $\mathbb{R}-\mathrm{i} \alpha$ to $\mathscr{C}$. One picks up poles at $w_{k}+\mathrm{i} \varepsilon_{m+1}=w_{k}-\mathrm{i} \bar{\zeta}_{N}^{(m)}$ with $k=1, \ldots, N-1$, and this yields

$$
\begin{aligned}
& \int_{\mathscr{C}} \mathrm{d}^{m} y \int_{\mathbb{R}^{N-m-1}} \prod_{a=m+1}^{N-1} \mathrm{~d} y_{a} \int_{\mathbb{R}-\mathrm{i} \alpha} \mathrm{d} y_{N} \cdot \mathcal{I}_{N}^{(m)}\left(\boldsymbol{\varepsilon}_{N} \mid x, \boldsymbol{y}_{N} ; \boldsymbol{w}_{N-1}, \boldsymbol{v}_{N-1}\right) \\
& =\delta \mathscr{J}_{\boldsymbol{\varepsilon}_{N}}^{(N ; m)}\left(x \mid \boldsymbol{w}_{N-1}, \boldsymbol{v}_{N-1}\right)+\widetilde{\mathcal{J}}_{\boldsymbol{\varepsilon}_{N}}^{(N ; m+1)}\left(x \mid \boldsymbol{w}_{N-1}, \boldsymbol{v}_{N-1}\right) \\
& -\sum_{k=1}^{N-1} \int_{\mathscr{C}^{m}} \mathrm{~d}^{m} y \int_{\mathbb{R}^{N-m-1}} \prod_{a=m+1}^{N-1} \mathrm{~d} y_{a} \mathrm{e}^{2 \mathrm{i} \pi\left(w_{k}+\mathrm{i} \varepsilon_{m+1}\right) x} \cdot \frac{\prod_{\substack{a=1 \\
\neq k}}^{N-1} \varpi\left(w_{k a}-\mathrm{i} \frac{\Omega}{2}\right) \prod_{a=1}^{N-1} \varpi\left(v_{a}-w_{k}-\mathrm{i} \frac{\Omega}{2}-2 \mathrm{i} \varepsilon_{m+1}\right)}{\prod_{a=1}^{N-1} \varpi\left(w_{k}-y_{a}-\mathrm{i} \frac{\Omega}{2}+\mathrm{i} \zeta_{a}^{(m)}, y_{a}-w_{k}-\mathrm{i} \frac{\Omega}{2}+\mathrm{i} \zeta_{a}^{(m)}\right)} \\
& \times \frac{1}{N \sqrt{\omega_{1} \omega_{2}}} \cdot I_{N-1}^{(m)}\left(\boldsymbol{\varepsilon}_{N ;[m]} \mid x, \boldsymbol{y}_{N-1} ; \boldsymbol{w}_{N-1}, \boldsymbol{v}_{N-1}\right) .
\end{aligned}
$$

Above, we have introduced

$$
\begin{aligned}
\widetilde{J}_{\boldsymbol{\varepsilon}_{N}}^{(N ; m+1)}\left(x \mid \boldsymbol{w}_{N-1}, \boldsymbol{v}_{N-1}\right) & =\int_{\mathscr{C}_{m+1}} \mathrm{~d}^{m+1} y \int_{\mathbb{R}^{N-m}} \prod_{a=m+2}^{N} \mathrm{~d} y_{a} \cdot \mathcal{I}_{N}^{(m+1)}\left(\boldsymbol{\varepsilon}_{N} \mid x, \boldsymbol{y}_{N} ; \boldsymbol{w}_{N-1}, \boldsymbol{v}_{N-1}\right) \\
\times & \prod_{a=m+2}^{N} \varpi\left(\begin{array}{l}
y_{m+1}-\mathrm{i} \frac{\Omega}{2}+\mathrm{i} \zeta_{m+1}^{(m+1)}+\mathrm{i} \zeta_{a}^{(m+1)}, y_{a m+1}-\mathrm{i} \frac{\Omega}{2}-\mathrm{i} \zeta_{m+1}^{(m+1)}+\mathrm{i} \zeta_{a}^{(m+1)} \\
y_{m+1 a}-\mathrm{i} \frac{\Omega}{2}+\mathrm{i} \zeta_{m+1}^{(m+1)}-\mathrm{i} \zeta_{a}^{(m+1)}, y_{a m+1}-\mathrm{i} \frac{\Omega}{2}+\mathrm{i} \zeta_{m+1}^{(m+1)}+\mathrm{i} \zeta_{a}^{(m+1)}
\end{array}\right) .
\end{aligned}
$$


Thus, all-in-all, one gets

$$
\begin{aligned}
& \left(\mathscr{J}_{\mathcal{\varepsilon}_{N}}^{(N ; m)}-\delta \mathscr{J}_{\mathcal{E}_{N}}^{(N ; m)}-\widetilde{\mathscr{J}}_{\boldsymbol{\varepsilon}_{N}}^{(N ; m+1)}\right)\left(x \mid \boldsymbol{w}_{N-1}, \boldsymbol{v}_{N-1}\right) \\
& =\sum_{k=1}^{N-1} \frac{\mathrm{e}^{2 \mathrm{i} \pi w_{k} x}}{N \sqrt{\omega_{1} \omega_{2}}} \int_{\mathscr{C}^{m}} \mathrm{~d}^{m} y \int_{\mathbb{R}^{N-m-1}} \prod_{a=m+1}^{N-1} \mathrm{~d} y_{a} \mathcal{I}_{N-1}^{(m)}\left(\boldsymbol{\varepsilon}_{N ;[m]} \mid x, \boldsymbol{y}_{N-1} ; \boldsymbol{w}_{N-1 ;[k]}, \boldsymbol{v}_{N-1}\right) \\
& \times \frac{\prod_{\substack{a=1 \\
\neq k}}^{N-1} \varpi\left(w_{k a}-\mathrm{i} \frac{\Omega}{2}\right)}{\prod_{a=1}^{N-1} \varpi\left(w_{k}-y_{a}-\mathrm{i} \frac{\Omega}{2}+\mathrm{i} \zeta_{a}^{(m)}\right)} \cdot \mathcal{D}_{\varepsilon_{m+1}}\left(v_{N-1}, w_{k}\right)
\end{aligned}
$$

Here $\boldsymbol{w}_{N-1 ;[k]}$ is as defined through (A.1),

$$
\mathcal{D}_{\varepsilon}\left(\boldsymbol{v}_{N-1}, w_{k}\right)=\mathrm{e}^{2 \pi x \varepsilon} \prod_{a=1}^{N-1} \varpi\left(v_{a}-w_{k}-\mathrm{i} \frac{\Omega}{2}+2 \mathrm{i} \varepsilon\right)-(\varepsilon \leftrightarrow-\varepsilon) .
$$

Upon using that

$$
\varpi\left(\lambda-\mathrm{i} \frac{\Omega}{2}\right)=\frac{\mathrm{i}}{2} \frac{\varpi\left(\lambda+\mathrm{i} \frac{\tau}{2}\right)}{\sinh \left[\frac{\pi}{\omega_{1}} \lambda\right]}
$$

and applying the pole expansion

$$
\mathrm{e}^{\frac{\pi}{\omega_{1}} x \delta_{N}} \prod_{a=1}^{N-1}\left\{\frac{1}{\sinh \left[\frac{\pi}{\omega_{1}}\left(v_{a}-x\right)\right]}\right\}=\sum_{\ell=1}^{N-1} \frac{\mathrm{e}^{\frac{\pi}{\omega_{1}} v_{\ell} \delta_{N}}}{\sinh \left[\frac{\pi}{\omega_{1}}\left(v_{a}-x\right)\right]} \cdot \frac{1}{\prod_{\substack{a=1 \\
a \neq \ell}}^{N-1} \sinh \left[\frac{\pi}{\omega_{1}}\left(v_{a}-v_{\ell}\right)\right]} \quad \text { with } \quad \delta_{N}=\left\{\begin{array}{cc}
1 & N \text { odd } \\
0 & N \text { even }
\end{array}\right.
$$

one obtains the decomposition

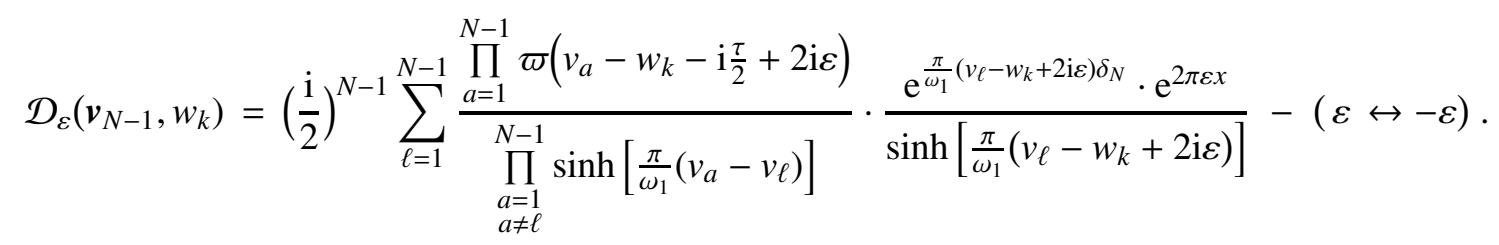

This decomposition entails that, in the sense of distributions,

$$
\mathcal{D}_{\varepsilon}\left(v_{N-1}, w_{k}\right) \underset{\varepsilon \rightarrow 0^{+}}{\longrightarrow} \sqrt{\omega_{1} \omega_{2}} \sum_{\ell=1}^{N-1} \delta\left(v_{\ell}-w_{k}\right) \prod_{\substack{a=1 \\ a \neq \ell}}^{N-1} \varpi\left(v_{a}-v_{\ell}-\mathrm{i} \frac{\Omega}{2}\right) .
$$


Thus, all-in-all

$$
\begin{aligned}
& \lim _{\varepsilon_{m+1} \rightarrow 0^{+}}\left(\mathscr{J}_{\boldsymbol{\varepsilon}_{N}}^{(N ; m)}-\delta \mathscr{J}_{\boldsymbol{\varepsilon}_{N}}^{(N ; m)}-\widetilde{\mathscr{J}}_{\boldsymbol{\varepsilon}_{N}}^{(N ; m+1)}\right)\left(x \mid \boldsymbol{w}_{N-1}, \boldsymbol{v}_{N-1}\right) \\
&=\frac{1}{N} \sum_{k, \ell=1}^{N-1} \delta\left(v_{\ell}-w_{k}\right) \cdot \mathrm{e}^{2 \mathrm{i} \pi w_{k} x} \cdot \prod_{\substack{a=1 \\
a \neq \ell}}^{N-1} \varpi\left(v_{a \ell}-\mathrm{i} \frac{\Omega}{2}\right) \\
& \times \prod_{\substack{a=1 \\
a \neq k}}^{N-1} \varpi\left(w_{k a}-\mathrm{i} \frac{\Omega}{2}\right) \mathscr{J}_{\mathcal{E}_{N ;[m]}}^{(N ; m)}\left(x \mid \boldsymbol{w}_{N-1 ;[k]}, \boldsymbol{v}_{N-1 ;[\ell]}\right) .
\end{aligned}
$$

Finally, one observes that the difference structure of the integrand of $\delta \mathscr{J}_{\mathcal{E}_{N}}^{(N ; m)}$ entails that

$$
\lim _{\boldsymbol{\varepsilon}_{N} \rightarrow \mathbf{0}^{+}}\left\{\delta \mathscr{J}_{\boldsymbol{\varepsilon}_{N}}^{(N ; m)}\left(x \mid \boldsymbol{w}_{N-1}, \boldsymbol{v}_{N-1}\right)\right\}=0 .
$$

Likewise, since the additional $\boldsymbol{\varepsilon}_{N}$ dependence present in $\widetilde{J}_{\boldsymbol{\varepsilon}_{N}}^{(N ; m+1)}\left(x \mid \boldsymbol{w}_{N-1}, \boldsymbol{v}_{N-1}\right)$ arises in the regular part of the integrand and since the singularities arising in the $\boldsymbol{\varepsilon}_{N} \rightarrow \mathbf{0}^{+}$limit of the integrand generate at most SokhotskyPlemejl distributions, one has

$$
\lim _{\boldsymbol{\varepsilon}_{N} \rightarrow \mathbf{0}^{+}}\left\{\widetilde{\mathscr{J}}_{\varepsilon_{N}}^{(N ; m+1)}\left(x \mid \boldsymbol{w}_{N-1}, \boldsymbol{v}_{N-1}\right)\right\}=\lim _{\boldsymbol{\varepsilon}_{N} \rightarrow \mathbf{0}^{+}}\left\{\mathscr{J}_{\varepsilon_{N}}^{(N ; m+1)}\left(x \mid \boldsymbol{w}_{N-1}, \boldsymbol{v}_{N-1}\right)\right\} .
$$

All of the above leads to the relation among the various integrals:

$$
\begin{aligned}
& \lim _{\boldsymbol{\varepsilon}_{N} \rightarrow \mathbf{0}^{+}}\left\{\mathscr{J}_{\boldsymbol{\varepsilon}_{N}}^{(N ; m)}\left(x \mid \boldsymbol{w}_{N-1}, \boldsymbol{v}_{N-1}\right)\right\}- \lim _{\boldsymbol{\varepsilon}_{N} \rightarrow \mathbf{0}^{+}}\left\{\mathscr{J}_{\boldsymbol{\varepsilon}_{N}}^{(N ; m+1)}\left(x \mid \boldsymbol{w}_{N-1}, \boldsymbol{v}_{N-1}\right)\right\} \\
&=\frac{1}{N} \sum_{k, \ell=1}^{N-1} \delta\left(v_{\ell}-w_{k}\right) \cdot \mathrm{e}^{2 \mathrm{i} \pi w_{k} x} \cdot \prod_{\substack{a=1 \\
a \neq \ell}}^{N-1} \varpi\left(v_{a \ell}-\mathrm{i} \frac{\boldsymbol{\Omega}}{2}\right) \\
& \times \prod_{\substack{a=1 \\
a \neq k}}^{N-1} \varpi\left(w_{k a}-\mathrm{i} \frac{\boldsymbol{\Omega}}{2}\right) \lim _{\boldsymbol{\varepsilon}_{N-1} \rightarrow \mathbf{0}^{+}}\left\{\mathscr{J}_{\left.\boldsymbol{\varepsilon}_{N-1}^{(N ; m)}\left(x \mid \boldsymbol{w}_{N-1 ;[k]}, \boldsymbol{v}_{N-1 ;[\ell]}\right)\right\},}\right.
\end{aligned}
$$

which holds for any $m \in \llbracket 0 ; N-1 \rrbracket$.

\section{- The induction sep}

Upon setting $m=N-1$ and then decreasing the value of $m$ up to $m=2$, one infers from (4.32) and (4.54) that

$$
\operatorname{Lim}_{\boldsymbol{\varepsilon}_{N} \rightarrow \boldsymbol{0}^{+}}\left\{\mathscr{J}_{\varepsilon_{N}}^{(N ; m)}\left(x \mid \boldsymbol{w}_{N-1}, \boldsymbol{v}_{N-1}\right)\right\}=0 \quad \text { for any } \quad m \in \llbracket 2 ; N \rrbracket .
$$


When $m=1$, the previous results and the induction hypothesis leads to

$$
\begin{aligned}
\operatorname{Lim}_{\boldsymbol{\varepsilon}_{N} \rightarrow \mathbf{0}^{+}}\left\{\mathscr{J}_{\boldsymbol{\varepsilon}_{N}}^{(N ; m)}\left(x \mid \boldsymbol{w}_{N-1}, \boldsymbol{v}_{N-1}\right)\right\}=\frac{1}{N(N-1)} \sum_{k, \ell=1}^{N-1} \delta\left(v_{\ell}-w_{k}\right) \cdot \mathrm{e}^{2 \mathrm{i} \pi w_{k} x} \cdot \prod_{\substack{a=1 \\
a \neq \ell}}^{N-1} \varpi\left(v_{a \ell}-\mathrm{i} \frac{\Omega}{2}\right) \\
\times \prod_{\substack{a=1 \\
a \neq k}}^{N-1} \varpi\left(w_{k a}-\mathrm{i} \frac{\Omega}{2}\right) \cdot \prod_{\substack{a \neq b \\
a, b \neq k}}^{N-1} \varpi\left(w_{a b}-\mathrm{i} \frac{\Omega}{2}\right) \cdot \delta(x) \cdot \delta_{\mathrm{sym}}\left(\boldsymbol{w}_{N-1 ;[k]}-\boldsymbol{v}_{N-1 ;[\ell]}\right) \\
=\frac{1}{N} \delta(x) \prod_{a \neq b}^{N-1} \varpi\left(w_{a b}-\mathrm{i} \frac{\Omega}{2}\right) \cdot \sum_{k=1}^{N-1} \frac{1}{N-1} \sum_{\ell=1}^{N-1} \sum_{\substack{\sigma \in \mathfrak{S}_{N-1} \\
\sigma(k)=\ell}}^{N-1} \prod_{a=1}^{N\left(v_{\sigma(a)}-w_{a}\right)} \\
=\frac{1}{N} \delta(x) \cdot \delta_{\mathrm{sym}}\left(\boldsymbol{v}_{N-1}-\boldsymbol{w}_{N-1}\right) \cdot \prod_{a \neq b}^{N-1} \varpi\left(w_{a b}-\mathrm{i} \frac{\Omega}{2}\right) .
\end{aligned}
$$

An analogous reasoning establishes the induction hypothesis for $m=0$, hence entailing the claim.

\subsection{The completeness}

We are finally in position to establish the completeness of the system of Eigenfunctions introduced in Proposition 4.1.

Proposition 4.3 The family $\left\{\psi_{\boldsymbol{y}_{N}}^{(-)}\right\}_{\boldsymbol{y}_{N} \in \mathbb{R}^{N}}$ forms a complete system on $L^{2}\left(\mathbb{R}^{N}, \mathrm{~d}^{N} x\right)$ in respect to an $L_{\mathrm{sym}}^{2}\left(\mathbb{R}^{N}, \mathrm{~d} \mu_{N}\left(\boldsymbol{y}_{N}\right)\right)$ integration of its indices, namely

$$
\frac{1}{N !} \int_{\mathbb{R}^{N}}\left(\psi_{\boldsymbol{y}_{N}}^{(-)}\left(\boldsymbol{x}_{N}^{\prime}\right)\right)^{*} \cdot \psi_{\boldsymbol{y}_{N}}^{(-)}\left(\boldsymbol{x}_{N}\right) \cdot \mathrm{d} \mu_{N}\left(\boldsymbol{y}_{N}\right)=\prod_{a=1}^{N} \delta\left(x_{a}-x_{a}^{\prime}\right)
$$

where

$$
\mathrm{d} \mu_{N}\left(\boldsymbol{y}_{N}\right)=\mu_{N}\left(\boldsymbol{y}_{N}\right) \mathrm{d}^{N} y
$$

and $\mu_{N}\left(\boldsymbol{y}_{N}\right)$ is as defined in (3.85).

The family $\left\{\psi_{y_{0}, \boldsymbol{y}_{N-1}}^{(+)}\right\}_{y_{0} \in \mathbb{R}, \boldsymbol{y}_{N-1} \in \mathbb{R}^{N-1}}$ forms a complete system on $L^{2}\left(\mathbb{R}^{N}, \mathrm{~d}^{N} x\right)$ in respect to an $L_{\cdot \times \mathrm{sym}}^{2}\left(\mathbb{R} \times \mathbb{R}^{N-1}, \mathrm{~d} y_{0} \otimes\right.$ $\left.\mathrm{d} \mu_{N-1}\left(\boldsymbol{y}_{N-1}\right)\right)$ integration of its indices, namely

$$
\frac{1}{(N-1) !} \int_{\mathbb{R}^{N}}\left(\psi_{y_{0}, \boldsymbol{y}_{N-1}}^{(+)}\left(\boldsymbol{x}_{N}^{\prime}\right)\right)^{*} \cdot \psi_{y_{0}, \boldsymbol{y}_{N-1}}^{(+)}\left(\boldsymbol{x}_{N}\right) \cdot \mathrm{d} \mu_{N}\left(\boldsymbol{y}_{N}\right)=\prod_{a=1}^{N} \delta\left(x_{a}-x_{a}^{\prime}\right) .
$$

Proof-

Assume that completeness, in the above sense, holds for $\left\{\psi_{\boldsymbol{y}_{N-1}}^{(-)}\right\}_{\boldsymbol{y}_{N-1} \in \mathbb{R}^{N-1}}$. Then, by using the Mellin-Barnes representation and the form of the regularisation one has to impose for the integral representation to make sense, 
one gets that (c.f. [19] for a rigorous treatment of the various steps in the sense of distributions)

$$
\begin{aligned}
& \frac{1}{N !} \int_{\mathbb{R}^{N}}\left(\psi_{\boldsymbol{y}_{N}}^{(-)}\left(\boldsymbol{x}_{N}^{\prime}\right)\right)^{*} \cdot \psi_{\boldsymbol{y}_{N}}^{(-)}\left(\boldsymbol{x}_{N}\right) \cdot \mathrm{d} \mu_{N}\left(\boldsymbol{y}_{N}\right) \\
& \quad=\int_{\mathbb{R}^{N-1}} \frac{\mathrm{d} \mu_{N-1}\left(\boldsymbol{w}_{N-1}\right)}{(N-1) !} \int_{\mathbb{R}^{N-1}} \frac{\mathrm{d}^{N-1} v}{(N-1) !} \cdot\left(\psi_{\boldsymbol{v}_{N-1}}^{(-)}\left(\boldsymbol{x}_{N-1}^{\prime}\right)\right)^{*} \cdot \psi_{\boldsymbol{w}_{N-1}}^{(-)}\left(\boldsymbol{x}_{N-1}\right) \cdot \lim _{\boldsymbol{\varepsilon}_{N} \rightarrow \mathbf{0}^{+}} \chi_{N}^{\left(\boldsymbol{\varepsilon}_{N}\right)}\left(\boldsymbol{w}_{N-1}, \boldsymbol{v}_{N-1} ; x_{N}, x_{N}^{\prime}\right)
\end{aligned}
$$

where

$$
\chi_{N}^{\left(\boldsymbol{\varepsilon}_{N}\right)}\left(\boldsymbol{w}_{N-1}, \boldsymbol{v}_{N-1} ; x_{N}, x_{N}^{\prime}\right)=\prod_{a=1}^{N-1}\left\{\frac{\varpi\left(w_{a}+\kappa\right)}{\varpi\left(v_{a}+\kappa\right)}\right\}^{N-1} \cdot \frac{\psi_{\overline{\boldsymbol{v}}_{N-1}}^{(-)}\left(x_{N}^{\prime}\right)}{\psi_{\overline{\boldsymbol{w}}_{N-1}^{(-)}}^{(-)}\left(x_{N}\right)} \cdot \frac{\mathcal{K}_{N}^{\left(\boldsymbol{\varepsilon}_{N}\right)}\left(\boldsymbol{w}_{N-1}, \boldsymbol{v}_{N-1} ; x_{N}-x_{N}^{\prime}\right)}{\prod_{a \neq b}^{N-1} \varpi\left(v_{a b}-\mathrm{i} \frac{\Omega}{2}\right)},
$$

while

$$
\begin{aligned}
\mathcal{K}_{N}^{\left(\boldsymbol{\varepsilon}_{N}\right)}\left(\boldsymbol{w}_{N-1}, \boldsymbol{v}_{N-1} ; x\right) & =\int_{\mathbb{R}^{N}} \frac{\mathrm{e}^{2 \mathrm{i} \pi x \bar{y}_{N}}}{N !\left(\omega_{1} \omega_{2}\right)^{N-1}} \frac{\varpi\left(\overline{\boldsymbol{y}}_{N}-\overline{\boldsymbol{v}}_{N-1}-\kappa\right)}{\varpi\left(\overline{\boldsymbol{y}}_{N}-\overline{\boldsymbol{w}}_{N-1}-\kappa\right)} \\
\times & \frac{\prod_{a=1}^{N} \prod_{b=1}^{N-1} \varpi\left(y_{a}-w_{b}-\mathrm{i} \frac{\Omega}{2}+\mathrm{i} \varepsilon_{N+1-a}\right) \varpi\left(v_{b}-y_{a}-\mathrm{i} \frac{\Omega}{2}+\mathrm{i} \varepsilon_{N+1-a}\right)}{\prod_{a<b}^{N} \varpi\left(y_{b}-y_{a}-\mathrm{i} \frac{\Omega}{2}+\mathrm{i}\left(\varepsilon_{N+1-b}+\varepsilon_{N+1-a}\right), y_{a}-y_{b}-\mathrm{i} \frac{\Omega}{2}+\mathrm{i}\left(\varepsilon_{N+1-a}-\varepsilon_{N+1-a}\right)\right)} \cdot \mathrm{d}^{N} y .
\end{aligned}
$$

The use of the integral representation,

$$
\frac{\varpi\left(\overline{\boldsymbol{y}}_{N}-\overline{\boldsymbol{v}}_{N-1}-\kappa\right)}{\varpi\left(\overline{\boldsymbol{y}}_{N}-\overline{\boldsymbol{w}}_{N-1}-\kappa\right)}=\int_{\mathbb{R}} \mathrm{d} t \int_{\mathbb{R}} \mathrm{d} s \frac{\varpi\left(s-\overline{\boldsymbol{v}}_{N-1}-\kappa\right)}{\varpi\left(s-\overline{\boldsymbol{w}}_{N-1}-\kappa\right)} \mathrm{e}^{2 \mathrm{i} \pi t\left(s-\overline{\boldsymbol{y}}_{N}\right)}
$$

allows one to recast the previous expression as

$$
\mathcal{K}_{N}^{\left(\boldsymbol{\varepsilon}_{N}\right)}\left(\boldsymbol{w}_{N-1}, \boldsymbol{v}_{N-1} ; x\right)=\int_{\mathbb{R}} \mathrm{d} t \int_{\mathbb{R}} \mathrm{d} s \frac{\varpi\left(s-\overline{\boldsymbol{v}}_{N-1}-\kappa\right)}{\varpi\left(s-\overline{\boldsymbol{w}}_{N-1}-\kappa\right)} \mathrm{e}^{2 \mathrm{i} \pi t s} \mathscr{J}_{\boldsymbol{\varepsilon}_{N}}^{(N ; 0)}\left(x-t \mid \boldsymbol{w}_{N-1}, \boldsymbol{v}_{N-1}\right),
$$

with $\mathscr{J}_{\mathcal{E}_{N}}^{(N ; 0)}$ as defined through (4.13)-(4.14). Then, by virtue of Proposition 4.2 and straightforward integrations,

$$
\lim _{\boldsymbol{\varepsilon}_{N} \rightarrow \mathbf{0}^{+}} \mathcal{K}_{N}^{\left(\boldsymbol{\varepsilon}_{N}\right)}\left(\boldsymbol{w}_{N-1}, \boldsymbol{v}_{N-1} ; x\right)=\prod_{a \neq b}^{N-1} \varpi\left(v_{a b}-\mathrm{i} \frac{\Omega}{2}\right) \cdot \delta(x) \cdot \delta_{\text {sym }}\left(\boldsymbol{w}_{N-1}-\boldsymbol{v}_{N-1}\right) .
$$

Thus, upon inserting this result in (4.61) and then (4.60), one obtains:

$$
\begin{aligned}
\frac{1}{N !} \int_{\mathbb{R}^{N}}\left(\psi_{\boldsymbol{y}_{N}}^{(-)}\left(\boldsymbol{x}_{N}^{\prime}\right)\right)^{*} \cdot \psi_{\boldsymbol{y}_{N}}^{(-)}\left(\boldsymbol{x}_{N}\right) \cdot \mathrm{d} \mu_{N}\left(\boldsymbol{y}_{N}\right) \\
=\frac{\delta\left(x_{N}-x_{N}^{\prime}\right)}{(N-1) !} \cdot \int_{\mathbb{R}^{N-1}}\left(\psi_{\boldsymbol{y}_{N-1}}^{(-)}\left(\boldsymbol{x}_{N-1}^{\prime}\right)\right)^{*} \cdot \psi_{\boldsymbol{y}_{N-1}}^{(-)}\left(\boldsymbol{x}_{N-1}\right) \cdot \mathrm{d} \mu_{N-1}\left(\boldsymbol{y}_{N-1}\right)=\prod_{a=1}^{N} \delta\left(x_{a}-x_{a}^{\prime}\right) .
\end{aligned}
$$

The proof of completeness in the case of the system $\left\{\psi_{y_{0}, y_{N-1}}^{(+)}\right\}_{y_{0} \in \mathbb{R}, \boldsymbol{y}_{N-1} \in \mathbb{R}^{N-1}}$ goes along similar lines. 


\section{Complete and orthogonal system of Eigenfunctions of $\left[\mathbf{T}_{N}(\lambda)\right]_{a b}$}

In this section we summarise the results established in the previous two sections.

Theorem 5.1 Each of the four operator entries of the monodromy matrix $T_{N}(\lambda)$ (2.3) admits a complete and orthogonal system of Eigenfunctions, $\left\{\Phi_{\boldsymbol{y}_{N}}^{(E)}\right\}_{\boldsymbol{y}_{N} \in \mathbb{R}^{N}}$ for $E \in\{A, D\}$ and $\left\{\Phi_{y_{0}, \boldsymbol{y}_{N-1}}^{(E)}\right\}_{y_{0} \in \mathbb{R}, \boldsymbol{y}_{N-1} \in \mathbb{R}^{N-1}}$, for for $E \in\{B, C\}$. Let $\mathrm{d} \mu_{N}\left(\boldsymbol{y}_{N}\right)=\mu_{N}\left(\boldsymbol{y}_{N}\right) \mathrm{d}^{N} y$ with $\mu_{N}\left(\boldsymbol{y}_{N}\right)$ as defined in (3.85).

- The system $\left\{\Phi_{\boldsymbol{y}_{N}}^{(E)}\right\}_{\boldsymbol{y}_{N} \in \mathbb{R}^{N}}, E \in\{A, D\}$, satisfies:

$$
\frac{1}{N !} \int_{\mathbb{R}^{N}}\left(\Phi_{\boldsymbol{y}_{N}}^{(E)}\left(\boldsymbol{x}_{N}^{\prime}\right)\right)^{*} \cdot \Phi_{\boldsymbol{y}_{N}}^{(E)}\left(\boldsymbol{x}_{N}\right) \cdot \mathrm{d} \mu_{N}\left(\boldsymbol{y}_{N}\right)=\prod_{a=1}^{N} \delta\left(x_{a}-x_{a}^{\prime}\right)
$$

along with

$$
\int_{\mathbb{R}^{N}}\left(\Phi_{\boldsymbol{y}_{N}^{\prime}}^{(E)}\left(\boldsymbol{x}_{N}\right)\right)^{*} \cdot \Phi_{\boldsymbol{y}_{N}}^{(E)}\left(\boldsymbol{x}_{N}\right) \cdot \mathrm{d}^{N} x=\frac{1}{\mu_{N}\left(\boldsymbol{y}_{N}\right)} \cdot \delta_{\mathrm{sym}}\left(\boldsymbol{y}_{N}-\boldsymbol{y}_{N}^{\prime}\right) .
$$

Furthermore, the generalised Eigenvalue equation takes the form

$$
E_{N}(\lambda) \cdot \Phi_{\boldsymbol{y}_{N}}^{(E)}\left(\boldsymbol{x}_{N}\right)=\prod_{a=1}^{N}\left\{-2 \mathrm{i} \sinh \left[\frac{\pi}{\omega_{1}}\left(\lambda-y_{a}\right)\right]\right\} \cdot \Phi_{\boldsymbol{y}_{N}}^{(E)}\left(\boldsymbol{x}_{N}\right) .
$$

- The system $\left\{\Phi_{y_{0}, y_{N-1}}^{(E)}\right\}_{y_{0} \in \mathbb{R}, \boldsymbol{y}_{N-1} \in \mathbb{R}^{N-1}}, E \in\{B, C\}$ satisfies:

$$
\frac{1}{(N-1) !} \int_{\mathbb{R}^{N}}\left(\Phi_{y_{0}^{\prime}, \boldsymbol{y}_{N-1}^{\prime}}^{(E)}\left(\boldsymbol{x}_{N}^{\prime}\right)\right)^{*} \cdot \Phi_{\boldsymbol{y}_{N}}^{(E)}\left(\boldsymbol{x}_{N}\right) \cdot \mathrm{d} y_{0} \cdot \mathrm{d} \mu_{N-1}\left(\boldsymbol{y}_{N-1}\right)=\prod_{a=1}^{N} \delta\left(x_{a}-x_{a}^{\prime}\right)
$$

along with

$$
\int_{\mathbb{R}^{N}}\left(\Phi_{y_{0}^{\prime}, y_{N-1}^{\prime}}^{(E)}\left(\boldsymbol{x}_{N}\right)\right)^{*} \cdot \Phi_{y_{0}, \boldsymbol{y}_{N-1}}^{(E)}\left(\boldsymbol{x}_{N}\right) \cdot \mathrm{d}^{N} x=\frac{1}{\mu_{N-1}\left(\boldsymbol{y}_{N-1}\right)} \cdot \delta\left(y_{0}-y_{0}^{\prime}\right) \cdot \delta_{\mathrm{sym}}\left(\boldsymbol{y}_{N-1}-\boldsymbol{y}_{N-1}^{\prime}\right) .
$$

Finally, the generalised Eigenvalue equation takes the form

$$
E_{N}(\lambda) \cdot \Phi_{y_{0}, y_{N-1}}^{(E)}\left(\boldsymbol{x}_{N}\right)=\mathrm{e}^{2 \pi \omega_{2} y_{0}} \prod_{a=1}^{N-1}\left\{-2 \mathrm{i} \sinh \left[\frac{\pi}{\omega_{1}}\left(\lambda-y_{a}\right)\right]\right\} \cdot \Phi_{y_{0}, \boldsymbol{y}_{N-1}}^{(E)}\left(\boldsymbol{x}_{N}\right) .
$$

The generalised Eigenfunctions admit Gauss-Givental and Mellin-Barnes integral representations:

$$
\Phi_{\boldsymbol{y}_{N}}^{(\boldsymbol{A})}\left(\boldsymbol{x}_{N}\right)=\psi_{\boldsymbol{y}_{N}}^{(-)}\left(\boldsymbol{x}_{N}\right)=c_{A} \varphi_{\boldsymbol{y}_{N}}^{(-)}\left(\boldsymbol{x}_{N}\right) \quad \text { and } \quad \Phi_{y_{0}, \boldsymbol{y}_{N-1}}^{(B)}\left(\boldsymbol{x}_{N}\right)=\psi_{y_{0}, \boldsymbol{y}_{N-1}}^{(+)}\left(\boldsymbol{x}_{N}\right)=c_{B} \varphi_{y_{0}, \boldsymbol{y}_{N-1}}^{(+)}\left(\boldsymbol{x}_{N}\right)
$$

Above, $c_{A}$, resp. $c_{B}$, are $\boldsymbol{y}_{N}$, resp. $y_{0}, \boldsymbol{y}_{N-1}$, independent constants equal to \pm 1 . 
We conjecture that, in fact, the proportionality constants in (5.7) equal 1.

Proof-

We shall only establish the properties of the generalised Eigenfunctions of the operator $A_{N}(\lambda)$, as the case of the $\mathrm{B}_{N}(\lambda)$ operator can be dealt with similarly. Furthermore, the case of the operators $\mathrm{D}_{N}(\lambda)$ and $\mathrm{C}_{N}(\lambda)$ is a direct consequence of the results relative to the operators $\mathrm{A}_{N}(\lambda)$ and $\mathrm{B}_{N}(\lambda)$.

Indeed, observe that upon introducing the operator $\Omega_{a}$ such that

$$
\Omega_{a} \mathrm{x}_{a} \Omega_{a}=-\mathrm{x}_{a} \quad \text { and } \quad \Omega_{a} \mathrm{p}_{a} \Omega_{a}=-\mathrm{p}_{a},
$$

one has the relation

$$
\Omega_{n} \cdot \mathrm{L}_{n}^{(\kappa)}(\lambda) \cdot \Omega_{n}=\sigma^{x} \cdot \mathrm{L}_{n}^{(\kappa)}(\lambda) \cdot \sigma^{x} \quad \text { i.e. } \quad \prod_{a=1}^{N} \Omega_{a} \cdot \mathrm{T}_{N}(\lambda) \cdot \prod_{a=1}^{N} \Omega_{a}=\sigma^{x} \cdot \mathrm{T}_{N}(\lambda) \cdot \sigma^{x} .
$$

This entails that

$$
\prod_{a=1}^{N} \Omega_{a} \cdot \mathrm{D}_{N}(\lambda) \cdot \prod_{a=1}^{N} \Omega_{a}=\mathrm{A}_{N}(\lambda) \quad \text { and } \quad \prod_{a=1}^{N} \Omega_{a} \cdot \mathrm{C}_{N}(\lambda) \cdot \prod_{a=1}^{N} \Omega_{a}=\mathrm{B}_{N}(\lambda) .
$$

Thus, if $\left\{\Phi_{\boldsymbol{y}_{N}}^{(\mathrm{A})}\right\}_{\boldsymbol{y}_{N} \in \mathbb{R}^{N}}$, resp. $\left\{\Phi_{y_{0}, \boldsymbol{y}_{N-1}}^{(\mathrm{B})}\right\}_{y_{0} \in \mathbb{R}, \boldsymbol{y}_{N-1} \in \mathbb{R}^{N-1}}$, is the complete and orthogonal system of generalised Eigenfunctions of the $\mathrm{A}_{N}(\lambda)$, resp. $\mathrm{B}_{N}(\lambda)$, operator then

$$
\left\{\prod_{a=1}^{N} \Omega_{a} \cdot \Phi_{\boldsymbol{y}_{N}}^{(\mathrm{A})}\right\}_{\boldsymbol{y}_{N} \in \mathbb{R}^{N}}, \quad \text { resp. } \quad\left\{\prod_{a=1}^{N} \Omega_{a} \cdot \Phi_{y_{0}, \boldsymbol{y}_{N-1}}^{(\mathrm{B})}\right\}_{y_{0} \in \mathbb{R}, \boldsymbol{y}_{N-1} \in \mathbb{R}^{N-1}},
$$

is the complete and orthogonal system of generalised Eigenfunctions of the $\mathrm{D}_{N}(\lambda)$, resp. $\mathrm{C}_{N}(\lambda)$, operator. Furthermore, the form of the generalised Eigenvalues is a direct consequence of the conjugation relation between the operators (5.10) and the form of the generalised Eigenvalues of the operators $\mathrm{A}_{N}(\lambda)$ and $\mathrm{B}_{N}(\lambda)$.

\section{- Proprotionality of $\varphi_{\boldsymbol{y}_{N}}^{(-)}$and $\psi_{\boldsymbol{y}_{N}}^{(-)}$}

We first show that, for any fixed $\boldsymbol{y}_{N} \in \mathbb{R}^{N}$, the functions $\varphi_{\boldsymbol{y}_{N}}^{(-)}$and $\psi_{\boldsymbol{y}_{N}}^{(-)}$are are non-identically zero. Given $f \in$ $L_{\text {sym }}^{2}\left(\mathbb{R}^{N}, \mathrm{~d} \mu_{N}\left(\boldsymbol{y}_{N}\right)\right)$, smooth and compactly supported, define

$$
\mathcal{U}_{N}[f]\left(\boldsymbol{x}_{N}\right)=\frac{1}{N !} \int_{\mathbb{R}^{N}} \varphi_{\boldsymbol{y}_{N}^{(-)}}\left(\boldsymbol{x}_{N}\right) f\left(\boldsymbol{y}_{N}\right) \mathrm{d} \mu_{N}\left(\boldsymbol{y}_{N}\right) .
$$

Then, the orthogonality relation satisfied by the functions $\varphi_{\boldsymbol{y}_{N}}^{(-)}\left(\boldsymbol{x}_{N}\right)$ ensures that, for any such $f$, it holds

$$
\int_{\mathbb{R}^{N}} \mathrm{~d}^{N} x\left(\varphi_{\boldsymbol{y}_{N}}^{(-)}\left(\boldsymbol{x}_{N}\right)\right)^{*} \cdot \mathcal{U}_{N}[f]\left(\boldsymbol{x}_{N}\right)=f\left(\boldsymbol{y}_{N}\right) .
$$

Thus, $\varphi_{\boldsymbol{y}_{N}}^{(-)}$cannot be identically zero.

Regarding to $\psi_{\boldsymbol{y}_{N}}^{(-)}$, as in [18], one may compute the $\boldsymbol{x}_{N} \rightarrow \infty, x_{a+1}-x_{a} \rightarrow+\infty$ of $\psi_{\boldsymbol{y}_{N}}^{(-)}\left(\boldsymbol{x}_{N}\right)$ staring from its Mellin-Barnes integral representation by pushing the various integration contours slightly in the upper-half plane. This shows that the function is non-vanishing in this asymptotic regime, and hence is non-identically zero.

Since 
- $\mathrm{A}_{N}(\lambda) \psi_{\boldsymbol{y}_{N}}^{(-)}=a\left(\lambda \mid \boldsymbol{y}_{N}\right) \psi_{\boldsymbol{y}_{N}}^{(-)}$with $a\left(\lambda \mid \boldsymbol{y}_{N}\right)=\prod_{a=1}^{N}\left\{-2 \mathrm{i} \sinh \left[\frac{\pi}{\omega_{1}}\left(\lambda-y_{a}\right)\right]\right\}$

- $\psi_{\boldsymbol{y}_{N}}^{(-)}$is non-identically zero,

- the system $\left\{\psi_{\boldsymbol{y}_{N}}^{(-)}\right\}_{\boldsymbol{y}_{N} \in \mathbb{R}^{N}}$ forms a complete system,

each generalised Eigenvalue $a\left(\lambda \mid \boldsymbol{y}_{N}\right)$ of $\mathrm{A}_{N}(\lambda)$ appears with exactly multiplicity 1 . Since it also holds $\mathrm{A}_{N}(\lambda) \varphi_{\boldsymbol{y}_{N}}^{(-)}=$ $a\left(\lambda \mid \boldsymbol{y}_{N}\right) \varphi_{\boldsymbol{y}_{N}}^{(-)}$, with $\varphi_{\boldsymbol{y}_{N}}^{(-)}$non-identically zero, it follows that there exists a constant $c_{N}\left(\boldsymbol{y}_{N}, \kappa\right) \in \mathbb{C}^{*}$ such that

$$
\psi_{\boldsymbol{y}_{N}}^{(-)}=c_{N}\left(\boldsymbol{y}_{N}, \kappa\right) \varphi_{\boldsymbol{y}_{N}}^{(-)} .
$$

The proprotionality constant may, in principle, depend on $\boldsymbol{y}_{N}$ and the representation parameter $\kappa$. Below, we establish various properties that ought to be satisfied by these constants. This will strongly constrain its value.

\section{- $c_{N}\left(\boldsymbol{y}_{N}, \kappa\right)$ is unimodular}

We first establish that the proportionality constant is unimodular. The orthogonality relation for $\varphi_{\boldsymbol{y}_{N}}^{(-)}$leads to the relation

$$
\int_{\mathbb{R}^{N}}\left(\psi_{\boldsymbol{y}_{N}^{\prime}}^{(-)}\left(\boldsymbol{x}_{N}\right)\right)^{*} \cdot \psi_{\boldsymbol{y}_{N}}^{(-)}\left(\boldsymbol{x}_{N}\right) \cdot \mathrm{d}^{N} x=\frac{\left|c_{N}\left(\boldsymbol{y}_{N}, \kappa\right)\right|^{2}}{\mu_{N}\left(\boldsymbol{y}_{N}\right)} \cdot \delta_{\mathrm{sym}}\left(\boldsymbol{y}_{N}-\boldsymbol{y}_{N}^{\prime}\right)
$$

Integrating both sides of this equation versus

$$
\frac{\mathrm{d} \mu_{N}\left(\boldsymbol{y}_{N}^{\prime}\right)}{N !} \otimes \frac{\mathrm{d} \mu_{N}\left(\boldsymbol{y}_{N}\right)}{N !} \psi_{\boldsymbol{y}_{N}^{\prime}}^{(-)}\left(\boldsymbol{x}_{N}^{\prime}\right)\left(\psi_{\boldsymbol{y}_{N}}^{(-)}\left(\boldsymbol{x}_{N}^{\prime \prime}\right)\right)^{*}
$$

and using completeness of the $\psi_{\boldsymbol{y}_{N}^{\prime}}^{(-)}$one is lead to

$$
\prod_{a=1}^{N} \delta\left(x_{a}^{\prime}-x_{a}^{\prime \prime}\right)=\left|c_{N}\left(\boldsymbol{y}_{N}, \kappa\right)\right|^{2} \prod_{a=1}^{N} \delta\left(x_{a}^{\prime}-x_{a}^{\prime \prime}\right) .
$$

Thus, indeed, one has that $\left|c_{N}\left(\boldsymbol{y}_{N}, \kappa\right)\right|=1$.

\section{- $c_{N}\left(y_{N}, \kappa\right)$ is invariant under $\kappa$-reflections}

It follows from (2.10) that

$$
\prod_{a=1}^{N} D_{-\kappa}\left(\mathrm{p}_{a}\right) \cdot \mathrm{T}_{N}(\lambda ; \kappa)=\mathrm{T}_{N}(\lambda ;-\kappa) \cdot \prod_{a=1}^{N} D_{-\kappa}\left(\mathrm{p}_{a}\right)
$$

where we have explicitly stressed the dependence of the monodromy matrix on the parameter $\kappa$. In the following, it will be convenient to make also explicit the dependence of the functions $\psi_{\boldsymbol{y}_{N}}^{(-)}, \varphi_{\boldsymbol{y}_{N}}^{(-)}$on $\kappa, v i z . \psi_{\boldsymbol{y}_{N}}^{(-)}\left(\boldsymbol{x}_{N} ; \kappa\right), \varphi_{\boldsymbol{y}_{N}}^{(-)}\left(\boldsymbol{x}_{N} ; \kappa\right)$. The intertwining of the monodromy matrix suggests that analogous relations should exist between the generalised Eigenfunctions. These will be established below.

The Mellin-Barnes integral representation induction immediately leads to

$$
\psi_{\boldsymbol{y}_{N}}^{(-)}\left(\boldsymbol{x}_{N} ; \kappa\right)=\prod_{s=1}^{N-1}\left\{\int_{\left(\mathbb{R}-\mathrm{i} \alpha_{s}\right)^{N-s}} \frac{\mathrm{d}^{N-s} w^{(s)}}{(N-s) !}\right\} \prod_{s=1}^{N} \psi_{\overline{\boldsymbol{w}}_{N-s+1}^{(s-1)}-\overline{\boldsymbol{w}}_{N-s}^{(s)}}^{(-)}\left(x_{N-s+1}\right) \prod_{s=1}^{N}\left\{\Phi\left(\boldsymbol{w}_{N-s}^{(s)} \mid \boldsymbol{w}_{N-s+1}^{(s-1)}\right)\right\}
$$


which can be reorganised as

$$
\psi_{\boldsymbol{y}_{N}}^{(-)}\left(\boldsymbol{x}_{N} ; \kappa\right)=\sigma\left(\boldsymbol{y}_{N} ; \kappa\right) \prod_{s=1}^{N-1}\left\{\int_{\left(\mathbb{R}-\mathrm{i} \alpha_{s}\right)^{N-s}} \frac{\mathrm{d}^{N-s} w^{(s)}}{(N-s) !}\right\} \prod_{s=1}^{N} \psi_{\overline{\boldsymbol{w}}_{N-s+1}^{(s-1)}-\overline{\boldsymbol{w}}_{N-s}^{(s)}}^{(-)}\left(x_{N-s+1}\right) \cdot \xi_{\kappa}\left(\left\{\boldsymbol{w}_{N-s}^{(s)}\right\}_{s=1}^{N}\right)
$$

where

$$
\xi_{k}\left(\left\{\boldsymbol{w}_{N-s}^{(s)}\right\}_{s=1}^{N}\right)=\frac{\prod_{s=1}^{N-1} \prod_{a=1}^{N-s} \varpi\left(w_{a}^{(s)}-\kappa, w_{a}^{(s)}+\kappa\right)}{\prod_{s=1}^{N} \varpi\left(\overline{\boldsymbol{w}}_{N-s+1}^{(s-1)}-\overline{\boldsymbol{w}}_{N-s}^{(s)}-\kappa\right)} \cdot \prod_{s=1}^{N-1}\left\{\frac{\prod_{a=1}^{N-s} \prod_{b=1}^{N-s+1} \varpi\left(w_{b}^{(s-1)}-w_{a}^{(s)}-\mathrm{i} \frac{\Omega}{2}\right)}{\left(\omega_{1} \omega_{2}\right)^{N-s} \cdot \prod_{a \neq b}^{N-s} \varpi\left(w_{b}^{(s)}-w_{a}^{(s)}-\mathrm{i} \frac{\Omega}{2}\right)}\right\},
$$

and

$$
\sigma\left(\boldsymbol{y}_{N} ; \kappa\right)=\prod_{a=1}^{N} \frac{\varpi\left(y_{a}-\kappa\right)}{\left[\varpi\left(y_{a}+\kappa\right)\right]^{N-1}} .
$$

The relation

$$
\left(D_{-\kappa}(\mathrm{p}) \cdot \psi_{y}^{(-)}\right)(x)=D_{-\kappa}(y) \psi_{y}^{(-)}(x)
$$

and the fact that

$$
\xi_{\kappa}\left(\left\{\boldsymbol{w}_{N-s}^{(s)}\right\}_{s=1}^{N}\right)=\prod_{s=1}^{N} \frac{\varpi\left(\overline{\boldsymbol{w}}_{N-s+1}^{(s-1)}-\overline{\boldsymbol{w}}_{N-s}^{(s)}+\kappa\right)}{\varpi\left(\overline{\boldsymbol{w}}_{N-s+1}^{(s-1)}-\overline{\boldsymbol{w}}_{N-s}^{(s)}-\kappa\right)} \cdot \xi_{-\kappa}\left(\left\{\boldsymbol{w}_{N-s}^{(s)}\right\}_{s=1}^{N}\right)
$$

then immediately leads to

$$
\prod_{a=1}^{N} D_{-\kappa}\left(\mathrm{p}_{a}\right) \cdot \psi_{\boldsymbol{y}_{N}}^{(-)}\left(\boldsymbol{x}_{N} ; \kappa\right)=\prod_{a=1}^{N} D_{-\kappa}^{N}\left(y_{a}\right) \cdot \psi_{\boldsymbol{y}_{N}}^{(-)}\left(\boldsymbol{x}_{N} ;-\kappa\right)
$$

The same property holds for $\varphi_{\boldsymbol{y}_{N}}^{(-)}$, namely

$$
\prod_{a=1}^{N} D_{-\kappa}\left(\mathrm{p}_{a}\right) \cdot \varphi_{\boldsymbol{y}_{N}}^{(-)}\left(\boldsymbol{x}_{N} ; \kappa\right)=\prod_{a=1}^{N} D_{-\kappa}^{N}\left(y_{a}\right) \cdot \varphi_{\boldsymbol{y}_{N}}^{(-)}\left(\boldsymbol{x}_{N} ;-\kappa\right) .
$$

Indeed, this identity is a direct consequence of an inductive application of Lemma 3.2 on the level of the recursive construction of $\varphi_{\boldsymbol{y}_{N}}^{(-)}\left(\boldsymbol{x}_{N} ; \kappa\right)$.

This entails that $c_{N}\left(\boldsymbol{y}_{N}, \kappa\right)=c_{N}\left(\boldsymbol{y}_{N},-\kappa\right)$.

\section{- Complex conjugation of $c_{N}\left(\boldsymbol{y}_{N}, \kappa\right)$}

Finally, the $\Lambda_{y, \epsilon}^{(N)}$ operator can be represented as

$$
\left(\Lambda_{y, \epsilon}^{(N)} \cdot f\right)\left(\boldsymbol{x}_{N}\right)=\int_{\mathbb{R}^{N-1}} \Lambda_{y, \epsilon}^{(N)}\left(\boldsymbol{x}_{N}, \boldsymbol{x}_{N-1}^{\prime} ; \kappa\right) f\left(\boldsymbol{x}_{N-1}^{\prime}\right) \cdot \mathrm{d}^{N-1} x^{\prime}
$$


where the integral kernel takes the form

$$
\begin{aligned}
\Lambda_{y, \epsilon}^{(N)}\left(\boldsymbol{x}_{N}, \boldsymbol{x}_{N-1}^{\prime} ; \kappa\right)= & \frac{\mathrm{e}^{2 \mathrm{i} \pi y_{-} x_{1}} \mathrm{e}^{2 \mathrm{i} \pi y_{+}^{\star} x_{N}}}{\left(\sqrt{\omega_{1} \omega_{2}} \mathcal{A}\left(y_{+}\right)\right)^{N-1}} \\
& \quad \times \prod_{a=1}^{N-1}\left\{D_{y_{-}-y_{+}}\left(\omega_{1} \omega_{2} x_{a a+1}\right) D_{y_{-}^{\star}}\left(\omega_{1} \omega_{2}\left(x_{a}-x_{a}^{\prime}\right)\right) D_{y_{+}}\left(\omega_{1} \omega_{2}\left(x_{a+1}-x_{a}^{\prime}\right)\right)\right\} .
\end{aligned}
$$

It is then immediate to check that

$$
\left(\Lambda_{y, \epsilon}^{(N)}\left(\boldsymbol{x}_{N}, \boldsymbol{x}_{N-1}^{\prime} ; \kappa\right)\right)^{*}=\Lambda_{-y, \epsilon}^{(N)}\left(\boldsymbol{x}_{N}, \boldsymbol{x}_{N-1}^{\prime} ;-\kappa\right) .
$$

This relation then leads to

$$
\left(\varphi_{\boldsymbol{y}_{N}}^{(-)}\left(\boldsymbol{x}_{N} ; \kappa\right)\right)^{*}=\varphi_{-\boldsymbol{y}_{N}}^{(-)}\left(\boldsymbol{x}_{N} ;-\kappa\right) .
$$

Now starting from the Mellin-Barnes representation (5.20) and upon using that for

$$
\boldsymbol{w}_{N-s}^{(s)} \in \mathbb{R}^{N-s} \quad \text { and } \quad \boldsymbol{\alpha}_{s}=\alpha_{s}(1, \ldots, 1) \in \mathbb{R}^{N-s} \quad \text { with } \quad \alpha_{s} \text { real }
$$

it holds

$$
\left(\xi_{k}\left(\left\{-\boldsymbol{w}_{N-s}^{(s)}-\mathrm{i} \boldsymbol{\alpha}_{s}\right\}_{s=1}^{N}\right)\right)^{*}=\xi_{-\kappa}\left(\left\{\boldsymbol{w}_{N-s}^{(s)}-\mathrm{i} \boldsymbol{\alpha}_{s}\right\}_{s=1}^{N}\right),
$$

one entails that, as well

$$
\left(\psi_{\boldsymbol{y}_{N}}^{(-)}\left(\boldsymbol{x}_{N} ; \kappa\right)\right)^{*}=\psi_{-\boldsymbol{y}_{N}}^{(-)}\left(\boldsymbol{x}_{N} ;-\kappa\right) .
$$

This entails that $\left(c_{N}\left(\boldsymbol{y}_{N}, \kappa\right)\right)^{*}=c_{N}\left(-\boldsymbol{y}_{N},-\kappa\right)$. Hence, by invoking the $\kappa$-reflection property, one infers that the constant $c_{N}$ behaves under complex conjugation as

$$
\left(c_{N}\left(\boldsymbol{y}_{N}, \kappa\right)\right)^{*}=c_{N}\left(-\boldsymbol{y}_{N}, \kappa\right) .
$$

\section{- $c_{N}\left(\boldsymbol{y}_{N}, \kappa\right)$ is $\boldsymbol{y}_{N}$-independent}

It remains to prove that the constant does not depend on $\boldsymbol{y}_{N}$. As follows from Propositions 3.3 and 3.2, $\varphi_{\boldsymbol{y}_{N}}^{(-)}$ satisfies identically the same equations (4.6)-(4.7) as $\psi_{\boldsymbol{y}_{N}}^{(-)}$. By projecting these on a given variable $\boldsymbol{y}_{N}$, this yields that

$$
c_{N}\left(\boldsymbol{y}_{N}, \kappa\right)=c_{N}\left(\boldsymbol{y}_{N}+\mathrm{i} \omega_{2} \boldsymbol{e}_{k}, \kappa\right)
$$

where $\boldsymbol{e}_{k}$ is the $k^{\text {th }}$ unit vector in $\mathbb{R}^{N}$. The same equation holds for $\omega_{1} \leftrightarrow \omega_{2}$ since both $\varphi_{\boldsymbol{y}_{N}}^{(-)}$and $\psi_{\boldsymbol{y}_{N}}^{(-)}$satisfy the dual equations to (4.6)-(4.7) as well.

Thus, all-in-all, we get that $\left(c_{N}\left(\boldsymbol{y}_{N}, \kappa\right)\right)^{*}=c_{N}\left(\boldsymbol{y}_{N}, \kappa\right)$, what along with $\left|c_{N}\left(\boldsymbol{y}_{N}, \kappa\right)\right|=1$ implies that $c_{N}\left(\boldsymbol{y}_{N}, \kappa\right)=$ \pm 1 . 


\section{The Sinh-Gordon model}

The Lax matrix of the lattice Sinh-Gordon model takes the form [2]:

$$
\mathrm{L}_{n}^{(\mathrm{SG})}(\lambda)=\sigma^{x} \mathrm{~L}_{n}^{(\kappa)}(\lambda) .
$$

The monodromy matrix of the $N$-site lattice Sinh-Gordon model thus takes the form

$$
\mathrm{T}_{N}^{(\mathrm{SG})}(\lambda)=\mathrm{L}_{1}^{(\mathrm{SG})}(\lambda) \cdots \mathrm{L}_{N}^{(\mathrm{SG})}(\lambda)=\left(\begin{array}{ll}
\mathrm{A}_{N}^{(\mathrm{SG})}(\lambda) & \mathrm{B}_{N}^{(\mathrm{SG})}(\lambda) \\
\mathrm{C}_{N}^{(\mathrm{SG})}(\lambda) & \mathrm{D}_{N}^{(\mathrm{SG})}(\lambda)
\end{array}\right) .
$$

The analysis of the system of Eigenfunctions of the operators $\mathrm{A}_{N}$ and $\mathrm{B}_{N}$ that was carried out in the previous sections allows us to access to the system of Eigenfunctions of the operator $\mathrm{B}_{N}^{(\mathrm{SG})}(\lambda)$ and characterise its spectrum. In this manner we prove the conjecture raised in [2] relatively to the spectrum of this operator. In order to state the result, it is convenient to recall the operators $\Omega_{a}$ introduced in (5.9) which enjoy the exchange relations:

$$
\Omega_{a} \mathrm{x}_{a} \Omega_{a}=-\mathrm{x}_{a} \quad \text { and } \quad \Omega_{a} \mathrm{p}_{a} \Omega_{a}=-\mathrm{p}_{a} .
$$

Theorem 6.1 The operator $B_{N}^{(\mathrm{SG})}(\lambda)$ admits a complete and orthogonal system of Eigenfunctions:

- for $N$ odd, this systems is $\left\{\Phi_{\boldsymbol{y}_{N}}\right\}_{\boldsymbol{y}_{N} \in \mathbb{R}^{N}}$ and it satisfies :

$$
\frac{1}{N !} \int_{\mathbb{R}^{N}}\left(\Phi_{\boldsymbol{y}_{N}}\left(\boldsymbol{x}_{N}^{\prime}\right)\right)^{*} \cdot \Phi_{\boldsymbol{y}_{N}}\left(\boldsymbol{x}_{N}\right) \cdot \mathrm{d} \mu_{N}\left(\boldsymbol{y}_{N}\right)=\prod_{a=1}^{N} \delta\left(x_{a}-x_{a}^{\prime}\right)
$$

along with

$$
\int_{\mathbb{R}^{N}}\left(\Phi_{\boldsymbol{y}_{N}^{\prime}}\left(\boldsymbol{x}_{N}\right)\right)^{*} \cdot \Phi_{\boldsymbol{y}_{N}}\left(\boldsymbol{x}_{N}\right) \cdot \mathrm{d}^{N} x=\frac{1}{\mu_{N}\left(\boldsymbol{y}_{N}\right)} \cdot \delta_{\mathrm{sym}}\left(\boldsymbol{y}_{N}-\boldsymbol{y}_{N}^{\prime}\right) .
$$

Furthermore, the generalised Eigenvalue equation takes the form

$$
B_{N}^{(\mathrm{SG})}(\lambda) \cdot \Phi_{\boldsymbol{y}_{N}}\left(\boldsymbol{x}_{N}\right)=\prod_{a=1}^{N}\left\{-2 \mathrm{i} \sinh \left[\frac{\pi}{\omega_{1}}\left(\lambda-y_{a}\right)\right]\right\} \cdot \Phi_{\boldsymbol{y}_{N}}\left(\boldsymbol{x}_{N}\right) .
$$

- for $N$ even, this systems is $\left\{\Phi_{y_{0}, \boldsymbol{y}_{N-1}}\right\}_{y_{0} \in \mathbb{R}, \boldsymbol{y}_{N-1} \in \mathbb{R}^{N-1}}$ and it satisfies :

$$
\frac{1}{(N-1) !} \int_{\mathbb{R}^{N}}\left(\Phi_{y_{0}^{\prime}, \boldsymbol{y}_{N-1}^{\prime}}\left(\boldsymbol{x}_{N}^{\prime}\right)\right)^{*} \cdot \Phi_{\boldsymbol{y}_{N}}\left(\boldsymbol{x}_{N}\right) \cdot \mathrm{d} y_{0} \cdot \mathrm{d} \mu_{N-1}\left(\boldsymbol{y}_{N-1}\right)=\prod_{a=1}^{N} \delta\left(x_{a}-x_{a}^{\prime}\right)
$$

along with

$$
\int_{\mathbb{R}^{N}}\left(\Phi_{y_{0}^{\prime}, \boldsymbol{y}_{N-1}^{\prime}}\left(\boldsymbol{x}_{N}\right)\right)^{*} \cdot \Phi_{y_{0}, \boldsymbol{y}_{N-1}}\left(\boldsymbol{x}_{N}\right) \cdot \mathrm{d}^{N} x=\frac{1}{\mu_{N-1}\left(\boldsymbol{y}_{N-1}\right)} \cdot \delta\left(y_{0}-y_{0}^{\prime}\right) \cdot \delta_{\mathrm{sym}}\left(\boldsymbol{y}_{N-1}-\boldsymbol{y}_{N-1}^{\prime}\right) .
$$

Finally, the generalised Eigenvalue equation takes the form

$$
B_{N}^{(\mathrm{SG})}(\lambda) \cdot \Phi_{y_{0}, \boldsymbol{y}_{N-1}}\left(\boldsymbol{x}_{N}\right)=\mathrm{e}^{2 \pi \omega_{2} y_{0}} \prod_{a=1}^{N-1}\left\{-2 \mathrm{i} \sinh \left[\frac{\pi}{\omega_{1}}\left(\lambda-y_{a}\right)\right]\right\} \cdot \Phi_{y_{0}, \boldsymbol{y}_{N-1}}\left(\boldsymbol{x}_{N}\right) .
$$


Proof-

The relation between the Lax matrices (6.1) implies that the monodromy matrix of the Sinh-Gordon model is related to the one of the modular XXZ chain as

$$
\mathrm{T}_{N}^{(\mathrm{SG})}(\lambda)=\left\{\begin{array}{cl}
\prod_{a=1}^{N / 2} \Omega_{2 a-1} \cdot \mathrm{T}_{N}(\lambda) \cdot \prod_{a=1}^{N / 2} \Omega_{2 a-1} & \text { if } N \text { is even } \\
\prod_{a=1}^{(N+1) / 2} \Omega_{2 a-1} \cdot \mathrm{T}_{N}(\lambda) \sigma^{x} \cdot \prod_{a=1}^{(N+1) / 2} \Omega_{2 a-1} & \text { if } N \text { is odd }
\end{array}\right.
$$

Indeed, this relation is a simple consequence of the local identity

$$
\sigma^{x} \mathrm{~L}_{n}^{(\kappa)}(\lambda) \sigma^{x}=\Omega_{n} \mathrm{~L}_{n}^{(\kappa)}(\lambda) \Omega_{n}
$$

The representation (6.10) then ensures that the system of generalised Eigenfunctions of the operator $\mathrm{B}_{N}^{(\mathrm{SG})}(\lambda)$ is given by

$$
\left\{\begin{array}{cl}
\prod_{a=1}^{N / 2} \Omega_{2 a-1} \cdot \Phi_{y_{N}}^{(\mathrm{A})} & \text { if } N \text { is even } \\
\prod_{a=1}^{(N+1) / 2} \Omega_{2 a-1} \cdot \Phi_{y_{0}, \boldsymbol{y}_{N-1}}^{(\mathrm{B})} & \text { if } N \text { is odd }
\end{array},\right.
$$

where $\Phi_{y_{N}}^{(\mathrm{A})}, \Phi_{y_{0}, y_{N-1}}^{(\mathrm{B})}$ are as introduced in Theorem 5.1. The rest follows from the stated properties of the functions $\Phi_{y_{N}}^{(\mathrm{A})}, \Phi_{y_{0}, y_{N-1}}^{(\mathrm{B})}$ in that theorem.

\section{Conclusion}

In this work we have constructed the Eigenfunctions of the entries of the $N$-site monodromy matrix of the modular XXZ magnet. We have established that each system associated with a given entry forms a complete and orthogonal system. The proof of the orthogonality was achieved by means of handlings on the level of the Gauss-Givental representation for these Eigenfunctions. The proof of the completeness was carried out on the level of the MellinBarnes representation. We stress that we have proposed a new and simple method for proving the completeness. The technique we developed is general and applicable to a wide variety of quantum integrable models solvable by the separation of variables method. As a by product of our analysis, we have proved the conjectures raised by Bytsko-Teschner on the spectrum of the $B$-operator for the lattice Sinh-Gordon model.

\section{Acknowledgments}

The authors are indebted to J.M.-Maillet for stimulating discussions. The work of S.D. is supported by the RFBR grant no. 17-01-00283a. K.K.K. acknowledges support from CNRS and ENS de Lyon. A. M. acknowledges support from the DFG grant MO 1801/1-3. S.D. would like to thank the Laboratoire de physique of ENS de Lyon for hospitality during his visit there where this work was initiated.

\section{A Main notations}

- $N$-dimensional vectors are denoted as $\boldsymbol{x}_{N}=\left(x_{1}, \ldots, x_{N}\right)$; 
- $N-1$ dimensional vectors built from an $N$-dimensional vector $\boldsymbol{x}_{N}$ with the removed $m^{\text {th }}$ coordinate are denoted as $\boldsymbol{x}_{N ;[m]}$ and read

$$
\boldsymbol{x}_{N ;[m]}=\left(x_{1}, \ldots, x_{m-1}, x_{m+1}, \ldots, x_{N}\right) .
$$

- Given an $N$-dimensional vector $\boldsymbol{x}_{N}$, we denote

$$
\overline{\boldsymbol{x}}_{N}=\sum_{a=1}^{N} x_{a}
$$

- Ratios of products of one variable functions appearing with multi-component entries are denoted using the hypergeomertic notations, e.g.

$$
f\left(\begin{array}{l}
a_{1}, \ldots, a_{n} \\
b_{1}, \ldots, b_{m}
\end{array}\right)=\frac{\prod_{k=1}^{n} f\left(a_{k}\right)}{\prod_{k=1}^{m} f\left(b_{k}\right)} .
$$

- Given indexed symbols $x_{a}, x_{b}$, we denote $x_{a b}=x_{a}-x_{b}$.

- Given $y \in \mathbb{C}, y^{*}$ stands for its complex conjugate and $y^{\star}=-y-\mathrm{i} \frac{\Omega}{2}$.

\section{B Properties of the auxiliary special functions}

\section{B.1 The quantum dilogarithm and the $D_{\alpha}$ functions}

The quantum dilogarithm $\varpi$ is a meromorphic function on $\mathbb{C}$ which admits the integral representation

$$
\varpi(\lambda)=\exp \left\{ \pm \frac{\mathrm{i} \pi}{2 \omega_{1} \omega_{2}} \cdot\left(\lambda^{2}+\frac{\omega_{1}^{2}+\omega_{2}^{2}}{12}\right)-\mathrm{i} \int_{\mathbb{R} \pm \mathrm{i} 0^{+}} \frac{\mathrm{d} t}{4 t} \frac{\mathrm{e}^{-2 \mathrm{i} \lambda t}}{\sinh \left(t \omega_{1}\right) \cdot \sinh \left(t \omega_{2}\right)}\right\},
$$

valid for $|\mathfrak{J}(\lambda)|<\Omega / 2$.

This function is self-dual and satisfies to the first-order finite difference equations

$$
\varpi\left(\lambda+\mathrm{i} \omega_{2}\right)=2 \mathrm{i} \sinh \left[\frac{\pi}{\omega_{1}}\left(\lambda+\mathrm{i} \frac{\tau}{2}\right)\right] \cdot \varpi(\lambda) \quad \text { and } \quad \varpi\left(\lambda+\mathrm{i} \omega_{1}\right)=2 \mathrm{i} \sinh \left[\frac{\pi}{\omega_{2}}\left(\lambda-\mathrm{i} \frac{\tau}{2}\right)\right] \cdot \varpi(\lambda) .
$$

From there one entails that

$$
\begin{aligned}
\varpi\left(\lambda-\mathrm{i} \frac{\Omega}{2}+\mathrm{i} \ell \omega_{1}+\mathrm{i} k \omega_{2}\right)=(-1)^{k \ell}(-2 \mathrm{i})^{\ell+k} \cdot \prod_{p=0}^{k-1} \sinh \left[\frac{\pi}{\omega_{1}}\left(\lambda+\mathrm{i} p \omega_{2}\right)\right] \\
\times \prod_{p=0}^{\ell-1} \sinh \left[\frac{\pi}{\omega_{2}}\left(\lambda+\mathrm{i} p \omega_{1}\right)\right] \cdot \varpi\left(\lambda-\mathrm{i} \frac{\Omega}{2}\right)
\end{aligned}
$$


and symmetrically,

$$
\begin{aligned}
\varpi\left(\lambda-\mathrm{i} \frac{\Omega}{2}-\mathrm{i} \ell \omega_{1}-\mathrm{i} k \omega_{2}\right)=(-1)^{k \ell}\left(\frac{\mathrm{i}}{2}\right)^{\ell+k} \cdot \prod_{p=1}^{k}\{\sinh & {\left.\left[\frac{\pi}{\omega_{1}}\left(\lambda-\mathrm{i} p \omega_{2}\right)\right]\right\}^{-1} } \\
& \times \prod_{p=1}^{\ell}\left\{\sinh \left[\frac{\pi}{\omega_{2}}\left(\lambda-\mathrm{i} p \omega_{1}\right)\right]\right\}^{-1} \cdot \varpi\left(\lambda-\mathrm{i} \frac{\Omega}{2}\right) .
\end{aligned}
$$

The quantum dilogarithm has only simple poles and zeroes. These are located at

$$
\varpi(x)=0 \quad \text { iff } \quad x \in \mathrm{i} \frac{\Omega}{2}+\mathrm{i} \mathbb{N} \omega_{1}+\mathrm{i} \mathbb{N} \omega_{2} \quad \text { and } \quad \varpi^{-1}(x)=0 \quad \text { iff } \quad x \in-\mathrm{i} \frac{\Omega}{2}-\mathrm{i} \mathbb{N} \omega_{1}-\mathrm{i} \mathbb{N} \omega_{2} .
$$

$\varpi$ satisfies to the inversion identity $\varpi(\lambda) \varpi(-\lambda)=1$ and $\left(\varpi\left(\lambda^{*}\right)\right)^{*}=\varpi^{-1}(\lambda)$. One can also establish that

$$
\operatorname{Res}\left(\varpi\left(\lambda-\mathrm{i} \frac{\Omega}{2}\right) \cdot \mathrm{d} \lambda, \lambda=0\right)=\frac{\mathrm{i}}{2 \pi} \sqrt{\omega_{1} \omega_{2}} \quad \text { and } \quad \varpi\left(\frac{\mathrm{i}}{2} \tau\right)=\sqrt{\frac{\omega_{2}}{\omega_{1}}} .
$$

The above entails that, for $(k, \ell) \in \mathbb{N}^{2}$,

$$
\operatorname{Res}\left(\varpi\left(\lambda-\mathrm{i} \frac{\Omega}{2}\right) \cdot \mathrm{d} \lambda, \lambda=-\mathrm{i} \ell \omega_{1}-\mathrm{i} k \omega_{2}\right)=\frac{\mathrm{i}}{2 \pi} \sqrt{\omega_{1} \omega_{2}}(-1)^{k \ell}\left(\frac{1}{2 \mathrm{i}}\right)^{\ell+k} \cdot\left\{\prod_{p=1}^{k} \sinh \left[\mathrm{i} p \pi \frac{\omega_{2}}{\omega_{1}}\right] \cdot \prod_{p=1}^{\ell} \sinh \left[\mathrm{i} p \pi \frac{\omega_{1}}{\omega_{2}}\right]\right\}^{-1} .
$$

The function $D_{\alpha}$ is defined by the below ratio of dilogarithms

$$
D_{\alpha}(\lambda)=\frac{\varpi(\lambda+\alpha)}{\varpi(\lambda-\alpha)} \quad \text { so that } \quad D_{\alpha}(\lambda)=\left(D_{-\alpha^{*}}\left(\lambda^{*}\right)\right)^{*} .
$$

The function $D_{\alpha}$ is a meromorphic function on $\mathbb{C}$ that admits a holomorphic determination of the logarithm on

$$
\mathcal{S}_{\frac{\Omega}{2}-|\mathfrak{I}(\alpha)|}(\mathbb{R})=\left\{\lambda \in \mathbb{C}:|\mathfrak{J}(\lambda)|<\frac{1}{2}\left(\omega_{1}+\omega_{2}\right)-|\mathfrak{I}(\alpha)|\right\}
$$

given by

$$
\ln D_{\alpha}(\lambda)=\mp \frac{2 \mathrm{i} \pi}{\omega_{1} \omega_{2}} \alpha \lambda+\mathrm{i} \int_{\mathbb{R} \pm 0^{+}} \frac{\mathrm{d} t}{2 t} \frac{\mathrm{e}^{2 \mathrm{i} \lambda t} \sin (2 \alpha t)}{\sinh \left(t \omega_{1}\right) \cdot \sinh \left(t \omega_{2}\right)} .
$$

The function $D_{\alpha}(\lambda)$ satisfies to the properties

- $D_{\alpha}(\lambda)$ is self-dual, namely invariant under the exchange $\omega_{1} \leftrightarrow \omega_{2}$;

- for $\omega_{2}>\omega_{1}$ it admits the asymptotic behaviours

$$
D_{\alpha}(\lambda)=\mathrm{e}^{\mp \frac{2 i \pi}{\omega_{1} \omega_{2}} \cdot \lambda \alpha} \cdot\left(1+\mathrm{O}\left(\mathrm{e}^{\mp \frac{2 \pi}{\omega_{2}} \lambda} \sinh \left[\frac{2 \pi}{\omega_{2}} \alpha\right]\right)\right) \text { when } \lambda \rightarrow \infty, \quad|\arg ( \pm \lambda)|<\frac{\pi}{2} ;
$$

- $D_{\alpha}(\lambda)$ satisfies to the difference equation

$$
\frac{D_{\alpha}\left(\lambda+\mathrm{i} \omega_{2}\right)}{D_{\alpha}(\lambda)}=\frac{\cosh \left[\frac{\pi}{\omega_{1}}\left(\lambda+\mathrm{i} \frac{\omega_{2}}{2}+\alpha\right)\right]}{\cosh \left[\frac{\pi}{\omega_{1}}\left(\lambda+\mathrm{i} \frac{\omega_{2}}{2}-\alpha\right)\right]}
$$

as well as its dual $\omega_{1} \leftrightarrow \omega_{2}$; 
- $D_{\alpha}(\lambda)$ enjoys the transmutation properties

$$
D_{\alpha}\left(\lambda \mp \mathrm{i} \frac{\omega_{2}}{2}\right)=\frac{D_{\alpha-\mathrm{i} \frac{\omega_{2}}{2}}(\lambda)}{2 \cosh \left[\frac{\pi}{\omega_{1}}(\lambda \pm \alpha)\right]} ;
$$

- $D_{\alpha}(\lambda)$ has simple zeroes at

$$
\pm\left\{-\alpha+\mathrm{i} \frac{\Omega}{2}+\mathrm{i} n \omega_{1}+\mathrm{i} m \omega_{2}:(n, m) \in \mathbb{N}^{2}\right\} ;
$$

- $D_{\alpha}(\lambda)$ has simple poles at

$$
\pm\left\{\alpha+\mathrm{i} \frac{\Omega}{2}+\mathrm{i} n \omega_{1}+\mathrm{i} m \omega_{2}:(n, m) \in \mathbb{N}^{2}\right\} .
$$

\section{B.2 Integral identities}

The function $D_{\alpha}$ admits the Fourier transform

$$
\mathcal{F}\left[D_{\alpha}\right](t)=\sqrt{\omega_{1} \omega_{2}} \cdot \mathcal{A}(\alpha) \cdot D_{\alpha^{\star}}\left(\frac{\omega_{1} \omega_{2}}{2 \pi} t\right)
$$

with

$$
\mathcal{F}[f](t)=\int_{\mathbb{R}} \mathrm{e}^{i t x} f(x) \cdot \mathrm{d} x \quad \text { for } \quad f \in L^{1}(\mathbb{R}, \mathrm{d} x) .
$$

Here we remind that

$$
\mathcal{A}(\alpha)=\varpi\left(2 \alpha+\mathrm{i} \frac{\Omega}{2}\right) \quad \text { and } \quad \alpha^{\star}=-\alpha-\mathrm{i} \frac{\Omega}{2} .
$$

(B.16) enatils that

$$
\lim _{\alpha \rightarrow 0}\left\{\mathcal{A}(\alpha) D_{\alpha^{\star}}(t)\right\}=\delta(t) .
$$

The $D_{\alpha}$ functions satisfy to the three term integral identity [14]

$$
\begin{aligned}
\int_{\mathbb{R}} D_{\alpha}\left(\omega_{1} \omega_{2}(x-u)\right) \cdot D_{\beta}\left(\omega_{1} \omega_{2}(x-v)\right) \cdot D_{\gamma}\left(\omega_{1} \omega_{2}(x-w)\right) \cdot \mathrm{d} x \\
=\frac{\mathcal{A}(\alpha, \beta, \gamma)}{\sqrt{\omega_{1} \omega_{2}}} D_{\alpha^{\star}}\left(\omega_{1} \omega_{2}(w-v)\right) \cdot D_{\beta^{\star}}\left(\omega_{1} \omega_{2}(u-w)\right) \cdot D_{\gamma^{\star}}\left(\omega_{1} \omega_{2}(u-v)\right)
\end{aligned}
$$

provided that $\alpha+\beta+\gamma=-\mathrm{i} \Omega$ and [3]

$$
\begin{aligned}
& \int_{\mathbb{R}} D_{\alpha}\left(\omega_{1} \omega_{2}(x-u)\right) \cdot D_{\beta}\left(\omega_{1} \omega_{2}(x-v)\right) \cdot D_{\gamma}\left(\omega_{1} \omega_{2}(x-w)\right) \cdot D_{\delta}\left(\omega_{1} \omega_{2}(x-z)\right) \cdot \mathrm{d} x \\
& =\mathcal{A}(\alpha, \beta, \gamma, \delta) \cdot D_{\alpha+\beta+\mathrm{i} \frac{\Omega}{2}}\left(\begin{array}{c}
\omega_{1} \omega_{2}(u-v) \\
\omega_{1} \omega_{2}(w-z)
\end{array}\right) \\
& \quad \times \int_{\mathbb{R}} D_{\alpha^{\star}}\left(\omega_{1} \omega_{2}(x-v)\right) \cdot D_{\beta^{\star}}\left(\omega_{1} \omega_{2}(x-u)\right) \cdot D_{\gamma^{\star}}\left(\omega_{1} \omega_{2}(x-z)\right) \cdot D_{\delta^{\star}}\left(\omega_{1} \omega_{2}(x-w)\right) \cdot \mathrm{d} x
\end{aligned}
$$


provided that $\alpha+\beta+\gamma+\delta=-\mathrm{i} \Omega$.

Sending one of the integration variables to infinity provides one with the auxiliary identities

$$
\int_{\mathbb{R}} D_{\alpha}\left(\omega_{1} \omega_{2}(x-u)\right) \cdot \mathrm{e}^{ \pm 2 \mathrm{i} \pi \beta x} \cdot D_{\gamma}\left(\omega_{1} \omega_{2}(x-w)\right) \cdot \mathrm{d} x=\frac{\mathcal{A}(\alpha, \beta, \gamma)}{\sqrt{\omega_{1} \omega_{2}}} \cdot \mathrm{e}^{ \pm 2 \mathrm{i} \pi\left(\alpha^{\star} w+\gamma^{\star} u\right)} D_{\beta^{\star}}\left(\omega_{1} \omega_{2}(u-w)\right)
$$

provided that that $\alpha+\beta+\gamma=-\mathrm{i} \Omega$ and

$$
\begin{aligned}
& \int_{\mathbb{R}} D_{\alpha}\left(\omega_{1} \omega_{2}(x-u)\right) \cdot D_{\beta}\left(\omega_{1} \omega_{2}(x-v)\right) \cdot D_{\gamma}\left(\omega_{1} \omega_{2}(x-w)\right) \cdot \mathrm{e}^{ \pm 2 \mathrm{i} \pi \delta x} \cdot \mathrm{d} x \\
&=\mathcal{A}(\alpha, \beta, \gamma, \delta) \mathrm{e}^{ \pm 2 \mathrm{i} \pi\left(\alpha+\beta+\mathrm{i} \frac{\Omega}{2}\right){ }^{w}} D_{\alpha+\beta+\mathrm{i} \frac{\Omega}{2}}(u-v) \\
& \quad \times \int_{\mathbb{R}} D_{\alpha^{\star}}\left(\omega_{1} \omega_{2}(x-v)\right) \cdot D_{\beta^{\star}}\left(\omega_{1} \omega_{2}(x-u)\right) \cdot D_{\delta^{\star}}\left(\omega_{1} \omega_{2}(x-w)\right) \cdot \mathrm{e}^{ \pm 2 \mathrm{i} \pi \gamma^{\star} x} \cdot \mathrm{d} x
\end{aligned}
$$

provided that $\alpha+\beta+\gamma+\delta=-\mathrm{i} \Omega$.

The three term integral relation can be recast in an operator form as

$$
D_{u}(\mathrm{p}) \cdot D_{u+v}\left(\omega_{1} \omega_{2} \mathrm{x}\right) \cdot D_{v}(\mathrm{p})=D_{v}\left(\omega_{1} \omega_{2} \mathrm{x}\right) \cdot D_{u+v}(\mathrm{p}) \cdot D_{u}\left(\omega_{1} \omega_{2} \mathrm{x}\right)
$$

whereas its degenerate form can be recast as

$$
D_{\alpha}(\mathrm{p}) \cdot \mathrm{e}^{ \pm 2 \mathrm{i} \pi \beta \mathrm{x}} \cdot D_{\gamma}(\mathrm{p})=\mathrm{e}^{ \pm 2 \mathrm{i} \pi \gamma \mathrm{x}} \cdot D_{\beta}(\mathrm{p}) \cdot \mathrm{e}^{ \pm 2 \mathrm{i} \pi \alpha \mathrm{x}}
$$

where $\alpha, \beta, \gamma$ fulfill the constraint $\beta=\alpha+\gamma$.

Let $y_{ \pm}, t_{ \pm}$be parameters as in (3.1). Then, the integral identity involving four $D$ functions can be recast in the operator form as

$$
\begin{aligned}
D_{y_{-}}\left(\mathrm{p}_{2}\right) & \cdot D_{y_{+}}\left(\omega_{1} \omega_{2} \mathrm{x}_{23}\right) \cdot D_{t_{-}}\left(\omega_{1} \omega_{2} \mathrm{x}_{12}\right) \cdot D_{t_{+}}\left(\mathrm{p}_{2}\right) \\
& =D_{y_{-}^{\star}+t_{-}+\mathrm{i} \frac{\Omega}{2}}\left(\omega_{1} \omega_{2} \mathrm{x}_{12}\right) \cdot D_{t_{-}}\left(\mathrm{p}_{2}\right) \cdot D_{t_{+}}\left(\omega_{1} \omega_{2} \mathrm{x}_{23}\right) \cdot D_{y_{-}}\left(\omega_{1} \omega_{2} \mathrm{x}_{12}\right) \cdot D_{y_{+}}\left(\mathrm{p}_{2}\right) \cdot D_{y_{-}^{\star}+t_{-}+\mathrm{i} \frac{\Omega}{2}}^{-1}\left(\omega_{1} \omega_{2} \mathrm{x}_{32}\right) .
\end{aligned}
$$

Likewise, its exponential degenerate form can be recast as

$$
\begin{aligned}
\mathrm{e}^{2 \mathrm{i} \pi y_{-} \mathrm{x}_{1}} \cdot D_{y_{-}}\left(\mathrm{p}_{1}\right) \cdot D_{y_{+}}\left(\omega_{1} \omega_{2} \mathrm{x}_{12}\right) \cdot \mathrm{e}^{2 \mathrm{i} \pi t_{-} \mathrm{x}_{1}} \cdot D_{t_{+}}\left(\mathrm{p}_{1}\right) \\
\quad=\mathrm{e}^{2 \mathrm{i} \pi t_{-} \mathrm{x}_{1}} \cdot D_{t_{-}}\left(\mathrm{p}_{1}\right) \cdot D_{t_{+}}\left(\omega_{1} \omega_{2} \mathrm{x}_{12}\right) \cdot \mathrm{e}^{2 \mathrm{i} \pi y_{-} \mathrm{x}_{1}} \cdot D_{y_{+}}\left(\mathrm{p}_{1}\right) \cdot D_{y_{-}+t_{-}+\mathrm{i} \frac{\Omega}{2}}^{-1}\left(\omega_{1} \omega_{2} \mathrm{x}_{21}\right) .
\end{aligned}
$$

Finally, the exponential degenerate form of the four D function integral (B.23) can be also recast as

$$
\begin{aligned}
\mathcal{A}\left(t_{+}\right) D_{y_{-}}\left(\mathrm{p}_{2}\right) \cdot D_{y_{+}}\left(\omega_{1} \omega_{2} \mathrm{x}_{23}\right) \cdot \mathrm{e}^{ \pm 2 \mathrm{i} \pi\left(t_{+}^{\star} \mathrm{x}_{2}+y_{+}^{\star} \mathrm{x}_{3}\right)} \cdot D_{t_{-}}\left(\omega_{1} \omega_{2} \mathrm{x}_{12}\right) \\
=\mathcal{A}\left(y_{+}\right) D_{y_{-}^{\star}+t_{-}+\mathrm{i} \frac{\Omega}{2}}\left(\omega_{1} \omega_{2} \mathrm{x}_{12}\right) D_{t_{-}}\left(\mathrm{p}_{2}\right) \cdot D_{t_{+}}\left(\omega_{1} \omega_{2} \mathrm{x}_{23}\right) \cdot \mathrm{e}^{ \pm 2 \mathrm{i} \pi\left(y_{+}^{\star} \mathrm{x}_{2}+t_{+}^{\star} \mathrm{x}_{3}\right)} \cdot D_{y_{-}}\left(\omega_{1} \omega_{2} \mathrm{x}_{12}\right) .
\end{aligned}
$$




\section{References}

[1] O. Babelon and K.K. Kozlowski and V. Pasquier, "Baxter operator and Baxter Equation for q-Toda and Toda 2 chains.", Ludwig Faddeev Memorial Volume", World Scientific. (2018)

[2] A.G. Bytsko and J. Teschner, "R-operator, co-product and Haar-measure for the modular double of $U_{q}(\mathfrak{s l}(2, R))$.", Commun.Math.Phys. 240 (2003), 171-196.

[3] _ _ "Quantization of models with non-compact quantum group symmetry. Modular XXZ magnet and lattice sinh-Gordon model.", J. Phys. A 39 (2006), 12927-12982.

[4] D. Chicherin and S. Derkachov, "The R-operator for a modular double.", J.Phys. A: Math. and Theor. 47 (2005), 115203.

[5] D. Chicherin, S. Derkachov, D. Karakhanyan, and R. Kirschner., "Baxter operators with deformed symmetry.", Nucl.Phys. B 868 (2013), 652-683.

[6] S.E. Derkachov, "Baxter's Q-operator for the homogeneous XXX spin chain", J. Phys. A 32 (1999), 5299_ 5316.

[7] S.E. Derkachov, G.P. Korchemsky, and A.N. Manashov, "Noncompact Heisenberg spin magnets from highenergy QCD: I. Baxter Q-operator and separation of variables.", Nucl. Phys. B 617 (2001), 375-440.

[8] _ _ "Separation of variables for the quantum SL(2,R) spin chain. ", JHEP 0307 (2003), 047.

[9] S.E. Derkachov and A.N. Manashov, "Iterative construction of eigenfunctions of the monodromy matrix for SL(2,C) magnet.", J.Phys. A: Math. gen. 47 (2014), 305204.

[10] M. Gaudin and V. Pasquier, "The periodic Toda chain and a matrix generalization of the Bessel function recursion relations.", J. Phys. A 25 (1992), 5243-5252.

[11] A. Gerasimov, S. Kharchev, and D. Lebedev, "Representation theory and quantum inverse scattering method: the open Toda chain and the hyperbolic Sutherland model.", Int. Math. Res. Notices 2004.

[12] A. Givental, "Stationary phase integrals, quantum Toda lattices, flag manifolds and the mirror conjecture.", AMS Trans. (2) 180 (1997), 103-115.

[13] M.C. Gutzwiller, "The quantum mechanical Toda lattice.", Ann. Phys. 124 (1980), 347-387.

[14] R. Kashaev, "The Quantum Dilogarithm and Dehn Twists in Quantum TeichmÃIJller Theory.", Integrable Structures of Exactly Solvable Two-Dimensional Models of Quantum Field Theory. NATO Science Series (Series II: Mathematics, Physics and Chemistry), vol 35. Springer, Dordrecht. Edts: Pakuliak S., von Gehlen G. (2001), 211-221.

[15] S. Kharchev and D. Lebedev, "Eigenfunctions of $G L(N, \mathbb{R})$ Toda chain: The Mellin-Barnes representation.", JETP Lett. 71 (2000), 235-238.

[16] _ "Integral representations for the eigenfunctions of quantum open and periodic Toda chains from QISM formalism.", J.Phys.A 34 (2001), 2247-2258.

[17] S. Kharchev, D. Lebedev, and M. Semenov-Tian-Shansky, "Unitary representations of $U_{q}(\mathfrak{s l}(2, \mathbb{R}))$, the modular double and the multiparticle q-deformed Toda chains.", Comm. Math. Phys. 225 (2002), 573-609. 
[18] K.K. Kozlowski, "Aspects of the inverse problem for the Toda chain.", J. Math. Phys. 54 (2013), 121902.

[19] _ , "Unitarity of the SoV transform for the Toda chain.", Comm. Math. Phys. 334 (2015), no. 1, 223273.

[20] G. Niccoli and V. Terras, "The eight-vertex model with quasi-periodic boundary conditions.", J. Phys. A: Math. Theor. 49 (2016), 044001, 37pp.

[21] G. Niccoli and J. Teschner, "The Sine-Gordon model revisited I.", J.Stat.Mech. 1009 (2010), P09014.

[22] G. Schrader and A. Shapiro, "On b-Whittaker functions.", math-ph:1806.00747.

[23] A.V. Silantyev, "Transition function for the Toda chain.", Theor. Math. Phys. 150 (2007), 315-331.

[24] E.K. Sklyanin, "The quantum Toda chain.", Lect. Notes in Phys. 226 (1985), 196-233.

[25] __ , "Functional Bethe Ansatz.", Integrable and Superintegrable Theories (Ed. Kupershmidt, B.), Singapore: World Scientific (1990), 8ÃćÂĂÂŞ33.

[26] __ "Quantum inverse scattering method. Selected topics.", in "Quantum Group and Quantum Integrable Systems" (Nankai Lectures in Mathematical Physics), ed. by Mo-Lin Ge, Singapore: World Scientific (1992), 63-97.

[27] N.R. Wallach, "Real reductive groups II.", Pure and applied mathematics, vol. 132-II, Academic Press, inc., 1992. 